\title{
The Propagation Mechanism of High Speed Turbulent Deflagrations.
}

\author{
by \\ Jenny C. Chao \\ Department of Mechanical Engineering \\ McGill University \\ Montreal, Quebec, Canada
}

\begin{abstract}
A thesis submitted to the
Faculty of Graduate Studies and Research

in partial fulfillment of the requirements for the degree of Master's of Engineering.
\end{abstract}

(C) Jenny C. Chao

January 2002 
National Librany

of Canada

Acquisitions and Bibliographic Services

305 Wollingion Strept Orime ON KIA ONA Cenece
Bibliotheque nationale

du Canada

Acquisitions ef

services bibliographiques

395. nu Wellington

Oriam ON KIA ONA

Canede
The author has granted a nonexclusive licence allowing the National Library of Canada to reproduce, loan, distribute or sell copies of this thesis in microform, paper or electronic formats.

The author retains ownership of the copyright in this thesis. Neither the thesis nor substantial extracts from it may be printed or otherwise reproduced without the author's permission.
L'auteur a accordé une licence non exchusive permettant à la Bibliothèque nationale du Canada de reproduire, prêter, distribuer ou vendre des copies de cette these sous la forme de microfiche/film, de reproduction sur papier ou sur format électronique.

L'auteur conserve la propriété du droit d'auteur qui protège cette thèse. Ni la thèse ni des extraits substantiels de celle-ci ne doivent être imprimés ou autrement reproduits sans son autorisation. 


\section{Abstract}

The propagation regimes of combustion waves in a $30 \mathrm{~cm}$ by $30 \mathrm{~cm}$ square cross-sectioned tube with an obstacle array of staggered vertical cylindrical rods (with $\mathrm{BR}=0.41$ and $\mathrm{BR}=0.19)$ are investigated. Mixtures of hydrogen, ethylene, propane, and methane with air at ambient conditions over a range of equivalence ratios are used. In contrast to the previous results obtained in circular cross-sectioned tubes, it is found that only the quasi-detonation regime and the slow turbulent deflagration regimes are observed for ethylene-air and for propane-air. The transition from the quasi-detonation regime to the slow turbulent deflagration regime occurs at $D / \lambda \approx 1$ (where $D$ is the tube "diameter" and $\lambda$ is the detonation cell size). When $D / \lambda>>1$, the quasi-detonation velocities that are observed are similar to those in unobstructed smooth tubes. For hydrogen-air mixtures, it is found that there is a gradual transition from the quasi-detonation regime to the high speed turbulent deflagration regime. The high speed turbulent deflagration regime is also observed for methane-air mixtures near stoichiometric composition. This regime was previously interpreted as the "choking" regime in circular tubes with orifice plate obstacles. Presently, it is proposed that the propagation mechanism of these high speed turbulent deflagrations is similar to that of Chapman-Jouguet detonations and quasi-detonations. As well, it is observed that there exists unstable flame propagation at the lean limit where $D / \lambda \approx 1$. The local velocity fluctuates significantly about an averaged velocity for hydrogen-air, ethylene-air, and propane-air mixtures. Unstable flame propagation is also observed for the entire range of high speed turbulent deflagrations in methane-air mixtures. It is proposed that these fluctuations are due to quenching of the combustion front due to turbulent mixing. Quenched pockets of unburned reactants are swept downstream, and the subsequent explosion serves to overdrive the combustion front. The present study indicates that the dependence on the propagation mechanisms on obstacle geometry can be exploited to elucidate the different complex mechanisms of supersonic combustion waves. 


\section{Résumé}

Dans cette thèse, les différents régimes de propagation des ondes de combustion ont été étudiés dans un tube carré $(30 \mathrm{~cm}$ par $30 \mathrm{~cm})$ dans lequel une série d'obstructions cylindriques a été introduite (perpendiculairement à l'écoulement) avec un rapport de blocage de 0,41 et 0,19 . Des combustibles d'hydrogène, d'éthylène, de propane, et de méthane mélangés en différentes proportions avec de l'air ont été testés. Contrairement aux études ultérieures menées dans des tubes ronds munis obstacles annulaires, seulement les régimes de "quasi-détonations» et de déflagrations turbulentes lentes ont été observées pour les mélanges d'éthylène et de propane. La transition entre les deux régimes se situe à la limite $\mathrm{D} / \lambda \approx 1$ (où $\mathrm{D}$ est le diamètre du tube et $\lambda$ est la dimension caractéristique des mailles). Pour des conditions telles que $\mathrm{D} / \lambda \approx 1$, les célérités des "quasi-détonations» se rapprochent de celles expérimentées dans des tubes nonobstrués. Des déflagrations de grande célérité ont été observées dans les mélanges d'hydrogène près de la limite $\mathrm{D} / \lambda \approx 1$ et dans les mélanges de méthane-air approchant la composition stoichiométrique. Lorsque étudiées dans les tubes à obstacles annulaires, ces ondes étaient auparavant interprétées comme un écoulement forcé. On propose que le mécanisme de propagation de ces déflagrations supersoniques soit très similaire au mécanisme des détonations Chapman-Jouguet et des « quasi-détonations ». Les mélanges sous stoichiometriques d'hydrogène-air, d'éthylène-air et de propane-air ont démontré de larges fluctuations dans un régime instable de combustion situé sur la limite $D / \lambda \approx 1$. Ces larges fluctuations caractérisent également toutes les stoichiométries de méthane-air pour lesquelles des ondes de combustion supersoniques sont observées. On propose que ces fluctuations et instabilités soient dues à l'extinction locale du front de combustion généré par la turbulence induite par les obstacles. Suite à l'extinction partielle, des poches de gaz non-brûlé se retrouvent derrière le front, réagissent suite à un délai et créent de fortes ondes de pression qui altèrent le front de l'onde globale. Cette étude a permis d'étudier les différents mécanismes de propagation des ondes de combustion supersoniques et de déterminer les conditions nécessaires à leur existence. 


\section{Acknowledgements}

And a man said, Speak to us of Self-Knowledge.

And he answered, saying:

Your hearts know in silence the secrets of the days and the nights.

But your ears thirst for the sound of your heart's knowledge.

You would know in words that which you have always known in thought... truth."

Say not, "I have found the truth," but rather, "I have found a

From The Prophet by Kahlil Gibran

First and foremost, I would like to thank my supervisor, Prof. John H.S. Lee, for his faith in my potential and for his guidance throughout my search of "Self-Knowledge."

I am grateful to every former and present member of the Shock Wave Physics Group. Although I would like to distinguish each of my colleagues' contribution to my research or to my self-preservation, I would like to thank, in particular, Massimiliano Kolbe, Matei Radulescu, Massimiliano Romano, Vincent Tanguay, Pierre Pinard, and Della Maharajh. The insight and the assistance with the last few experiments received from Dr. Ramamurthi is also greatly appreciated. I would also like acknowledge the technicians in the Mechanical Laboratory and in the Measurement Laboratory for their invaluable help.

I am also very grateful for the support from all of my friends and my family; in particular, I would like to thank John Alton for his friendship, his patience, and his understanding.

Lastly and most importantly, I would like to thank both my loving parents for everything. I can not put into words all that they have done for me. 


\section{Table of contents}

Abstract __ i

Résumé __ii

Acknowledgements __ iii

List of Figures _ vi

List of Tables _

Chapter 1 Introduction ___ 1

1.1 Historical background __ 1

1.2 Motivation _ـ 2

1.3 Previous work _ 2

1.4 Limitation of previous work __ 3

1.5 Classification of steady state combustion waves __ 4

1.6 Present work _ـ 5

Chapter 2 Experimental details __ 6

2.1 Experimental apparatus ___ 6

2.2 Mixture selection and preparation ___ _ 7

2.3 Diagnostics __ 7

Chapter 3 Results and discussion _ 9

3.1 General observations __ 9

3.2 Steady state regimes ___ 10

3.2.1 Quasi-detonation regime 10

3.2.2 High speed turbulent deflagration regime

3.2.3 Transition to the slow turbulent deflagrations regime ___ 14

3.2.4 Absence of the "choking" regime _ 16

3.3 Unstable regimes at the transition limit _ 16

3.3.1 Absence of unstable regimes at rich transition limit

3.3.2 Effect of blockage ratio on unstable regime _ـ 20

3.4 Methane-air__ 21

3.4.1 Sensitization of methane-air with 5\% ethane ___ 21

3.4.2 The importance of transverse shocks _ 22 
Chapter 4 Concluding remarks 24

4.1 Future work 25

References 27

Figures 30 


\section{List of Figures}

Fig. 1: $\quad$ Top view of tube-obstacle assembly.

Fig. 2: $\quad$ Photograph of tube and obstacle array.

Fig. 3: $\quad$ Schematic of experimental set-up.

Fig. 4: Typical oscilloscope signals from ionization probes for stoichiometric propane-air $(\phi=1.0, \mathrm{BR}=0.41)$.

Fig. 5: $\quad$ Typical pressure profile of a high speed turbulent deflagration for stoichiometric methane-air $(\phi=1.0, \mathrm{BR}=0.41)$.

Fig. 6: Typical pressure profile of a quasi-detonation for stoichiometric propane-air $(\phi=1.0, \mathrm{BR}=0.41)$.

Fig. 7: $\quad$ Typical pressure profile of a slow turbulent deflagration for very lean propane-air $(\phi=0.55, \mathrm{BR}=0.41)$.

Fig. 8: $\quad$ Flame trajectory for stoichiometric propane-air $(\phi=1.0, \mathrm{BR}=0.41)$.

Fig. 9: Local velocity variation with distance along tube for stoichiometric propane-air $(\phi=1.0, \mathrm{BR}=0.41)$.

Fig. 10: Local velocity variation with distance along tube for rich propane-air $(\phi=1.4, \mathrm{BR}=0.41)$ with and without driver section.

Fig. 11: a) Local velocity variation with distance along tube for stoichiometric hydrogen-air $(\phi=1.0, \mathrm{BR}=0.41)$. b) Corresponding velocity histogram.

Fig. 12: a) Local velocity variation with distance along tube for hydrogen-air near lean limit of quasi-detonation regime $(\phi=0.6, \mathrm{BR}=0.41)$.

b) Corresponding velocity histogram.

Fig. 13: a) Local velocity variation with distance along tube for hydrogen-air near rich limit of quasi-detonation regime $(\phi=3.1, \mathrm{BR}=0.41)$.

b) Corresponding velocity histogram. 
Fig. 14: a) Local velocity variation with distance along tube for stoichiometric ethylene-air $(\phi=1.0, \mathrm{BR}=0.41)$. b) Corresponding velocity histogram.

Fig. 15: a) Local velocity variation with distance along tube for ethylene-air near lean limit of quasi-detonation regime $(\phi=0.7, \mathrm{BR}=0.41)$.

b) Corresponding velocity histogram.

Fig. 16: a) Local velocity variation with distance along tube for ethylene-air near rich limit of quasi-detonation regime $(\phi=2.2, \mathrm{BR}=0.41)$.

b) Corresponding velocity histogram.

Fig. 17: a) Local velocity variation with distance along tube for stoichiometric propane-air $(\phi=1.0, \mathrm{BR}=0.41)$. b) Corresponding velocity histogram.

Fig. 18: a) Local velocity variation with distance along tube for propane-air near lean limit of quasi-detonation regime $(\phi=0.8, \mathrm{BR}=0.41)$.

b) Corresponding velocity histogram.

Fig. 19: a) Local velocity variation with distance along tube for propane-air near rich limit of quasi-detonation regime $(\phi=1.3, \mathrm{BR}=0.41)$.

b) Corresponding velocity histogram.

Fig. 20: Variation of velocity with $\%$ hydrogen in hydrogen-air.

Fig. 21: Variation of velocity with equivalence ratio for ethylene-air.

Fig. 22: Variation of velocity with equivalence ratio for propane-air.

Fig. 23: a) Local velocity variation with distance along tube for rich hydrogen-air $(\phi=5.55, \mathrm{BR}=0.41)$ in high speed turbulent deflagration regime.

b) Corresponding velocity histogram.

Fig. 24: Variation of post-shock temperature of a C-J detonation with equivalence ratio for hydrogen-air.

Fig. 25: a) Computer simulation of flame propagation in "choking" regime. b) High speed Schlieren photograph of flame propagation in "choking" regime. 
Fig. 26: a) Local velocity variation with distance along tube for lean hydrogen-air $(\phi=0.52, \mathrm{BR}=0.41)$ at lean transition limit. $\mathrm{b})$ Corresponding velocity histogram.

Fig. 27: a) Local velocity variation with distance along tube for lean ethylene-air $(\phi=0.6, \mathrm{BR}=0.41)$ at lean transition limit. $\mathrm{b})$ Corresponding velocity histogram.

Fig. 28: a) Local velocity variation with distance along tube for lean propane-air $(\phi=0.7, \mathrm{BR}=0.41)$ at lean transition limit. $\mathrm{b})$ Corresponding velocity histogram.

Fig. 29: Streak photographs of a) quasi-detonation, b) unstable flame propagation at transition limit between quasi-detonation regime and "choking" regime, and c) stable flame propagation at transition limit with $75 \%$ argon dilution (all in stoichiometric acetylene-oxygen).

Fig. 30: Local velocity variation with distance along tube for lean ethylene-air $(\phi=0.6)$ using different blockage ratio at lean transition limit.

Fig. 31: Local velocity variation with distance along tube for lean propane-air $(\phi=0.7)$ using different blockage ratio at lean transition limit.

Fig. 32: a) Local velocity variation with distance along tube for stoichiometric methane-air $(\phi=1.0, \mathrm{BR}=0.41)$. b) Corresponding velocity histogram.

Fig. 33: Variation of velocity with equivalence ratio for methane-air.

Fig. 34: Local velocity variation with distance along tube for stoichiometric methane-air $(\phi=1.0, \mathrm{BR}=0.19)$.

Fig. 35: Local velocity variation with distance along tube for stoichiometric methane-air with $5 \%$ ethane $(\phi=1.0, \mathrm{BR}=0.41)$.

Fig. 36: Local velocity variation with distance along tube for stoichiometric methane-air $(\phi=1.0, \mathrm{BR}=0.41)$ with and without acoustically absorbing material. 
Fig. 37: Open shutter photograph of shock wave $\left(M_{s} \approx 1.3\right)$ propagating through dense obstacle field.

Fig. 38: Local velocity variation with distance along tube for stoichiometric methane-air $(\phi=1.0, \mathrm{BR}=0.41)$ into an empty tube. 


\section{List of Tables}

Table 1: Correlation of different physical length scales with $\lambda$ at transition limits.

Table 2: $\quad$ Quenching diameter and MESG for different stoichiometric fuel-air mixtures.

Table 3: Concentration of $\mathrm{H}$ radicals at transition limits for different fuel-air mixtures. 


\section{Chapter 1 Introduction}

\subsection{Historical background}

It has been well-established that a propagating flame - or a deflagration - in a gaseous fuel-oxidizer mixture can accelerate to very high steady state velocities in very rough-walled or obstacle-filled tubes. This has been found to be possible even for insensitive mixtures. The pioneering experiments of Chapman and Wheeler have demonstrated that an otherwise laminar methane-air flame traveling at $14 \mathrm{~cm} / \mathrm{s}$ in a smooth tube can be accelerated to a steady velocity of $420 \mathrm{~m} / \mathrm{s}$ by placing annular rings (or orifice plates) in its path. ${ }^{1}$ Under appropriate conditions, a deflagration can transition to a detonation, which is commonly referred to as DDT (deflagration to detonation transition). By roughening the confining tube, the distance traveled by the combustion wave before DDT can be rendered much shorter. This was first observed by Laffitte ${ }^{2}$ who found that upon lining a tube with sand, the run-up distance to detonation would decrease twofold from $84 \mathrm{~cm}$ to $45 \mathrm{~cm}$. Almost two decades later, Shchelkin's ${ }^{3}$ observations of DDT in a tube fitted with a helical wire spiral corroborated those made by Laffitte. In comparison to a smooth tube, it was observed by Shchelkin that a consistent reduction in run-up distance to DDT occurred when obstacles were used. It was found, as well, that hydrocarbon-air mixtures (which were previously believed to be undetonable) can transition to detonation in an obstacle field; however, the steady state velocities were found to be lower than the theoretical Chapman-Jouguet (C-J) detonation velocity. Guénoche $^{4}$ later repeated Shchelkin's experiments to study the influence of different obstacle configurations (i.e. different spiral pitch and wire thickness) on the propagation of combustion waves. Velocity deficits of about $40 \%$ from the theoretical C-J value were also reported. These detonation-like waves in obstacle-filled tubes that propagate at velocities much lower than the C-J value were coined as "quasi-detonations" by Zel'dovich. ${ }^{5}$ 


\subsection{Motivation}

In the last twenty-five years after a three decade hiatus, there has been renewed interest (both experimentally and numerically) in turbulent flame acceleration by obstacles. Quasi-detonations and even high speed turbulent deflagrations pose serious threats in industrial settings where accidental fuel-air explosions can occur. The ability to accurately and realistically estimate the significant over-pressures generated by obstacle accelerated flames is crucial in the effort to assess industrial hazards. Numerous computer codes (e.g. REAGAS, FLACS, EXSIM, and COBRA) have been developed to achieve this end. ${ }^{6}$ In these codes, the resolution of the computational grid is rather coarse and, thus, sub-models are required to describe the effect of obstacles, the turbulent flow, and the combustion processes that occur on much smaller length scales. These sub-models, however, do not accurately describe supersonic combustion controlled by auto-ignition and by shock wave interactions. ${ }^{7}$ The turbulence sub-models are valid only for incompressible flows, while the combustion sub-models are based on a laminar flamelet. Nonetheless, accurate estimates can be made for high speed turbulent deflagrations and quasi-detonations - but only when appropriate empirical constants are chosen arbitrarily to fit experimental data. It is for this reason, therefore, that the propagation mechanism of high speed turbulent deflagrations must be clarified through experimental work.

\subsection{Previous work}

Previous experiments in circular tubes with periodically spaced orifice plate obstacles have established that there exist distinct steady state propagation regimes (e.g. quasi-detonation, high speed turbulent deflagration, "choking," and slow turbulent deflagration). ${ }^{8}$ These regimes are characterized by the maximum steady state velocities attained by the propagating flame after an initial acceleration phase. The maximum steady state velocity is of particular interest in understanding the propagation mechanisms since this is where there exists a critical balance between the positive flame acceleration mechanisms and the negative quenching mechanisms of turbulent combustion waves. ${ }^{9}$ It was found that quasi-detonation velocities were observed in 
fuel-air mixtures when $d / \lambda \geq 1$ (where $d$ is the orifice diameter and $\lambda$ is the characteristic detonation cell size). At a critical concentration where $\mathrm{d} / \lambda \approx O[1]$, there is sharp transition to the "choking" regime where the steady state velocity drops to the magnitude of the sound speed of the combustion products. Thus, in very small diameter tubes or in insensitive mixtures like methane-air, it was found that the quasi-detonation regime was not observed.

The existence of the different steady state propagation regimes establishes that there is a distinct propagation mechanism associated with each regime, and the abrupt transitions that are observed at critical concentrations are indicative of sudden changes in the propagation mechanisms.

\subsection{Limitation of previous work}

The propagation mechanism of high speed turbulent deflagrations in rough-walled or obstacle-filled tubes is still unclear. In the previous study in circular tubes with orifice plate obstacles, the terminal steady state flame velocities that were used to characterize the propagation regimes were averaged values taken over relatively long distances of propagation of a few tube diameters (or obstacle spacings). ${ }^{8}$ As a consequence, valuable information on the propagation mechanism was lost since the local velocity fluctuations that contain information about the phenomenon were not considered. A more detailed investigation into local velocity variations is needed (especially at the transition limit between adjacent regimes) in order to achieve a better understanding of the propagation mechanisms.

However, local velocity fluctuations can not be adequately studied using an obstacle configuration of regularly spaced orifice plates since it does not have sufficient generality for producing small scale turbulence by flow randomization. A tube filled with periodically spaced orifice plates is, essentially, a series of interconnected explosion chambers. As the mixture in one chamber reacts rapidly and explodes, the abrupt pressure rise can choke the flow at the orifice. The subsequent venting of the combustion products at the local sound speed produces a sonic jet into the downstream chamber. The combustion front gets convected along with the jet and propagates accordingly at the local sound speed of the combustion products. This mechanism is the basis of defining 
the so-called "choking" regime. ${ }^{8}$ It is clear that the propagation velocities in this regime do not accurately represent actual turbulent burning rates of high speed turbulent deflagrations. Therefore, any fluctuation from obstacle to obstacle does not accurately represent the actual fluctuations associated with an intense turbulent combustion zone.

\subsection{Classification of steady state combustion waves}

In general, it is difficult to precisely define the different regimes on the basis of the propagation velocities alone since an abrupt transition between the different regimes may not always exist. This is the case with certain obstacle configurations where it is observed that the high speed turbulent deflagrations and the quasi-detonations appear in a continuous range of velocities without an abrupt transition. For example, in porous media or in a packed bed of spherical particles, ${ }^{10,11}$ it is observed that there is a spectrum of steady state velocities ranging from very near the theoretical Chapman-Jouguet (C-J) detonation velocity to as low as $30 \%$ of the C-J value without any distinguishable transition. On the other hand, in other obstacle configurations such as periodically spaced orifice plates, a transition between the two regimes is well defined. The absence of distinct regimes when using certain obstacle configurations indicates that the propagation mechanisms are dependent on boundary conditions and implies that certain regimes are particular to the obstacle geometry used. Furthermore, without a clear distinction in the propagation velocities, the propagation mechanisms of quasi-detonations and of high speed turbulent deflagrations can not be dichotomized. Nevertheless, a general differentiation between the quasi-detonation regime and the high speed turbulent deflagration regime can be made if one considers the auto-ignition limit to separate detonative and supersonic deflagrative combustion. In other words, the averaged propagation velocity of a quasi-detonation should be such that the post-shock temperature of a C-J detonation at that velocity is above the auto-ignition limit of the mixture.

In the present investigation, the quasi-detonation regime will be denoted as one in which the velocity is in the range of the C-J detonation velocity to about half the C-J value. In general, shock temperatures in the quasi-detonation velocity range are above the auto-ignition limit. This is then followed by the high speed turbulent deflagration regime where the flame velocities vary between the sound speed of the unburned mixture 
$(\approx 350 \mathrm{~m} / \mathrm{s})$ to about the sound speed of the combustion products (which corresponds to about half the theoretical C-J value). According to this definition, high speed turbulent deflagrations are supersonic waves relative to the unburned mixture. Finally, the slow turbulent deflagration regime is the subsonic regime with propagation velocities less than the speed of sound of the unburned mixture.

\subsection{Present work}

In the present study, propagation velocities and their fluctuations (particularly at the transition between the different regimes) are investigated in order to provide insight into the propagation mechanism of high speed turbulent deflagrations. Closely spaced staggered vertical cylindrical rod obstacles are used to create a more "uniform" turbulent flow field by reducing the turbulent length scales (compared to the reaction zone) in both the transverse and the longitudinal directions. In this manner, more meaningful local propagation velocities and their fluctuations can be obtained over a distance of the order of a single tube diameter. 


\section{Chapter 2 Experimental details}

\subsection{Experimental apparatus}

In the present experiment, a $7 \mathrm{~m}$ long $30 \mathrm{~cm} \times 30 \mathrm{~cm}$ square cross-sectioned steel tube was used. The obstacle configuration consisted of a staggered arrangement of vertical cylindrical rods in alternating rows of 3 rods and of 2 rods (see Fig. 1). Each row was spaced half a tube width (or "diameter") apart. Two sets of obstacles were used. For the larger obstacle array, the diameter of the cylindrical rods was $d=3.4 \mathrm{~cm}$, giving an average blockage ratio (BR) of 0.41 . For the smaller obstacle array, the cylindrical rods were $d=2.54 \mathrm{~cm}$ in diameter for an average BR of 0.19 . The blockage ratio is defined as the ratio of the blocked cross-sectional area to the total cross-sectional area. Since the number of cylindrical rods alternate between 3 and 2, giving different blockage ratios, an averaged value was used. A photograph of the obstacle array next to the combustion tube is shown in Fig. 2.

A driver section was used to provide a strong initiation source so that the final steady flame speed could be attained in a short distance. This was required for less sensitive mixtures where a longer distance of flame acceleration was required to reach the final steady state. The driver section consisted of a $1.1 \mathrm{~m}$ long steel tube with an inner diameter of $14.5 \mathrm{~cm}$. Orifice plates with $\mathrm{BR}=0.44$ that span the entire length of the driver section were spaced one tube diameter apart to promote rapid flame acceleration. A 0.003 " thick Mylar diaphragm separated the driver from the main tube, which allowed a more sensitive mixture to be used in the driver section to obtain a strong initiation. A schematic of the experimental set-up is shown in Fig. 3. Ignition of the mixture in the driver section was achieved by means of an electric match. It was found that without the driver, the terminal velocities for the less sensitive mixtures could only be achieved in the last few meters of the tube. However, with the driver section, the final steady state velocity could be reached within a couple of meters (even for the most insensitive mixtures studied), thus allowing ample distance of flame travel in the steady state regime to be observed. 


\subsection{Mixture selection and preparation}

Four different fuels (hydrogen, ethylene, propane, and methane), which encompass a wide range of detonation sensitivities, were used in the present study. Air was used as the oxidizer. Of the four fuels tested, hydrogen is the most sensitive. The kinetics of its reaction is well-established. Propane is representative of the majority of the higher hydrocarbon fuels whereas methane is unique amongst the alkane group as it has similar energetics but very slow induction kinetics. In some experiments, ethane was also used in an attempt to sensitize methane-air mixtures.

For any given experiment, the tube was initially evacuated and then filled by the method of partial pressures to obtain the desired concentrations of fuel and of air. The pressure was monitored by a Heise gauge with a rated accuracy of $\pm 0.1 \%$ F.S. or \pm 1 torr. The initial pressure of all the experiments in the present study was 1 atmosphere (760 torr). Once the tube was filled, the mixture was re-circulated through the system using a bellows-type re-circulation pump for a sufficient amount of time in order to ensure a homogenous mixture. In the driver section, a stoichiometric mixture of hydrogen-air was used and was prepared in the same manner as the mixture in the main tube. A separate bellows re-circulation pump was used to ensure a homogenous composition within the driver.

\subsection{Diagnostics}

Arrival times of the combustion front were monitored by ionization probes spaced one tube diameter apart along the length of the tube. A typical oscilloscope trace of signals from the ionization probes is shown in Fig. 4. Each drop in voltage corresponds to the arrival time of the combustion front. Since the ionization probes are spaced equidistantly, the flame acceleration process can be seen in Fig. 4 as indicated by the decreasing time interval between voltage drops. The time intervals become the same when the steady state velocity is attained by the combustion wave. The time of arrival permits the flame trajectory to be determined, and the slopes of the flame trajectory can be used to obtain a global average of the steady state velocity. Local flame velocities can also be calculated between arrival times of adjacent ionization probes. 
Both long and short ionization probes were used to measure arrival times. It was found previously with orifice plate obstacles that short ionization probes located near the wall of the tube would record spurious arrival times due to a fluctuating flame shape (as the flame would jet between the tube wall and the orifice plates). Long ionization probes that extend to the center of the tube were required to obtain meaningful arrival times. However, in the present study, it was found that long and short ionization probes gave similar results. This suggests that the obstacle array of staggered vertical rods creates a more "uniform" turbulent reaction zone without the "jetting" effect due to the circular orifice plate obstacles used in previous studies. ${ }^{8}$

The pressure rise associated with the combustion wave was monitored by a PCB pressure transducer. In general, the pressure records for supersonic combustion waves show sharp "spikes" about a mean value. The pressure fluctuations are due to the ensemble of transverse shock waves generated in the combustion zone by the obstacles. The mean pressure rise corresponds to the constant volume explosion pressure of the mixture. A typical pressure trace for a high speed turbulent deflagration is shown in Fig. 5 for a stoichiometric mixture of methane-air. The average pressure rise is found to be about 7.5 bar, which corresponds closely to the constant volume explosion pressure $\left(\mathrm{P}_{\mathrm{CV}} \approx 8.9\right.$ bar $)$ for stoichiometric methane-air mixtures. The pressure profile for quasi-detonations are found to be very similar to those for high speed turbulent deflagrations. In Fig. 6, a typical pressure trace of a quasi-detonation for stoichiometric propane-air is shown. The average pressure rise is found to be about $8.1 \mathrm{bar}$, which also closely corresponds to the constant volume explosion pressure for stoichiometric propane-air $\left(\mathrm{P}_{\mathrm{CV}} \approx 9.5\right.$ bar). The constant volume explosion pressure is about 8 to 10 bar for stoichiometric mixtures of the common hydrocarbon fuels with air. The pressure record for a slow turbulent deflagration is shown in Fig. 7. In contrast to the pressure profiles for high speed turbulent deflagrations and for quasi-detonations, the rise time is long and gradual. The pressure rise corresponds to only a fraction of an atmosphere. 


\section{Chapter 3 Results and discussion}

The different propagation regimes (e.g. quasi-detonation, high speed turbulent deflagration, and slow turbulent deflagration) that were observed in previous investigations (in different tube geometries and obstacle configurations) are also found in the present investigation. However, the magnitude of the steady state flame velocities are found to differ from those observed previously. The sharp transitions from one regime to another are also found to occur at different mixture compositions.

\subsection{General observations}

A typical flame trajectory for the case of a stoichiometric propane-air mixture is shown in Fig. 8. A weak electrical match was used for ignition instead of the driver section for this particular set of experiments. It can be seen that after a rapid initial acceleration of the flame for about $2 \mathrm{~m}$, the combustion wave reached a steady state velocity and propagated henceforth at this velocity. An overall averaged value of the steady state velocity can be obtained from the mean slope of the $\mathrm{x}$-t trajectory. For the three separate experiments shown in Fig. 8, it can be seen that the time for the initial acceleration of the combustion wave (until a steady state is reached) differs only slightly. This is indicative of the reproducibility of the transient flame acceleration process when obstacles are used (in a smooth tube, in general, the run-up distance to steady state is highly irreproducible). Once a steady state is reached, the slopes of the three trajectories of the separate experiments are also found to be almost identical, giving velocities of $1596 \mathrm{~m} / \mathrm{s}, 1610 \mathrm{~m} / \mathrm{s}$, and $1613 \mathrm{~m} / \mathrm{s}$. In general, the averaged steady state velocities are found to be highly reproducible from experiment to experiment, especially for the more sensitive near-stoichiometric mixtures. It should be noted that velocity fluctuations are not manifested in the averaged velocities when obtained in this manner since a straight line can be made to fit relatively well with the experimental data once a steady state is reached.

Apart from a single value of the global averaged velocity obtained from the flame trajectory, local flame velocities along the tube can also be obtained by dividing the 
distance between adjacent ionization probes by the difference in arrival times. The variation of the local velocity along the tube for the same case of the stoichiometric propane-air mixture is shown in Fig. 9. Here, the flame can be seen to accelerate to steady state after propagating for a slightly longer distance of about $2.5 \mathrm{~m}$. After the steady state is reached, the local velocity also appears to exhibit some fluctuations about an averaged value. The averaged value obtained from the local velocity (about $1630 \mathrm{~m} / \mathrm{s}$ ) is found to correspond closely to the overall averaged value determined from the flame trajectory over the entire length of steady flame propagation. It is interesting to note that there does not appear to be an overshoot in the velocity, which is characteristic of DDT in smooth tubes at the onset of detonation. This suggests that in a rough-walled or obstacle filled tube, the onset of detonation is a gradual process without an abrupt change in the propagation mechanism from deflagration to detonation.

When a driver section was used for ignition instead of an electric match, the steady state was reached within a much shorter distance of flame travel. This is shown in Fig. 10 for a rich mixture of propane-air. The powerful driver initiates an overdriven combustion wave, but the velocity decays from over $2000 \mathrm{~m} / \mathrm{s}$ to the steady state velocity of around $1600 \mathrm{~m} / \mathrm{s}$ after $2.5 \mathrm{~m}$ of travel. Without the use of the driver section, the steady state can only be reached after a distance of $5 \mathrm{~m}$ for this mixture. However, the same value for the final propagation velocity is found indicating that the steady state is independent of the initial flame acceleration phase.

\subsection{Steady state regimes}

\subsubsection{Quasi-detonation regime}

For a sensitive stoichiometric mixture of hydrogen-air, quasi-detonations are found to propagate at velocities very close to the theoretical C-J detonation velocity of about $2000 \mathrm{~m} / \mathrm{s}$ in the present obstacle configuration. The variation of velocity with distance for stoichiometric hydrogen-air for $\mathrm{BR}=0.41$ is shown in Fig. 11a. Although there appears to be some small fluctuations in the local velocity $(\approx 250 \mathrm{~m} / \mathrm{s}$, peak to

peak), it can be seen from the corresponding velocity histogram (Fig. 11b) that the velocity distribution peaks sharply near the C-J value with little deviation from the peak 
value. The velocity fluctuations that are associated with the quasi-detonation regime are found to be similar to those observed in the "rapid fluctuation" mode for near-limit detonations in smooth tubes. ${ }^{12}$ In this unstable mode in smooth tubes, the detonation propagates at sub-C-J values, exhibiting irregular oscillations in amplitude and in frequency. The variation of the local velocity with distance that is presently observed for stoichiometric hydrogen-air is typical of quasi-detonations and is also observed for hydrogen-air mixtures near the lean and the rich limits of the quasi-detonation regime (Figs. 12 and 13). Similar results are also found for ethylene-air and for propane-air in the quasi-detonation regime (Figs. 14 to 19).

The variation of the final steady state velocity as a function of mixture composition for hydrogen-air, ethylene-air, and propane-air mixtures are shown in Figs. 20, 21 and 22, respectively. For each of these fuel-air mixtures, the results for both $\mathrm{BR}=0.41$ and $\mathrm{BR}=0.19$ are shown together with the previous results obtained in a circular tube with orifice plate obstacles of a similar blockage ratio $(B R=0.43)$. The theoretical C-J detonation velocity as well as the sound speed of the combustion products are also shown for comparison in each case. For hydrogen-air with the present obstacle configuration $(\mathrm{BR}=0.41)$, the quasi-detonation regime is observed in the range of $0.52<\phi<3.88$ ( $18 \%$ to $62 \%$ hydrogen). For ethylene-air and for propane-air, the quasi-detonation regime is observed for a range of $0.6<\phi<2.2$ and of $0.7<\phi<1.45$, respectively (where $\phi$ is the equivalence ratio). For the three fuel-air mixtures, the velocities of the quasi-detonation regime are found to be only slightly lower than the theoretical C-J value and demonstrate only a small dependence on the equivalence ratio. This is similar to the dependence of the theoretical C-J detonation velocity on equivalence ratio, indicating that quasi-detonations are also governed by the energetics of the mixture. In comparison to the previous results for quasi-detonations obtained in a circular tube, the quasi-detonation velocities are found to be higher with the present obstacle configuration. This indicates that the more evenly distributed smaller scale obstacles produce less perturbations in the reaction zone of a quasi-detonation. In Figs. 20 to 22 , it can also be seen that when the blockage ratio is reduced to 0.19 , there does not appear to be any significant changes in quasi-detonation velocities for any of the fuels tested. This suggests that blockage due to the evenly distributed obstacles does not 
have a pronounced effect on the propagation mechanism, and it is the energetics of the mixture that controls the propagation velocity of quasi-detonations.

The deficit in the theoretical C-J velocity that is observed for quasi-detonations is probably due to the randomization of the mean flow and to the diffraction of the detonation around the obstacles. The detonation is repeatedly attenuated by diffraction but is also repeatedly re-initiated by auto-ignition due to strong shock reflections from the tube wall and from the obstacles. This mechanism is well-illustrated in the Schlieren photography of quasi-detonations of Teodorcyk et al. ${ }^{13}$

\subsubsection{High speed turbulent deflagration regime}

The steady high speed turbulent deflagration regime is observed for only hydrogen-air mixtures in the composition range of $3.88<\phi<5.55$ (62\% to $70 \%$ hydrogen) as shown in Fig. 20. Although there may appear to be a significant drop in the steady state velocity at a mixture composition of $\phi \approx 3.88$ ( $62 \%$ hydrogen), the transition from the quasi-detonation regime to the high speed turbulent deflagration regime is quite gradual (as the velocity decreases steadily from $2000 \mathrm{~m} / \mathrm{s}$ to $1000 \mathrm{~m} / \mathrm{s}$ ). This implies that a sudden change in the propagation mechanism does not occur. In Fig. 23a, a high speed turbulent deflagration is shown to propagate steadily at an averaged value around $1000 \mathrm{~m} / \mathrm{s}$. There exists very little fluctuations in the local flame velocity as was also observed in the quasi-detonation regime. The corresponding velocity histogram in Fig. 23b shows very little deviation from the average, which is $50 \%$ of the theoretical C-J detonation velocity. The steady high speed turbulent deflagration regime is observed only for rich hydrogen-air mixtures for both $\mathrm{BR}=0.41$ and $\mathrm{BR}=0.19$.

In general, a propagation mechanism can be considered to be comprised of an ignition mechanism and of a driving mechanism. The ignition mechanism represents the means by which the unburned mixture in the flame zone is ignited (e.g. heat and mass transport from the flame zone or auto-ignition via adiabatic compression from the leading shock). The driving mechanism for the combustion wave accounts for how the flame zone is advanced into the unburned mixture. The propagation of the high speed turbulent deflagrations at such high supersonic velocities indicates that the turbulent diffusional heat and mass transport mechanism of a regular turbulent flame is perhaps too slow to be 
responsible for the fast burning rates that are required. It has also been shown in the Schlieren photography of Teodorcyk et al. ${ }^{14}$ that the leading shock plays a minor role in the propagation of high speed turbulent deflagrations. This suggests that it is rapid turbulent mixing (promoted by the obstacles) of the hot combustion products with the unburned mixture that leads to adiabatic auto-ignition, in contrast to the classical ZND detonation where auto-ignition is achieved via adiabatic compression by the leading shock. It has been demonstrated by Atkinson et al. ${ }^{15}$ that, for hydrogen-air, there exists a critical auto-ignition temperature of $1100 \mathrm{~K}$ below which an induction time can no longer be defined. In Fig. 24, the post-shock temperature of a C-J detonation is plotted as a function of equivalence ratio. From this figure, it can be seen that this auto-ignition limit corresponds to $\phi \approx 5$ (68\% hydrogen). ${ }^{16}$ Therefore, for high speed turbulent deflagrations in the range of $5<\phi<5.55$ (68\% to $70 \%$ hydrogen), auto-ignition due to shock heating is not possible and, hence, ignition in high speed turbulent deflagrations is perhaps controlled solely by turbulent mixing.

Near the auto-ignition limit, there are essentially two competing reactions: ${ }^{15,17}$

$$
\begin{aligned}
& \mathrm{H}+\mathrm{O}_{2} \rightarrow \mathrm{HO}+\mathrm{O} \\
& \mathrm{H}+\mathrm{O}_{2}+\mathrm{M} \rightarrow \mathrm{HO}_{2}+\mathrm{M}
\end{aligned}
$$

It was also found by Atkinson et al. ${ }^{15}$ that above a shock temperature of $1350 \mathrm{~K}$ at $\phi=3$ (55\% hydrogen), the rate of the chain branching reaction [1] is much greater than the rate of the recombination reaction [2]. This permits the rapid consumption of hydrogen and of oxygen as well as the rapid production of large concentrations of free radicals, which is characteristic of detonations. As the shock temperature decreases to $1100 \mathrm{~K}$ (corresponding to the range of $3<\phi<5$ or $55 \%$ to $68 \%$ hydrogen), the rate of the recombination reaction [2] increases, thereby creating an increasing inhibiting effect as the hydroperoxy $\left(\mathrm{HO}_{2}\right)$ radical destroys $\mathrm{H}$ radicals. Not only are free radicals being produced in smaller concentrations, fuel and oxidizer are also being consumed at a diminishing rate. It is perhaps for this reason that no sharp transition is observed from the quasi-detonation regime to the high speed turbulent deflagration regime. As the shock temperature approaches the auto-ignition limit, shock-induced auto-ignition is increasingly inhibited by the recombination reaction [2], and there is a gradual transition as auto-ignition becomes more heavily dependent on turbulent mixing. 
Since the mean pressure rise in high speed turbulent deflagrations corresponds to the constant volume explosion pressure, there is also a certain degree of temperature rise associated with the adiabatic compression across the flame. Furthermore, a local temperature rise can result from compression across the transverse shock waves that reflect from the obstacles and the tube wall. Strong turbulent mixing enhancement can also be effected by the strong pressure fluctuations of the transverse shock waves, providing an efficient means of vorticity production and turbulent energy dissipation in the reaction zone. The resulting turbulent mixing rate can be much greater than that due to the conventional turbulent mixing mechanism of low speed turbulent flames. The driving mechanism for supersonic high speed turbulent deflagrations also differs from that of ordinary low speed turbulent flames controlled by diffusion and transport. The expansion of the combustion products can provide the driving mechanism for high speed turbulent deflagrations similar to that for classical detonations.

Thus, the propagation mechanism of high speed turbulent deflagrations is essentially similar to that of a detonation wave. It is, therefore, difficult to draw a sharp distinction between C-J detonations, quasi-detonations, and high speed turbulent deflagrations if they all essentially propagate via the same mechanisms and differ only in the degree which auto-ignition is achieved via turbulent mixing and adiabatic shock compression.

\subsubsection{Transition to the slow turbulent deflagration regime}

An abrupt transition from the quasi-detonation regime (or the high speed turbulent deflagration regime in rich hydrogen-air mixtures) to the slow turbulent deflagration regime is observed for the three fuel-air mixtures that are investigated. For rich hydrogen-air mixtures, the transition from the high speed turbulent deflagration regime occurs at a concentration of about $\phi=5.55$ ( $70 \%$ hydrogen) for both $\mathrm{BR}=0.41$ and $\mathrm{BR}=0.19$ (Fig. 20). For ethylene-air (Fig. 21) and propane-air (Fig. 22) mixtures with both $\mathrm{BR}=0.41$ and $\mathrm{BR}=0.19$, the transition from the quasi-detonation regime to the slow turbulent deflagration regime occurs directly for rich mixtures at $\phi=2.2$ and at $\phi=1.45$, respectively. The flame speeds in the slow turbulent deflagration regime are found to be of the order of $200 \mathrm{~m} / \mathrm{s}$. These low speed deflagrations are observed for both 
$\mathrm{BR}=0.41$ and $\mathrm{BR}=0.19$. In Table 1 , correlations of the different physical length scales of the apparatus with $\lambda$ are shown for the three fuels at the lean and at the rich transition limits between the high speed turbulent deflagration and the slow turbulent deflagration regimes. It can be seen that correlations of the transverse spacing between adjacent cylindrical rods, $w$, and of the diameter of the cylindrical rods, $\mathrm{d}$, with $\lambda$ yield values much smaller than 1. Instead, the critical compositions at the transition limits all correspond to $\mathrm{D} / \lambda \approx O[1]$ where the detonation cell size is of the order of the tube diameter, D. Hence, with the present obstacle configuration, it is the largest physical length scale of the experiment of the tube diameter (and not the length scale of the obstacle spacing or of the obstacle dimension) that governs the transition from the quasidetonation or high speed turbulent deflagration regime. The $\mathrm{D} / \lambda \approx O[1]$ limit criterion also corresponds to the deflagration to detonation transition (DDT) criterion in smooth tubes proposed earlier by Lee. ${ }^{18}$ This suggests further that C-J detonations, quasidetonations and high speed turbulent deflagrations are, in fact, the same phenomenon and differ only in the degree which shock heating or turbulent mixing controls auto-ignition in the reaction zone.

\begin{tabular}{|c|l|c|c|c|}
\cline { 3 - 5 } \multicolumn{2}{c|}{} & $\frac{w}{\lambda}$ & $\frac{d}{\lambda}$ & $\frac{D}{\lambda}$ \\
\hline \multirow{2}{*}{ Hydrogen-air } & Lean limit & $\sim 0.24$ & $\sim 0.10$ & $\sim 1.12$ \\
\cline { 2 - 5 } & Rich limit & 0.20 & 0.08 & 0.917 \\
\hline \multirow{2}{*}{ Ethylene-air } & Lean limit & 0.29 & 0.12 & 1.34 \\
\cline { 2 - 5 } & Rich limit & 0.27 & 0.11 & 1.22 \\
\hline \multirow{2}{*}{ Propane-air } & Lean limit & 0.28 & 0.12 & 1.3 \\
\cline { 2 - 5 } & Rich limit & 0.52 & 0.22 & 2.37 \\
\hline
\end{tabular}

Table 1: Correlation of different physical length scales of the experimental apparatus with $\lambda$ at transition limits. 


\subsubsection{Absence of the "choking" regime}

In contrast to the results obtained previously in a circular tube with orifice plate obstacles, the so-called "choking" regime appears to be absent with the present obstacle configuration. In Fig. 20, it can be seen that for the case of rich hydrogen-air mixtures only, a high speed turbulent deflagration regime with velocities close to the sound speed of the combustion products is observed in lieu of a choking regime in a narrow composition range of $4.23<\phi<5.55$ (64\% to $70 \%$ hydrogen). For ethylene-air (Fig. 21) and propane-air mixtures (Fig. 22), the transition to the slow turbulent deflagration regime occurs directly from the quasi-detonation, thereby bypassing the choking regime altogether. This suggests the "choking" regime may be unique to only orifice plate geometries where the flame essentially propagates as a result of the sequential venting of the combustion products at the local sonic speed through choked orifices.

This phenomenon is well-illustrated in a sequence of computer generated images of flame acceleration through a series of regularly spaced orifice plates using the Vortex Dynamics Code (Fig. 25a). ${ }^{19}$ As the flame advances, shear layers (indicated by the areas of increased vorticity) are generated at the orifice plates by the displacement flow ahead of the propagating flame. By the fourth frame, it can be seen that the flame gets convected into the gradient field and that the tip of the flame is stretched as it is vented through the orifices. In the last frame, it is evident that the flame is jetted through successive orifices without complete burnout in each chamber. This is corroborated by the Schlieren photography of Kuznetsov et al. (Fig. $25 \mathrm{~b}$ ). ${ }^{20}$ For a $70 \%$ hydrogen mixture with air $(\phi=5.55)$, it is clear that the propagation of the flame in the "choking" regime is due to a convective jetting effect rather than to an actual turbulent combustion mechanism.

\subsection{Unstable regimes at the transition limit}

Highly unsteady flame propagation is observed near critical compositions corresponding to the transition from the quasi-detonation regime to the slow turbulent deflagration regime. Unstable behaviour is observed only at the lean transition limit. The mixture composition for the three fuels at the transition to the slow turbulent deflagration 
regime is found to be governed by the criterion $\mathrm{D} / \lambda \approx 1$ for each case (see Table 1 ). In the unsteady regime, the velocity fluctuations can be quite significant. In Figs. 20 to 22, the magnitude of the fluctuations is denoted by a dashed vertical line for the three different fuels. For lean hydrogen-air mixtures in the range of $0.32<\phi<0.52(12 \%$ to $18 \%$ hydrogen), the magnitude of the velocity fluctuations is observed to be between $1000 \mathrm{~m} / \mathrm{s}$ and $2500 \mathrm{~m} / \mathrm{s}$. The local velocity variation along the length of the tube for mixtures in this unstable range of near-limit mixture composition is shown in Fig. 26a for hydrogen-air. The velocity fluctuations do not appear to have periods of oscillation that can be correlated to either a physical or a chemical length scale of the experiment. This indicates that the fluctuations are not controlled by regularly spaced obstacles but are due to the non-steady combustion process in the reaction zone itself. The corresponding velocity histogram for the unstable deflagration near the transition limit is shown in Fig. 26b. It can be seen that there is a wide velocity distribution, albeit that the average peak value is still found to be only slightly lower than the C-J detonation velocity (as in galloping detonations). This is also typical of ethylene-air mixtures at $\phi=0.6$ and of propane-air mixtures at $\phi=0.7$ where significant velocity fluctuations are also observed near the transition limit from the quasi-detonation regime to the slow turbulent deflagration regime. The velocity variation with distance and the corresponding velocity histograms are shown in Figs. 27 to 28.

The large velocity fluctuations observed near the lean transition limit from the quasi-detonation and the high speed turbulent deflagration regime to the slow turbulent deflagration regime can be credited to the quenching and re-ignition of large pockets of

\begin{tabular}{|c|c|c|}
\hline Fuel-air mixture & $\begin{array}{c}\text { Explosion } \\
\text { quenching } \\
\text { Diameter }(\mathrm{mm})\end{array}$ & MESG (mm) \\
\hline Hydrogen & 1.2 & 0.28 \\
\hline Ethylene & 18 & 0.7 \\
\hline Methane & 40 & 0.97 \\
\hline Propane & 60 & 1.15 \\
\hline
\end{tabular}

Table 2: Explosion quenching diameter and MESG for different stoichiometric fuel-air mixtures. 
unburned reactants. It has been established that rapid mixing can result in quenching of a turbulent flame. In a previous study by Thibault et al., ${ }^{21}$ it was found that the quenching diameter for the transmission of the explosion products through an orifice plate downstream into unburned mixture is more than an order of magnitude greater than the normal flame quenching distance or the MESG (Maximum Experimental Safety Gap). In Table 2, the quenching diameters are shown for different stoichiometric fuel-air mixtures compared to the MESG. In these experiments by Thibault et al., the quenching diameter was found to correlate with the pressure upstream of the orifice, which corresponds to values of the order of the constant volume explosion pressure (about 6 atm for propane-air and methane-air mixtures and around $9 \mathrm{~atm}$ for ethylene-air mixtures). Therefore, the venting of the products downstream is via a choked sonic jet. It was also proposed in this study that quenching occurs when the characteristic reaction time is long compared to the characteristic mixing time. The quenching limit was correlated to:

$$
\frac{\tau_{\text {reaction }}}{\tau_{\text {mixing }}} \approx O[1]
$$

In the case of relatively insensitive mixtures, the turbulent high speed deflagration is more sensitive to local quenching as the induction time increases. However, the energetics of less sensitive mixtures are still sufficiently high to produce a high pressure rise (of the order of the constant volume explosion pressure) to drive the combustion front at supersonic speeds via the expansion of the high pressure combustion products. When the reaction rate of these near-limit mixtures becomes sufficiently slow, partial quenching may occur in the intense turbulent mixing zone. When this occurs, there is a decrease in the pressure rise of the combustion products and, hence, a corresponding decrease in the flame velocity. However, as the quenched volume of unburned mixture swept downstream re-ignites at a later time, the energy that is released sends a compression pulse which catches up to the leading combustion front. This causes it to accelerate and gives rise to the observed pulsations. It appears that the unstable transition occurs at a distinct concentration for ethylene-air and propane-air in contrast to hydrogen-air where there is a larger range of fuel composition exhibiting significant velocity fluctuations. Although it is not clear why this occurs, it does suggests that high 
speed turbulent deflagrations for lean hydrogen-air mixtures can be quenched more readily than those for lean ethylene-air and propane-air mixtures.

These oscillations can be visualized in the streak photography of Lieberman and Lee, ${ }^{22}$ taken for low pressure acetylene-oxygen mixtures in a circular tube with orifice plate obstacles. A streak photograph of a quasi-detonation with very little fluctuations in velocity is shown in Fig. 29a. In contrast, a streak photograph taken at the transition from the quasi-detonation regime to the "choking" regime exhibits significant fluctuations (Fig. 29b). The combustion wave is observed to accelerate and to decelerate continually. The luminosity is also seen to vary along trajectory. These fluctuations in velocity may arise as a result of the quenching of the combustion front and the subsequent explosion of quenched volumes of unburned reactants downstream. For the case with high argon dilution (75\% argon), the large fluctuations are not observed to occur (Fig. 29c) since highly diluted argon mixtures are perhaps not as susceptible to local quenching due to turbulent mixing. In these mixtures, the combustion wave is less sensitive to temperature perturbations in the reaction zone and are, therefore more stable.

\subsubsection{Absence of unstable regimes at rich transition limits}

Near the transition limit on the rich side, fluctuations are not observed at all for the three fuels investigated, which indicates that the combustion front is not as sensitive to local quenching for rich mixtures. This may be due to the higher concentration of free radicals and non-equilibrium active species in the reacting gases and in the combustion products of rich mixtures. In the previous study of Carnasciali et al. ${ }^{23}$ on detonation initiation by a turbulent jet of combustion products, it was found that a higher concentration of free radicals in a turbulent jet of combustion products can facilitate the direct initiation of a detonation via mixing. From a thermodynamic equilibrium code (STANJAN), the concentration of $\mathrm{H}$ radicals at the rich limit is about an order of magnitude larger than the $\mathrm{H}$ radical concentration at the lean limit (Table 3). A higher concentration of $\mathrm{H}$ radicals in rich mixtures facilitates the very important chain branching reaction. As well, it was also reported by Atkinson et al. ${ }^{15}$ that calculated induction times for rich mixtures are shorter than those for lean mixtures, which was credited to enhanced $\mathrm{HO}_{2}$ and $\mathrm{H}_{2} \mathrm{O}_{2}$ formation on the lean side. Therefore, local quenching of the combustion 


\begin{tabular}{|c|c|c|}
\cline { 2 - 3 } \multicolumn{1}{c|}{} & \multicolumn{2}{c|}{$\begin{array}{c}\text { Concentration of H radicals } \\
\text { (mole fraction) }\end{array}$} \\
\cline { 2 - 3 } & $\frac{D}{\lambda} \approx 1$ (lean limit) & $\frac{D}{\lambda} \approx 1$ (rich limit) \\
\hline Hydrogen-air & $1.747 \times 10^{-4}$ & $1.006 \times 10^{-3}$ \\
\hline Ethylene-air & $1.846 \times 10^{-4}$ & $2.303 \times 10^{-3}$ \\
\hline Propane-air & $2.784 \times 10^{-4}$ & $3.264 \times 10^{-3}$ \\
\hline
\end{tabular}

Table 3: Concentration of $\mathrm{H}$ radicals at transition limits for different fuel-air mixtures.

front is less likely to occur for rich mixtures and, hence, the large velocity fluctuations due to quenching and re-ignition of pockets of unburned gases are not observed.

\subsubsection{Effect of blockage ratio on the unstable regime}

A reduction in the blockage ratio appears to influence only the unsteady propagation of lean mixtures of ethylene-air and of propane-air near the transition limit of $\mathrm{D} / \lambda \approx 1$. A comparison of the velocity variation with distance is shown in Figs. 30 and 31 for ethylene-air and propane-air, respectively. For a $B R=0.19$, quasi-detonation velocities around $1500 \mathrm{~m} / \mathrm{s}$ are observed in contrast to the averaged velocities of about $1000 \mathrm{~m} / \mathrm{s}$ with large fluctuations that are observed for the same mixtures with a $\mathrm{BR}=0.41$. This is not surprising since there are less momentum losses to the smaller obstacle array. The smaller obstacle array $(B R=0.19)$ generates finer scale turbulence, which means there is a shorter characteristic reaction time. As a result, the combustion front may not be as easily quenched, and the pressure rise due to the combustion products (and the high flame velocities) can be maintained. In contrast to the case with the large obstacle array $(\mathrm{BR}=0.41)$, smaller pockets of unburned mixture are trapped behind in the reaction zone and have a shorter residence time before they explode, sending smaller compression pulses to catch up with the combustion front. This results in the in smaller velocity fluctuations that are observed. 


\subsection{Methane-Air}

Although the energetics and the laminar burning velocity for methane-air mixtures are similar to those for propane-air mixtures, methane is much less sensitive to detonation since its chemical induction time (at the same shock temperature for auto-ignition) is much longer than the other fuels in the alkane group. For a stoichiometric mixture of methane-air, the detonation cell size is also much larger compared to the tube diameter $(\lambda=32.0 \mathrm{~cm}$ for $\mathrm{D} / \lambda=0.875) .{ }^{24}$ Thus, according to the $\mathrm{D} / \lambda \approx 1$ criterion, methane-air is outside the limit where quasi-detonations can be observed. It is, therefore, surprising that supersonic combustion waves with an averaged velocity around $1000 \mathrm{~m} / \mathrm{s}$ can still be observed for a stoichiometric mixture of methane-air as shown in Fig. 32. The supersonic velocity at which the combustion front propagates confirms that it is driven by the rapid gasdynamic expansion of the products since the energetics of methane-air are similar to those of propane-air.

The high speed turbulent deflagrations for methane-air mixtures are found to exhibit significant velocity fluctuations with amplitudes as large as $1000 \mathrm{~m} / \mathrm{s}$ for the entire range of $0.8<\phi<1.2$ (see Fig. 33). In comparison to previous results for a circular tube, the averaged velocities obtained in the present study are consistently greater, yet span a more narrow range of equivalence ratios. The fluctuations in the local velocity exhibit irregular periods of oscillation, indicating that the fluctuations do not arise from perturbations generated by regularly spaced obstacles. Instead, the local quenching and the subsequent re-ignition of unburned gas pockets downstream is more likely to occur due to the slow kinetics of methane-air mixtures. As is the case with ethylene-air and propane-air mixtures near the lean transition limit, greater velocities approaching the C-J detonation velocity are observed when the blockage ratio is reduced from 0.41 to 0.19 (Fig. 34). The transition limits, however, remain unaffected with the change in blockage ratio.

\subsubsection{Sensitization of methane-air with $5 \%$ ethane}

Since methane-air is much less sensitive to shock induced auto-ignition than propane-air due to its long induction time, the fact that it can support the propagation of 
supersonic combustion waves can not be explained in terms of shock-induced auto-ignition. This is evident in experiments that were carried out by sensitizing methane-air to shock-induced auto-ignition with ethane. In general, a methane $\left(\mathrm{CH}_{4}\right)$ molecule is very stable and requires high activation energy to break the $\mathrm{C}-\mathrm{H}$ bond since the $\mathrm{H}$ atoms are bound tightly to the $\mathrm{C}$ atom. Once an $\mathrm{H}$ atom is removed, the methyl $\left(\mathrm{CH}_{3}\right)$ radical may not be oxidized directly but may recombine with another $\mathrm{CH}_{3}$ radical to form ethane $\left(\mathrm{C}_{2} \mathrm{H}_{6}\right)$. It is much easier to remove $\mathrm{H}$ atoms from ethane than it is from methane; therefore, due to the presence of $\mathrm{C}_{2} \mathrm{H}_{6}$, there is a larger quantity of $\mathrm{H}$ radicals that is available to initiate the chain branching reaction. It has been shown by Westbrook and Haselman ${ }^{25}$ that the induction time can decrease by factor of two when $5 \%$ ethane is introduced into methane. Therefore, in the present investigation, a stoichiometric mixture was used where the fuel was comprised of $5 \%$ ethane:

$$
\left(0.95 \mathrm{CH}_{4}+0.05 \mathrm{C}_{2} \mathrm{H}_{6}\right)+2.075\left(\mathrm{O}_{2}+3.76 \mathrm{~N}_{2}\right) \rightarrow 1.05 \mathrm{CO}_{2}+2.05 \mathrm{H}_{2} \mathrm{O}+7.8 \mathrm{~N}_{2} \text {. }
$$

The energetics of the mixture was not altered by this small addition of ethane. Using, a thermodynamic equilibrium code (STANJAN), it was found that the adiabatic flame temperature did not change significantly from $2213 \mathrm{~K}$ to $2216 \mathrm{~K}$. There was no observable effect on the high speed turbulent deflagration velocities due to the addition of ethane (Fig. 35). Without a change in the propagation velocity for methane-air mixtures sensitized with ethane, auto-ignition of high speed turbulent deflagrations is not effected by shock compression but rather by turbulent mixing due to the obstacles.

\subsubsection{The importance of transverse shocks}

To demonstrate the necessity of the transverse shocks in the turbulent mixing process of high speed turbulent deflagrations, experiments were also carried out where the transverse shocks are dampened upon reflection from obstacles wrapped with rope (used as an acoustically absorbing material). In order to facilitate comparisons, the smaller obstacles $(B R=0.19)$ were used in these experiments. Once wrapped with the acoustically absorbing material, the blockage ratio would be equivalent to that of the large obstacle array $(B R=0.41)$. It can be seen in Fig. 36 that there is a decrease of $200 \mathrm{~m} / \mathrm{s}$ in the propagation velocity for stoichiometric mixture of methane-air when transverse waves are attenuated. No attempt was made to line the walls of the tube with 
acoustic absorbing material. A greater effect may have been observed if the transverse shocks reflected from the tube walls were eliminated. This result is similar to that found by Teodorcyk and Lee ${ }^{26}$ where a quasi-detonation, propagating through an obstacle-laden tube lined with acoustically absorbing material, fails and is reduced to a turbulent deflagration as the transverse shocks become attenuated. Essentially, the role of transverse shock waves is to maintain the intense turbulence in the reaction zone through the production of shear layers and of vorticity. In Fig. 37, it can be seen that a shock propagating through obstacles similar to those in the present investigation is scattered and produces a field of transverse waves. ${ }^{27}$ As well, vortex structures are generated by the shock near the obstacles. The shock-shock, shock-vortex, and vortex-vortex interactions facilitate the viscous dissipation of energy, which results in the high propagation velocities that are observed.

Experiments were also conducted where the obstacles in the last half of the tube are removed. It is found that, for a stoichiometric methane-air mixture, the high supersonic velocity can not be maintained as shown in Fig. 38. The deflagration is found to decay to velocities lower than $400 \mathrm{~m} / \mathrm{s}$ by the end of the tube. It appears that the velocity will decay even further provided an even longer distance of flame travel were available. Thus, it is clear that without the ensemble of interacting transverse shocks, the rapid turbulent mixing responsible for auto-ignition of these supersonic high speed turbulent deflagrations can not be sustained. 


\section{Chapter 4 Concluding remarks}

On the basis of the present experimental results, it may be concluded that the propagation mechanisms of all supersonic combustion waves (C-J detonations, quasi-detonations, and high speed turbulent deflagrations) are similar. The reaction zones of all supersonic combustion waves are driven by the expansion of the high pressure combustion products at the constant volume explosion pressure. Auto-ignition is achieved by a combination of both adiabatic compression and obstacle enhanced turbulent mixing. It is only the degree which shock heating or turbulent mixing controls the auto-ignition process that differs amongst the different regimes of supersonic combustion waves. For C-J detonations and quasi-detonations where shock temperatures are sufficiently high, auto-ignition due to adiabatic shock compression dominates. For high speed turbulent deflagrations where the shock temperatures are below the auto-ignition limit, shock heating alone is no longer sufficient. Instead, auto-ignition is augmented by rapid turbulent mixing, which is effectively enhanced by the obstacles that permit numerous vorticity production mechanisms. There is, however, still a certain degree of localized temperature rise due to compression across the transverse waves that leads to auto-ignition. Moreover, there is a further temperature increase associated with adiabatic compression across the reaction zone to the constant volume explosion pressure. Even methane-air mixtures (which are extremely insensitive to detonation on the basis of shock induced auto-ignition) can support supersonic high speed turbulent deflagrations in rough-walled or obstacle-filled tubes through the rapid turbulent mixing mechanism.

It was also found that rapid turbulent mixing from the obstacles can quench a propagating flame in lean near-limit mixtures where the induction kinetics become slow. When quenching occurs, the pressure rise across the combustion front decreases due to the decrease in energy release. Therefore, the work done by the gasdynamic expansion to drive the flame is also decreased, and it becomes no longer possible to sustain supersonic wave propagation. As quenched pockets of unburned mixture are swept downstream and 
explode at a later time, pressure pulses are sent forward and reach the combustion front, thereby accelerating it and causing the observed pulsations in the local velocity.

As well, the previously reported "choking" regime was not observed in the present investigation. It may, therefore, be concluded that this particular regime is unique to only the orifice plate obstacle geometry. The flame propagates as a consequence of the sequential venting of the combustion products through choked orifices and subsequently travels at the local sound speed of the combustion products. For this reason, the "choking" regime can not be considered to be an actual propagation regime since the mechanism is controlled by gasdynamic jetting and not by turbulent combustion.

\subsection{Future work}

From the perspective of computer modeling of accidental industrial explosions, the sub-models for turbulence and for combustion must be modified to realistically describe the propagation mechanisms of supersonic combustion waves. The turbulence models should account for vorticity production and for energy dissipation by shock waves. Auto-ignition mechanisms via adiabatic shock compression and turbulent mixing need to be incorporated into the combustion sub-models to accurately describe the propagation of quasi-detonations and of high speed turbulent deflagrations.

Experimentally, a more detailed study of steady state propagation velocities through local velocity fluctuations has only begun in the effort to elucidate the propagation mechanisms of supersonic combustion waves. Presently, the current study is limited to time of arrival and pressure measurements. It would of interest to visualize the structure of the supersonic combustion waves through high speed Schlieren photography. Perhaps this will lead to more insight into the degree and under what conditions turbulent mixing results in auto-ignition. These experiments would need to be conducted at low pressures due to the high over-pressures developed in atmospheric fuel-air mixtures. Thus, it would also be of interest to see if the transition limit criterion of $D / \lambda \approx 1$ is independent of initial and boundary conditions. In other words, if the tube and obstacle dimensions were scaled differently (with the same aspect ratios) or if the initial pressure of the mixture were other than atmospheric, would the transition criterion of $D / \lambda \approx 1$ still hold? 
There is also the question of the effect of the Lewis number on turbulent burning velocities and local velocity fluctuations. The Lewis number is defined as the ratio of heat diffusion to mass diffusion of the deficient reactant. In the present investigation, the propagation velocities observed are far too high for ignition to be controlled by mechanisms of diffusion and transport. However, it may be necessary to investigate the effect of the Lewis number (if any) as quenched pockets of unburned mixture react downstream or on the micro-scale of the turbulent eddies within the reaction zone of high speed turbulent deflagrations.

Ultimately, the aim is to seek a fundamental quantitative understanding of turbulent flame acceleration by obstacles. Based on the wealth of experimental data accumulated over the years for different fuel-oxidizer mixtures, tube dimensions, and obstacle configurations, the goal is to formulate a universal correlation of the propagation velocities with fluid mechanic parameters rather than with characteristic geometric parameters of the experimental apparatus. 


\section{References}

1. Chapman, W.R., and R.V. Wheeler, "The Propagation of Flame in Mixtures of Methane and Air. Part IV. The Effect of Restrictions in the Path of the Flame," Journal of the Chemical Society, 37:2139-2147, 1926.

2. Laffitte, P., "Sur la formation de l'onde explosive," Académie des Sciences, 176:1392-1395, 1923.

3. Shchelkin, K.I., "Effect of Roughness of the Surface in a Tube on Origination and Propagation of Detonation in Gases," Journal of Experimental and Theoretical Physics, 10:823-827, 1940.

4. Guénoche, H., and N. Manson, "Influence des conditions aux limites transversales sur la propagation des ondes de choc et de combustion," Revue de l'Insitut Français du Pétrole, 2:53-69, 1949.

5. Zel'dovich, Ya. B., A.A. Borisov, B.E. Gel'fand, S.V. Chomiak, and A.E. Mailkov, "Low Speed Quasi-Detonation Regimes of Combustion of Fuel-Air Mixtures in Rough Tubes," Soviet Phys.-Doklady, 27:1360-1362, 1984.

6. Popat, N.R., C.A. Caitlin, B.J. Arntzen, R.P. Lindstedt, B.H. Hjertager, T. Solberg, O. Saeter, and A.C. Van den Berg, "Investigations to Improve and Assess the Accuracy of Computational Fluid Dynamic Based Explosion Models," Journal of Hazardous Materials, 45:1-25, 1996.

7. Lee, J.H.S., "Mechanisms of High-Speed Deflagrations and Quasi-Detonations and their Numerical Modeling," Presented at the $3^{\text {rd }}$ International Seminar of Fire and Explosion Hazard, Lake Windermere, U.K., April 10-14, 2000.

8. Lee, J.H., R. Knystautas, and C.K. Chan, "Turbulent Flame Propagation in Obstacle-Filled Tubes," Proceedings of the Combustion Institute, 20:1663-1672, 1984. 
9. Lee, J.H.S., "Fast Flames and Detonations," in T.M. Sloane (ed.) The Chemistry of Combustion Processes. American Chemical Society, 119-150, 1984.

10. Makris, A., "The Propagation of Gaseous Detonations in Porous Media," Ph.D. Thesis, McGill University, Montreal, Canada, 1993.

11. Lyamin, G.A., V.V. Mitrofanov, A.V. Pinaev, and V.A. Subbotin, "Propagation of Gas Explosion in Channels with Uneven Walls and in Porous Media," in A.A. Borisov (ed.) Dynamic Structure of Detonation in Gaseous and Dispersed Media. 51$75,1991$.

12. Lee, J.J., G. Dupré, R. Knystautas, and J.H. Lee, "Doppler Interferometry Study of Unstable Detonations," Shock Waves, 5:175-181, 1995.

13. Teodorcyk, A., J.H.S. Lee, and R. Knystautas, "Propagation Mechanism of Quasi-Detonations," Proceedings of the Combustion Institute, 22:1723-1731, 1988.

14. Teodorcyk, A., J.H.S. Lee, and R. Knystautas, "The Structure of Fast Turbulent Flames in Very Rough, Obstacle-Filled Channels," Proceedings of the Combustion Institute, 23:735-741, 1990.

15. Atkinson, R., D.C. Bull, and P.J. Shuff, "Initiation of Spherical Detonation in Hydrogen/Air," Combustion and Flame, 39:287-300, 1980.

16. Shultz, E., and J. Shepherd, "Validation of Detailed Reaction Mechanism for Detonation Simulation," Explosion Dynamics Laboratory Report FM99-5, California Institute of Technology, 2000.

17. Westbrook, C.K., and P.A. Urtiew, "Chemical Kinetic Prediction of Critical Parameters in Gaseous Detonations," Proceedings of the Combustion Institute, $19: 615-623,1982$.

18. Knystautas R., J.H. Lee, O. Peraldi, and C. Chan, "Transition of a Flame from a Rough to a Smooth-Walled Tube," in J.R. Bowen, J.-C. Leyer, and R.I. Soloukhin (eds.) Dynamics of Explosions. AIAA Progress in Astronautics and Aeronautics, $106: 37-52,1985$. 
19. Ashurst, W.T., and P.K. Barr, "Discrete Vortex Simulation of Flame Acceleration due to Obstacle Generated Flow," Sandia National Laboratory (Livermore) Report SAND 82-8724, 1982.

20. Kuznetsov, M.S., I.D. Matsukov, V.I. Alekseev, and S.B. Dorofiev, "Photographic Study of Unstable Turbulent Flames in Obstructed Channels," Proceedings of the $17^{\text {th }}$ ICDERS, Heidelberg, Germany, July 25-30, 1999.

21. Thibault, P., Y.K. Liu, C. Chan, J.H. Lee, R. Knystautas, C. Guirao, B. Hjertager, and K. Fuhre, "Transmission of an Explosion Through an Orifice," Proceedings of the Combustion Institute, 19:599-606, 1982.

22. Lieberman, D.H.B., and J.H.S. Lee, "Photographic Study of the Transition Between the Quasi-Detonation and Choking regimes," Proceedings of the $18^{\text {th }}$ ICDERS, Seattle, USA, July 29-Aug. 3, 2001.

23. Carnasciali, F., J.H.S. Lee, R. Knystautas, and F. Fineschi, "Turbulent Jet Initiation of Detonation," Combustion and Flame, 84:170-180, 1991.

24. Caltech Detonation Database at www.galcit.ca/tech.edu/detn_db/html/searcher.html.

25. Westbrook, C.K., and L.C. Haselman, "Chemical Kinetics in LNG Detonations," Proceedings of the $7^{\text {th }}$ ICOGER, Göttingen, Germany, AIAA, 193-206, 1980.

26. Teodorcyk, A., and J.H.S. Lee, "Detonation Attenuation by Foams and Wire Meshes Lining the Walls," Shock Waves, 4:225-236, 1995.

27. Lee, J.H.S., "Shock-Vortex Interaction: Its Role in Compressible Turbulence and Detonation Structure," Proceedings of the $2^{\text {nd }}$ International Workshop on Shock Wave/Vortex Interaction, Sendai, Japan, 1997. 


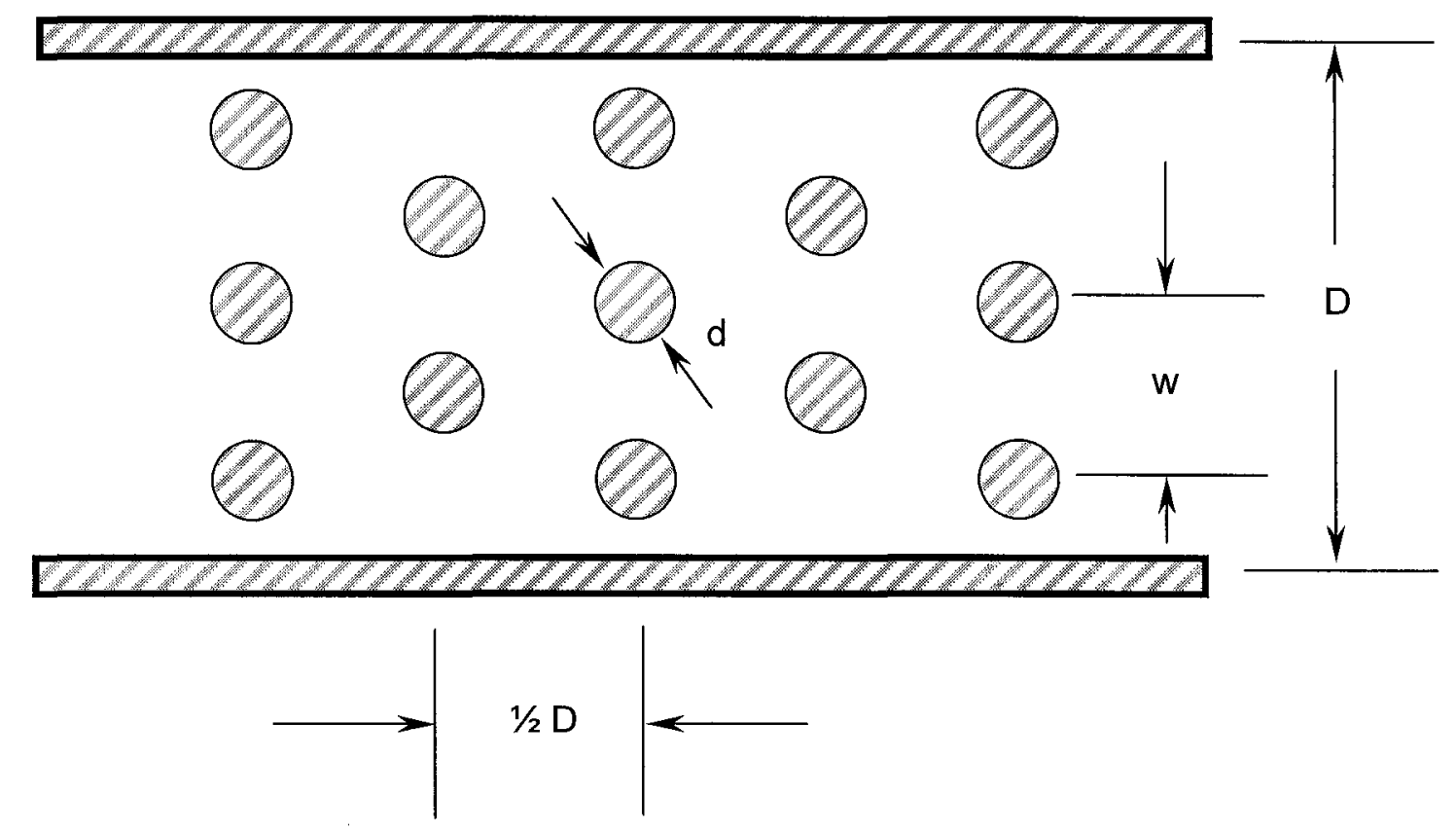

Fig. 1: Top view of tube-obstacle assembly. 


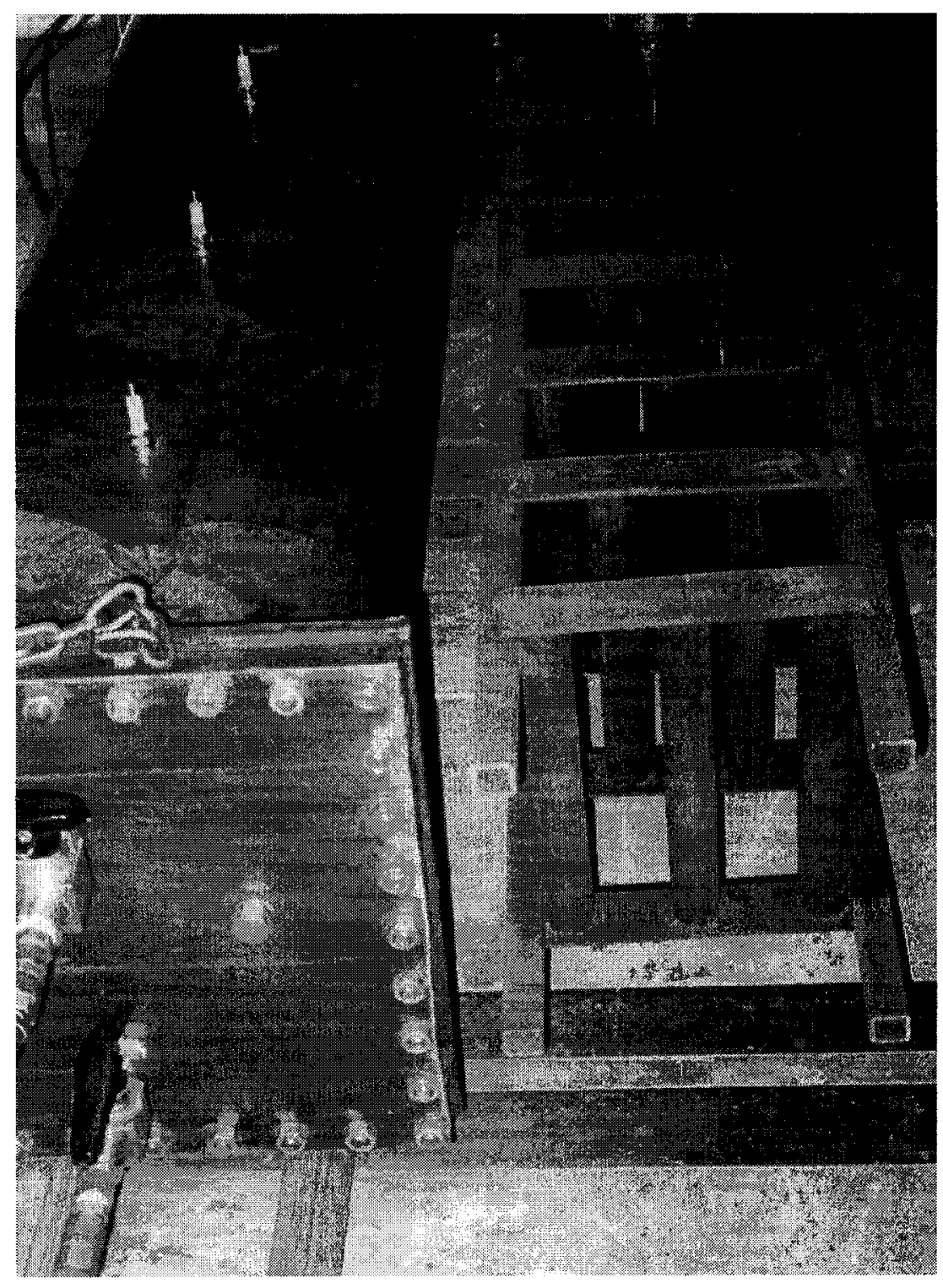

Fig. 2: Photograph of tube and obstacle array. 


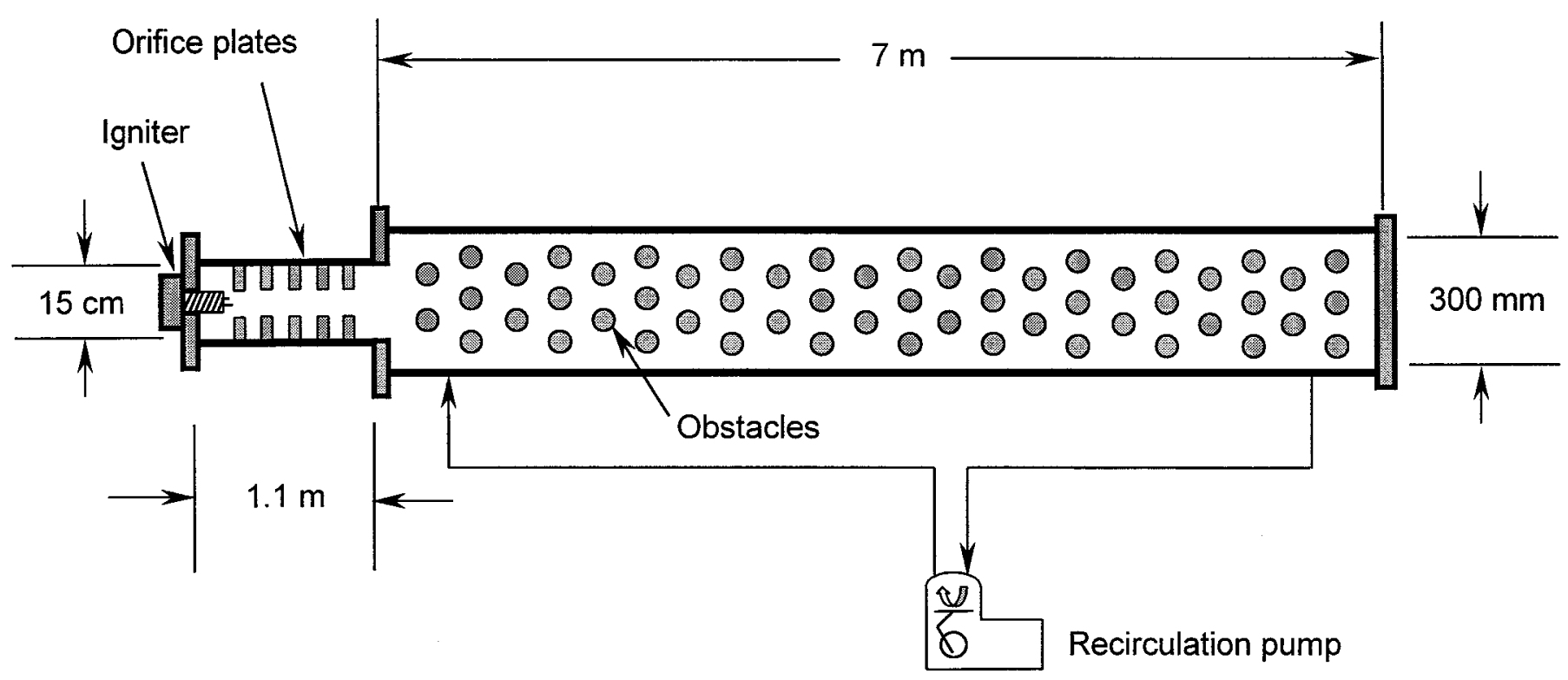

Fig. 3: Schematic of experimental set-up. 


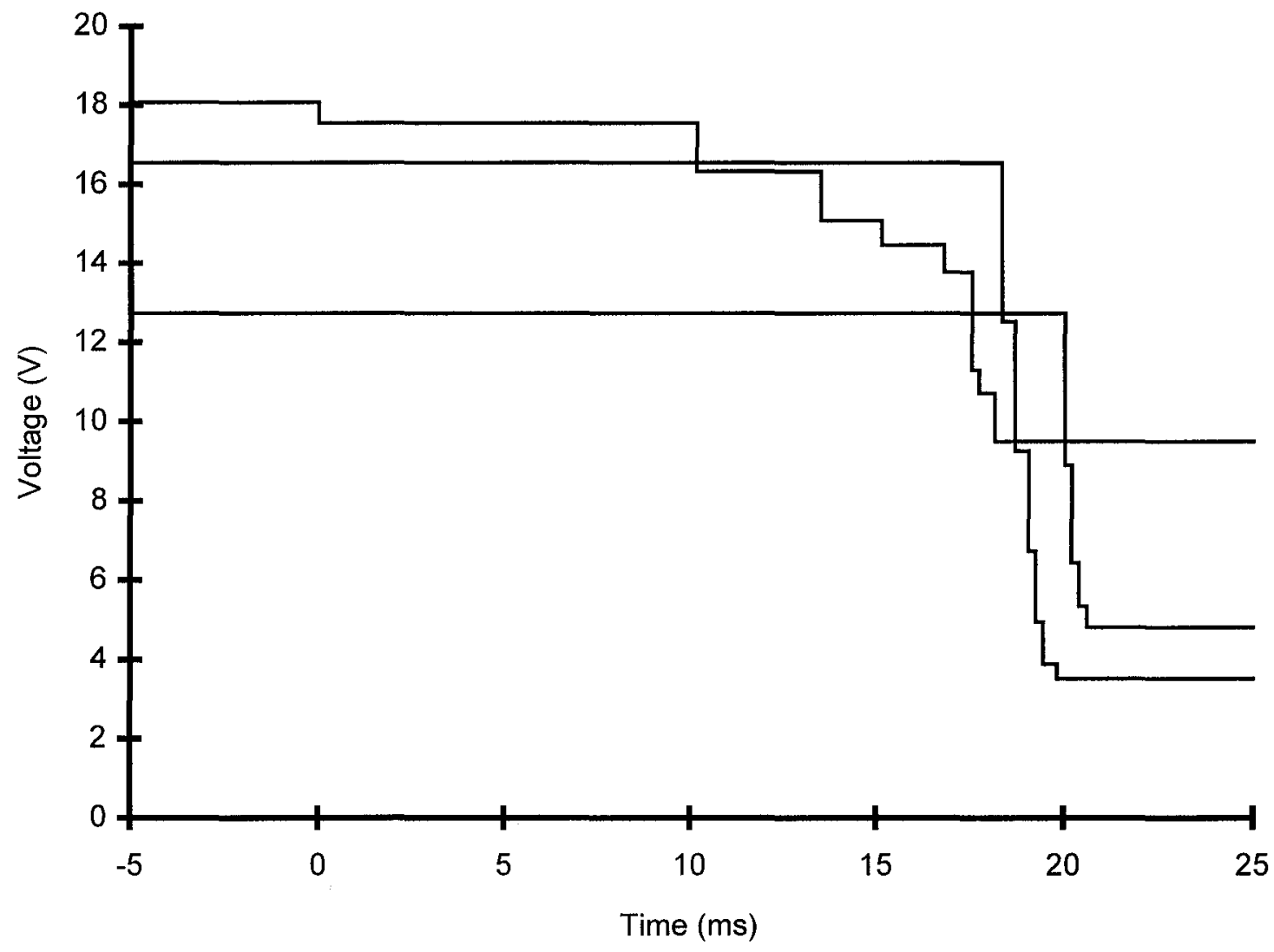

Fig. 4: Typical oscilloscope signals from ionization probes for stoichiometric propane-air $(\phi=1.0, \mathrm{BR}=\mathbf{0 . 4 1})$. 


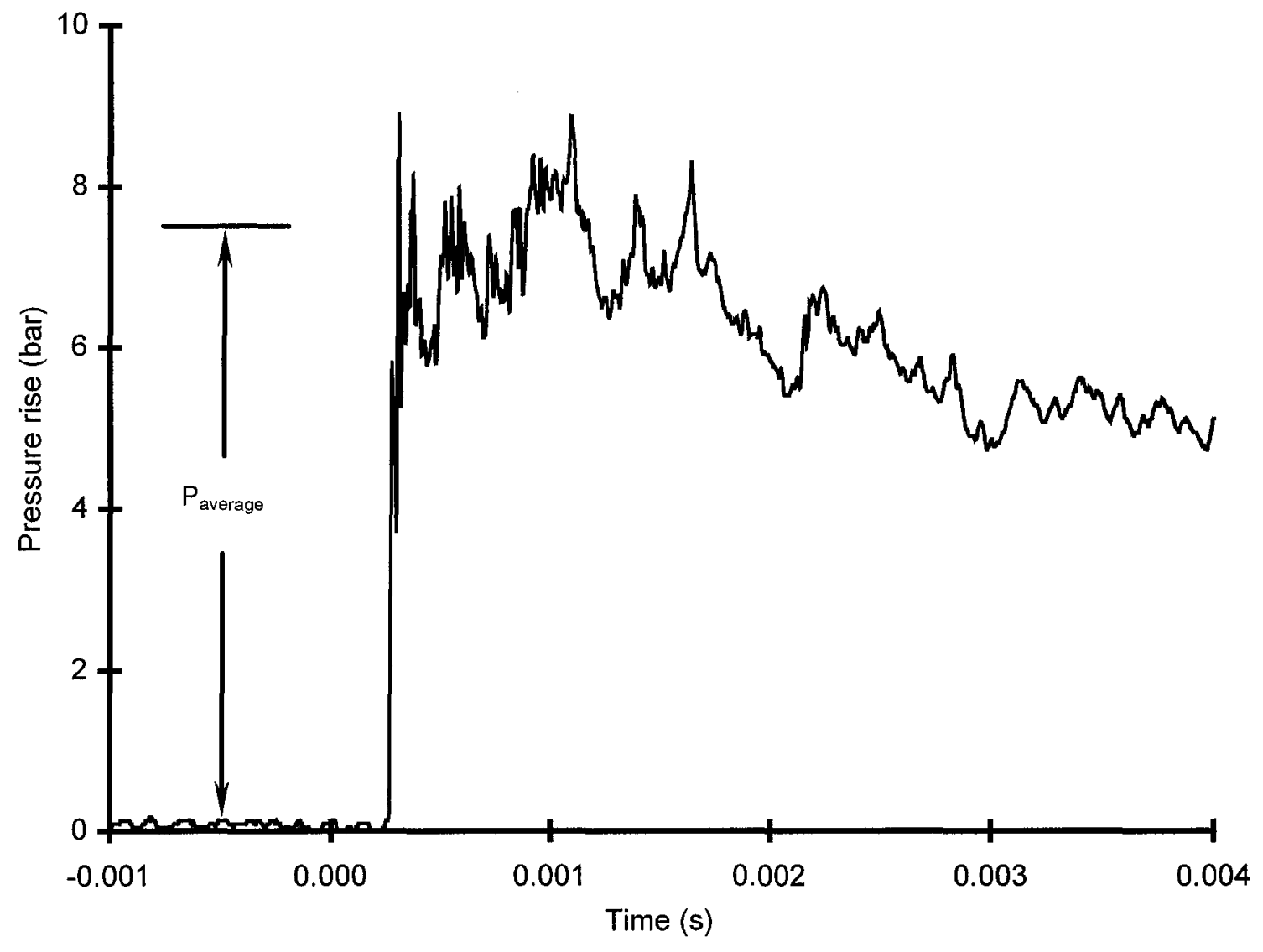

Fig. 5: Typical pressure profile of a high speed turbulent deflagration for stoichiometric methane-air $(\phi=1.0, B R=0.41)$. 


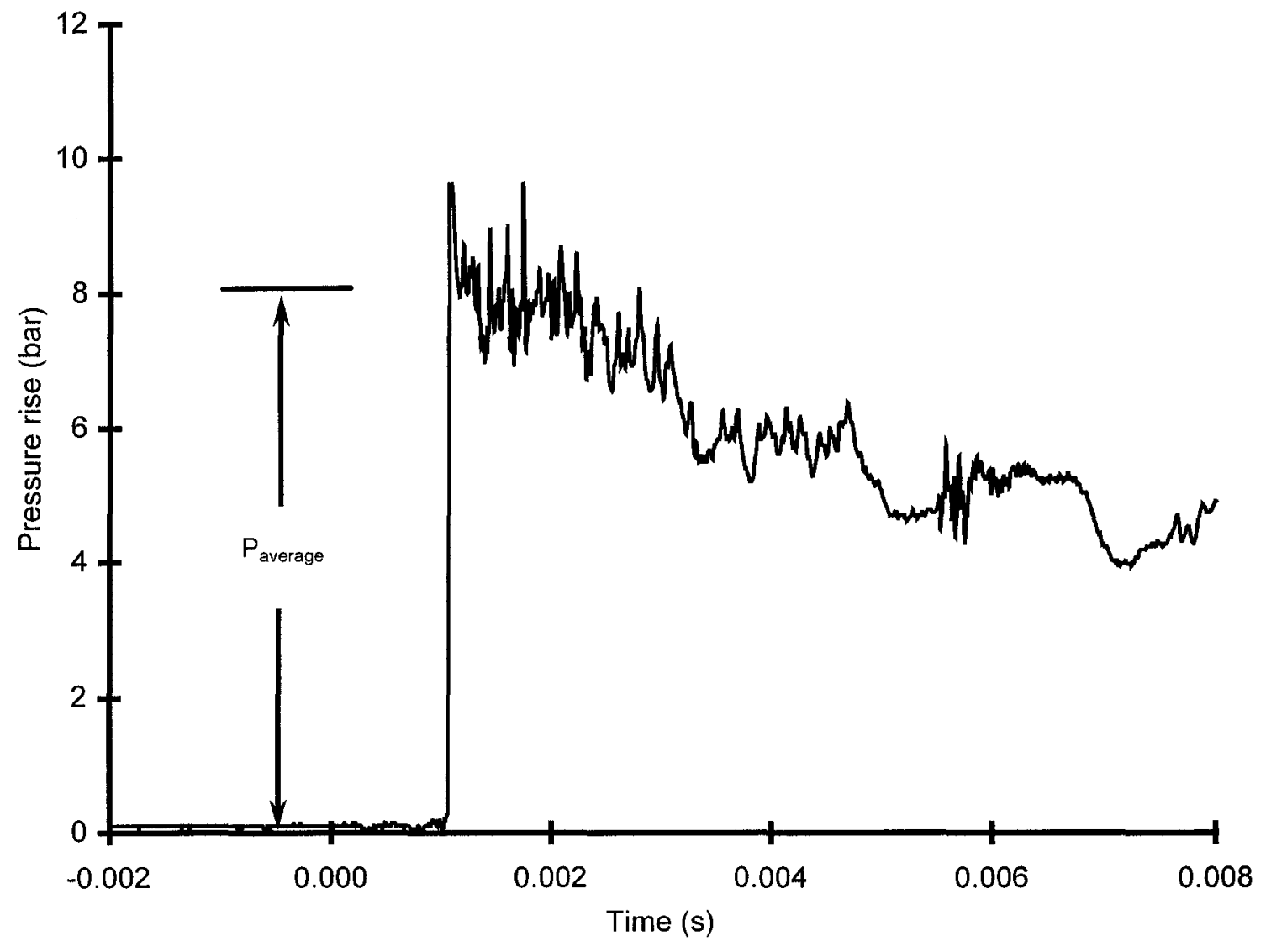

Fig. 6: Typical pressure profile of a quasi-detonation for stoichiometric propane-air $(\phi=1.0, \mathrm{BR}=0.41)$. 


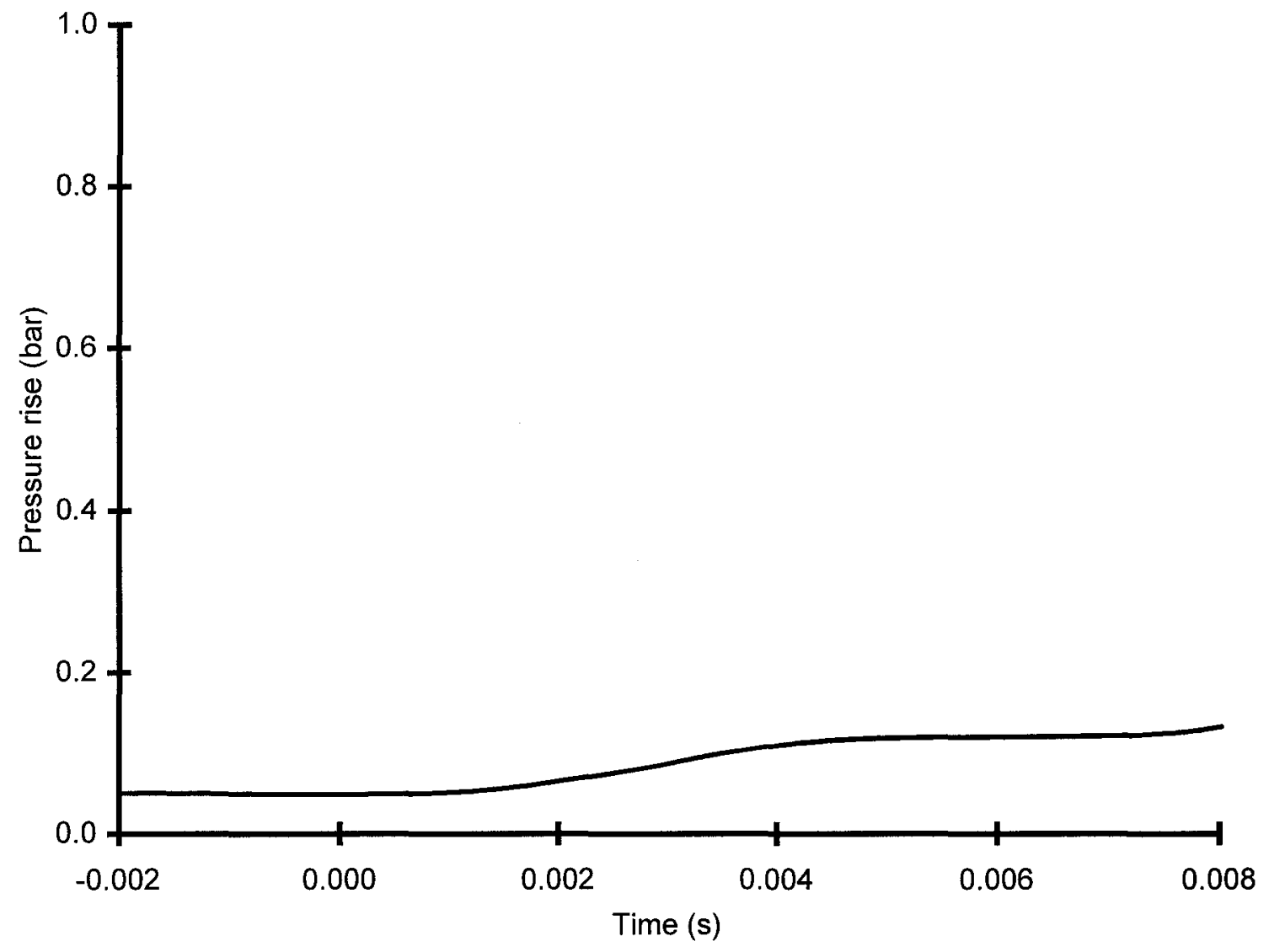

Fig. 7: Typical pressure profile of a slow turbulent deflagration for very lean propane-air $(\phi=0.55, \mathrm{BR}=\mathbf{0 . 4 1})$. 


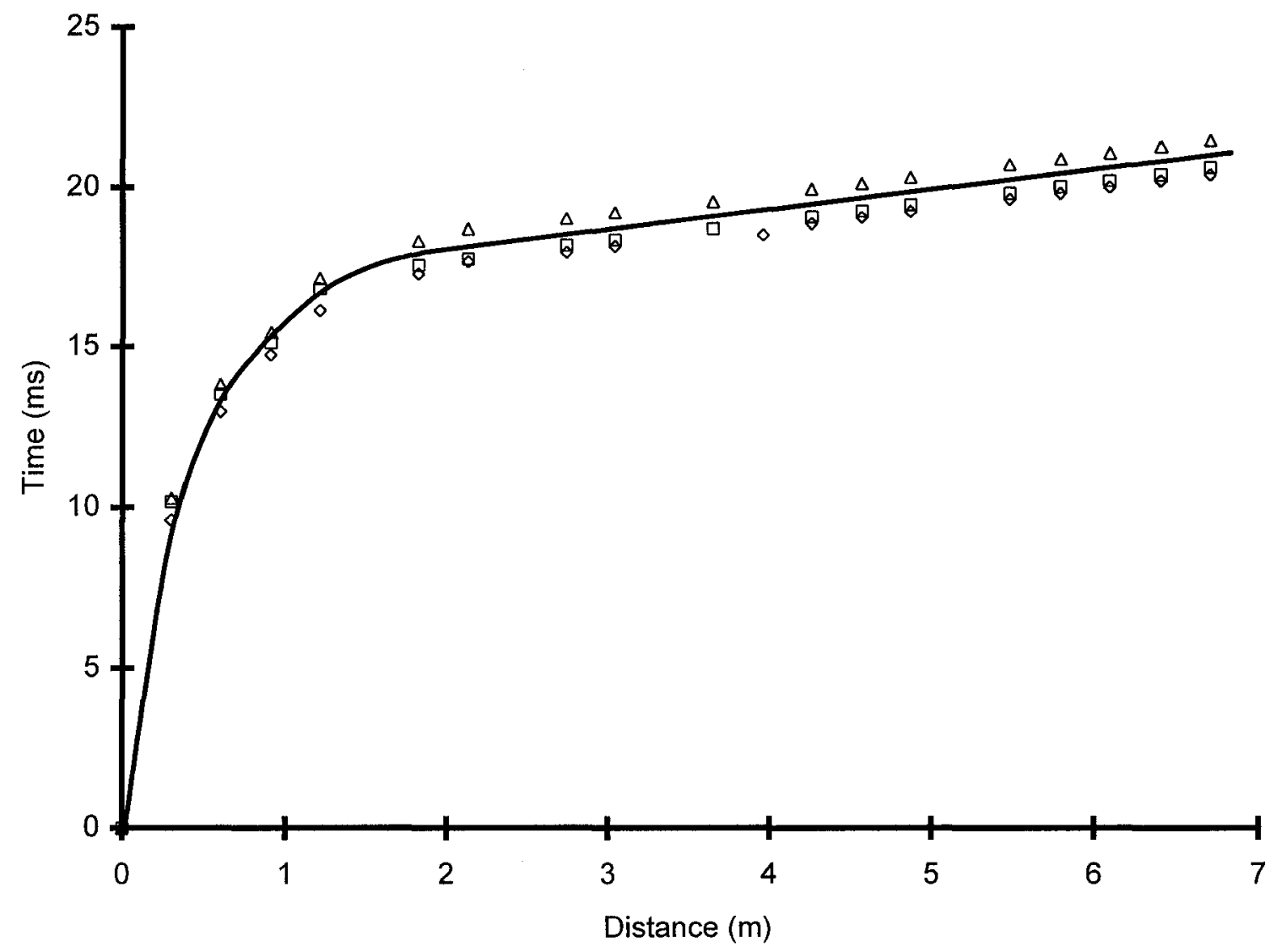

Fig. 8: Flame trajectory for stoichiometric propane-air $(\phi=1.0, \mathrm{BR}=0.41)$. 


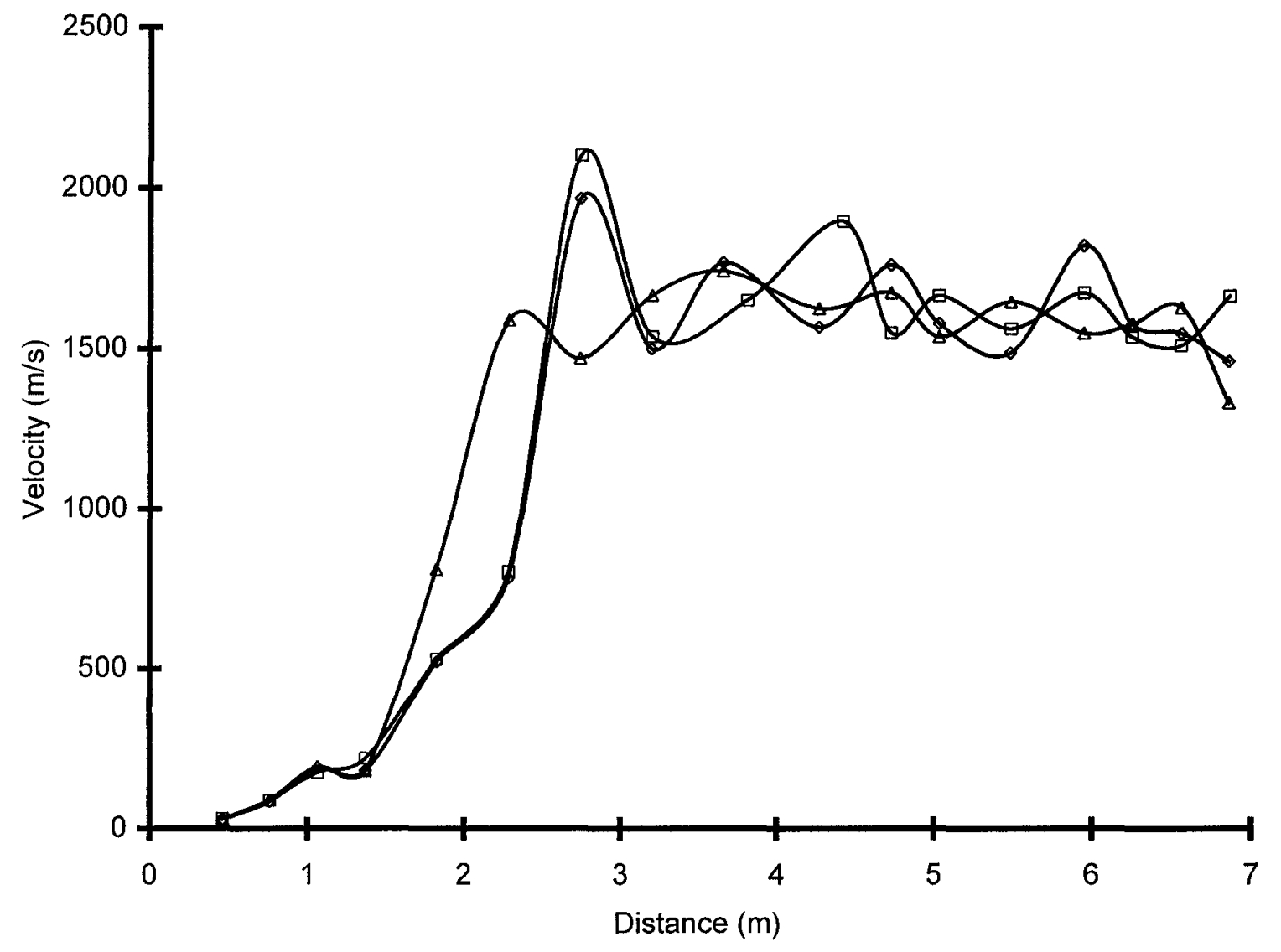

Fig. 9: Local velocity variation with distance along tube for stoichiometric propane-air $(\phi=1.0, B R=0.41)$. 


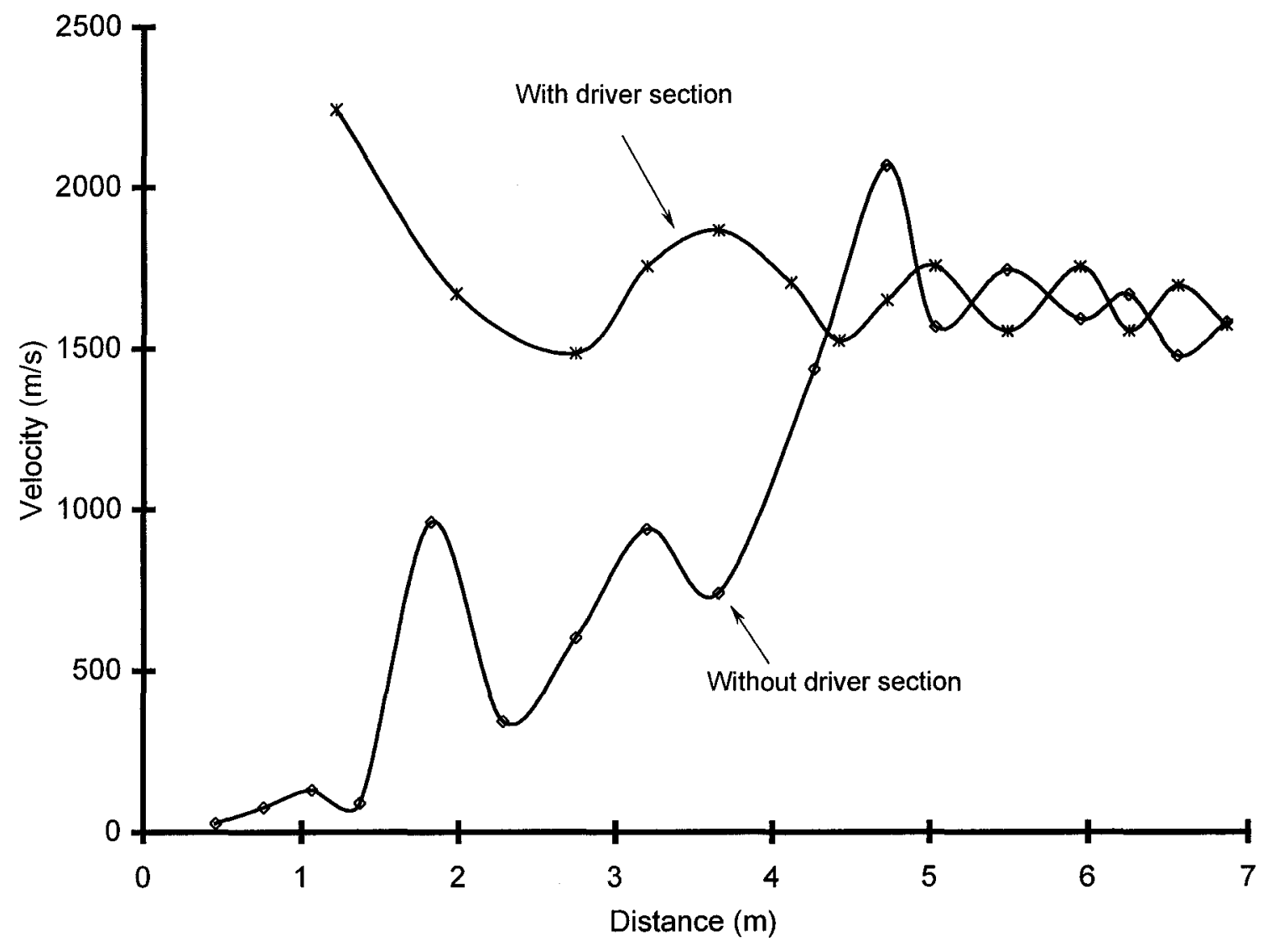

Fig. 10: Local velocity variation with distance along tube for rich propane-air $(\phi=1.4, B R=0.41)$ with and without driver section. 
a)

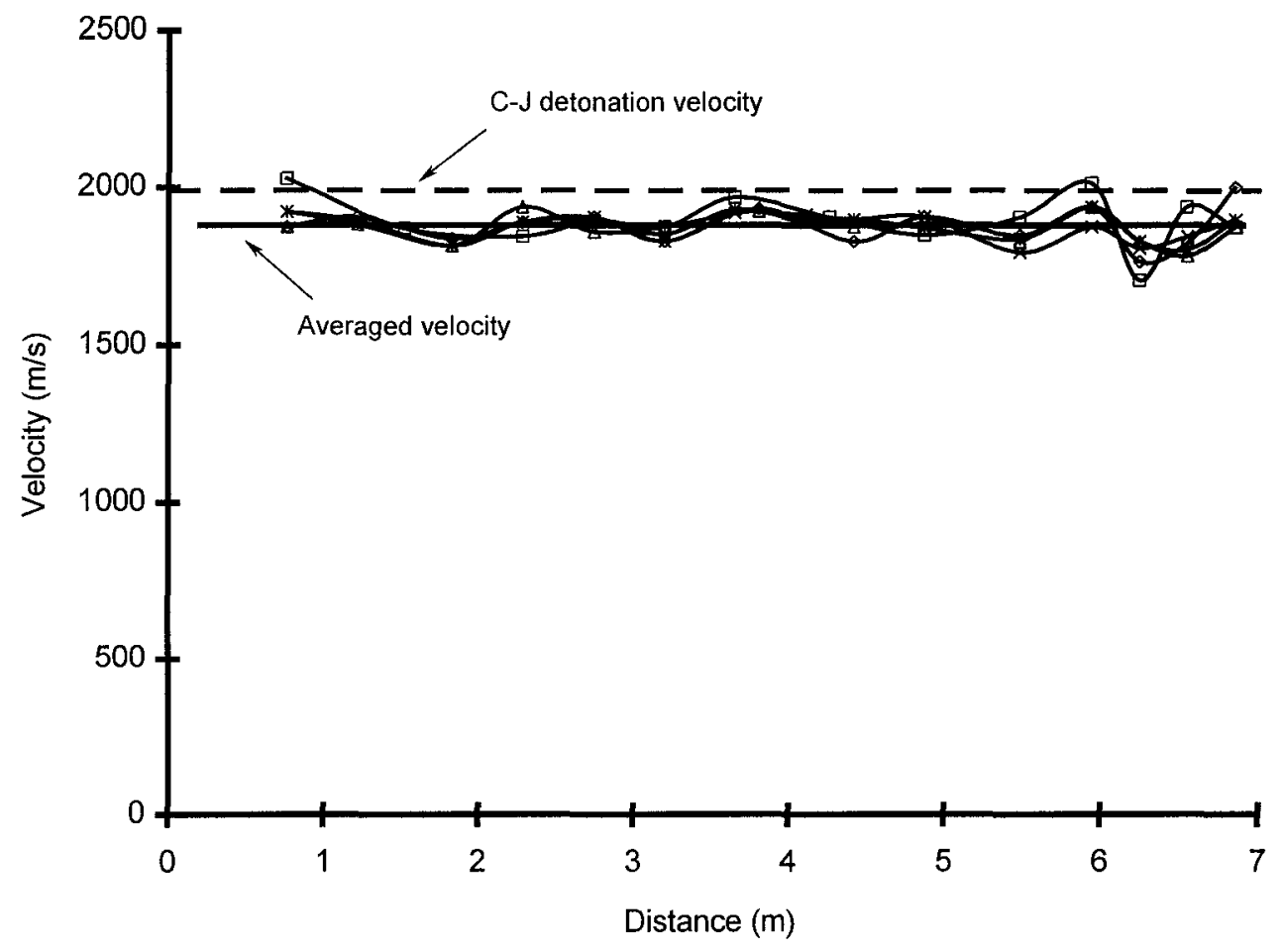

b)

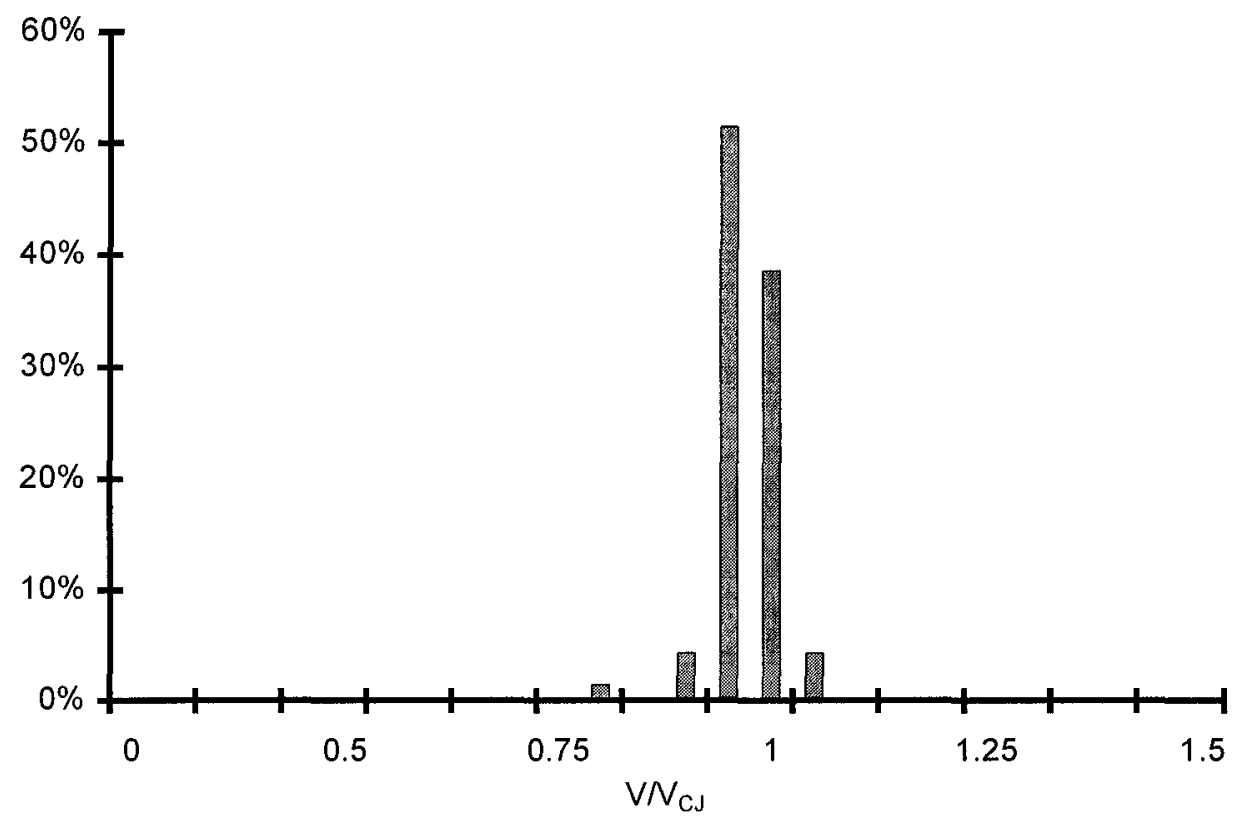

Fig. 11: a) Local velocity variation with distance along tube for stoichiometric hydrogen-air $(\phi=1.0, B R=0.41)$. b) Corresponding velocity histogram. 
a)

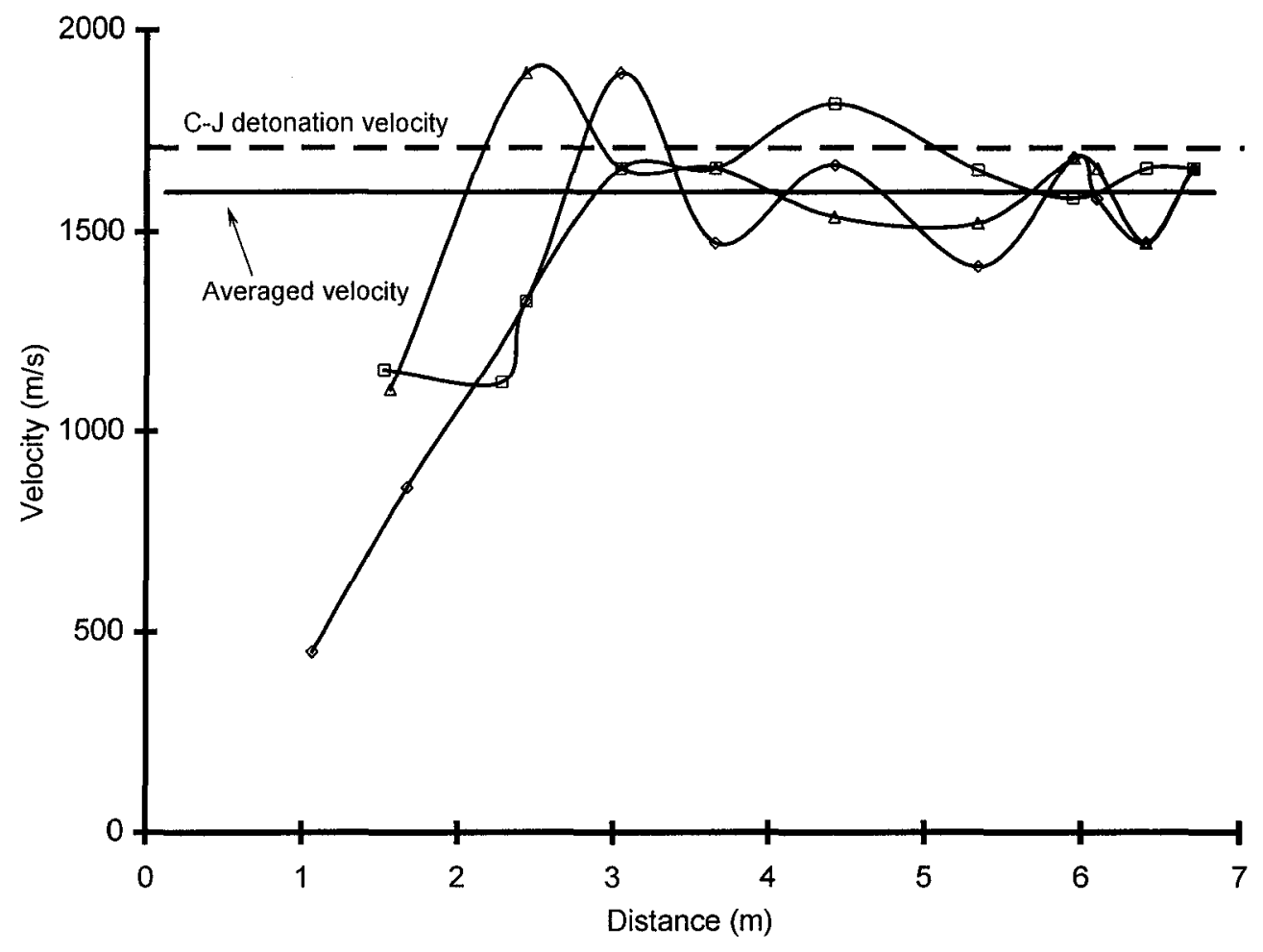

b)

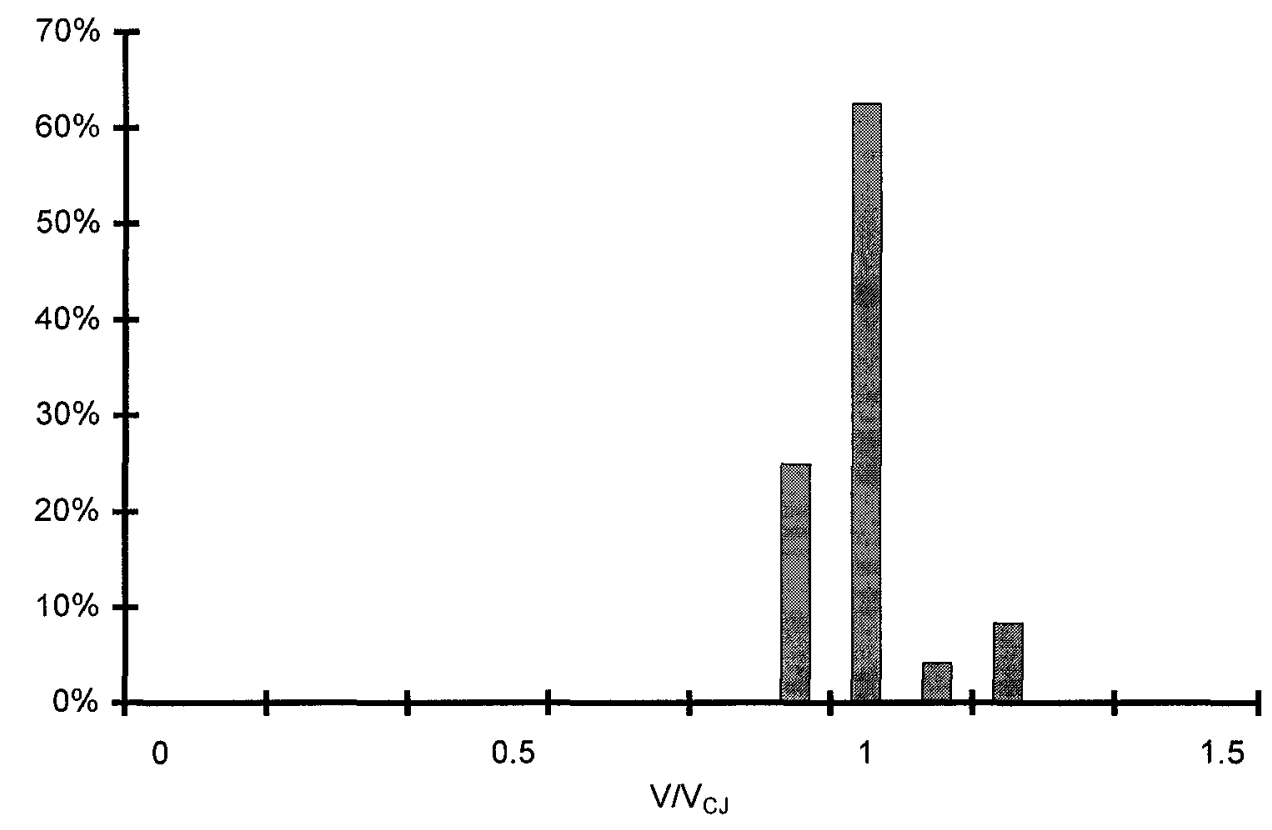

Fig. 12: a) Local velocity variation with distance along tube for hydrogen-air near lean limit of quasi-detonation regime $(\phi=0.6, \mathrm{BR}=0.41)$.

b) Corresponding velocity histogram. 
a)

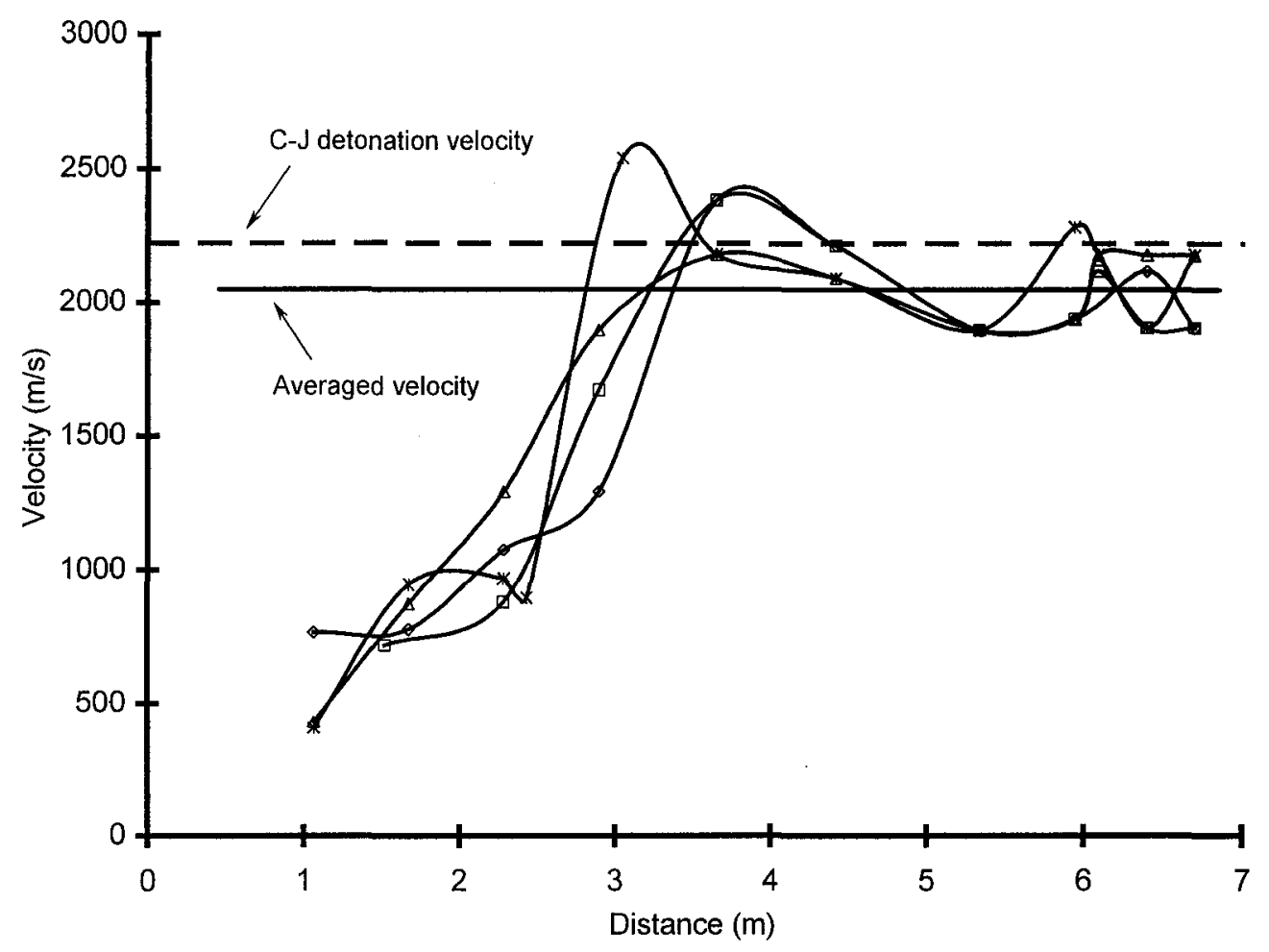

b)

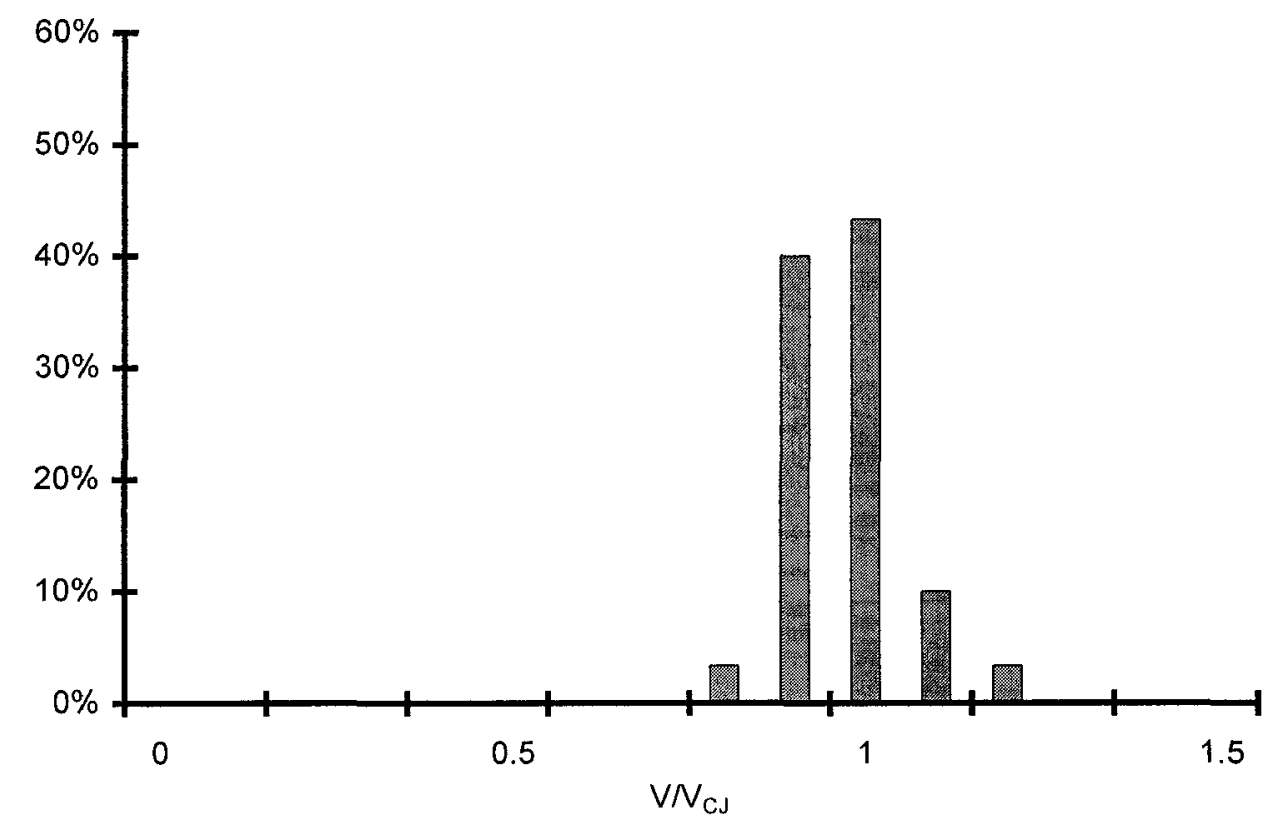

Fig. 13: a) Local velocity variation with distance along tube for hydrogen-air near rich limit of quasi-detonation regime $(\phi=3.1, \mathrm{BR}=0.41)$.

b) Corresponding velocity histogram. 
a)

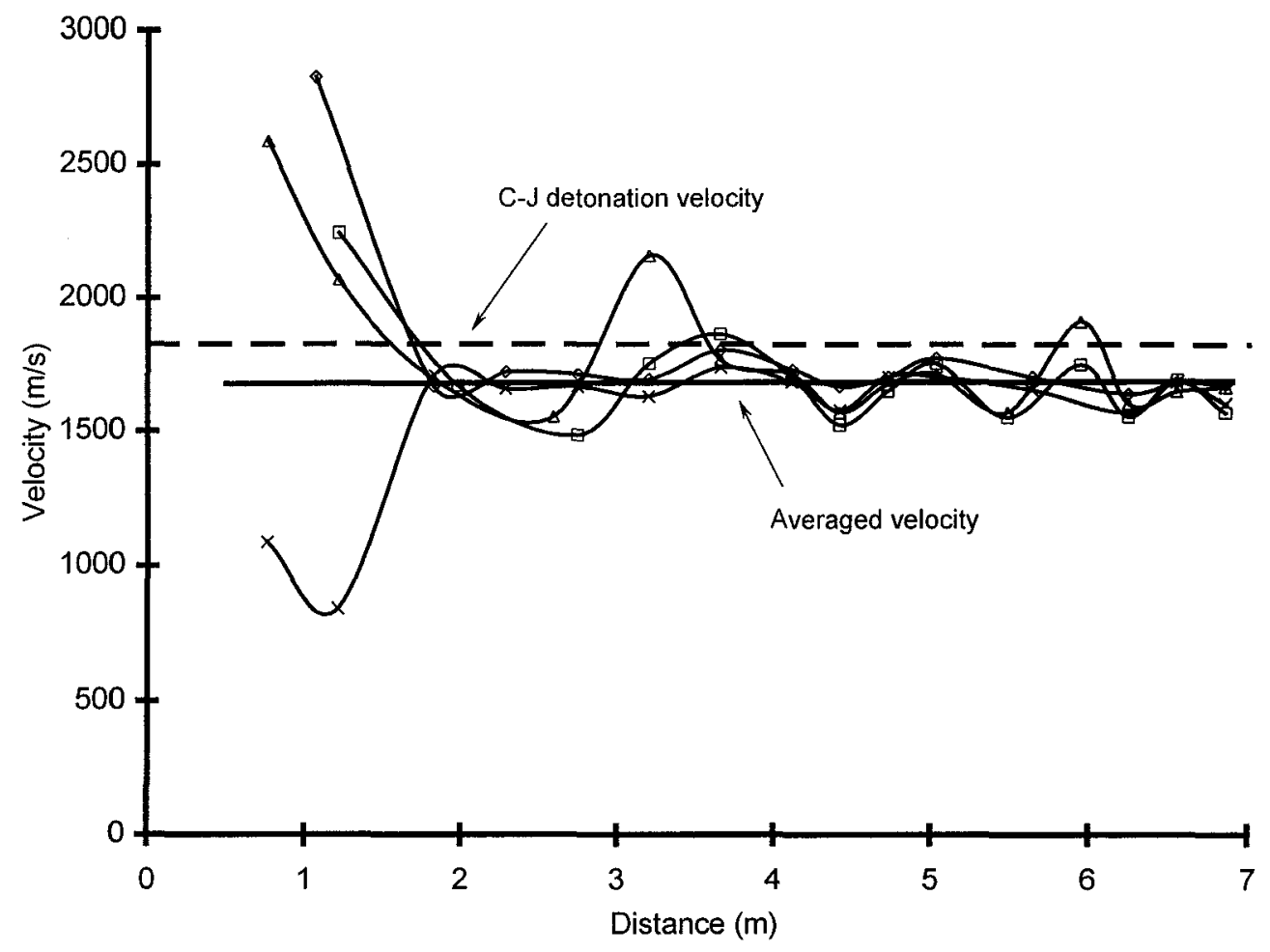

b)

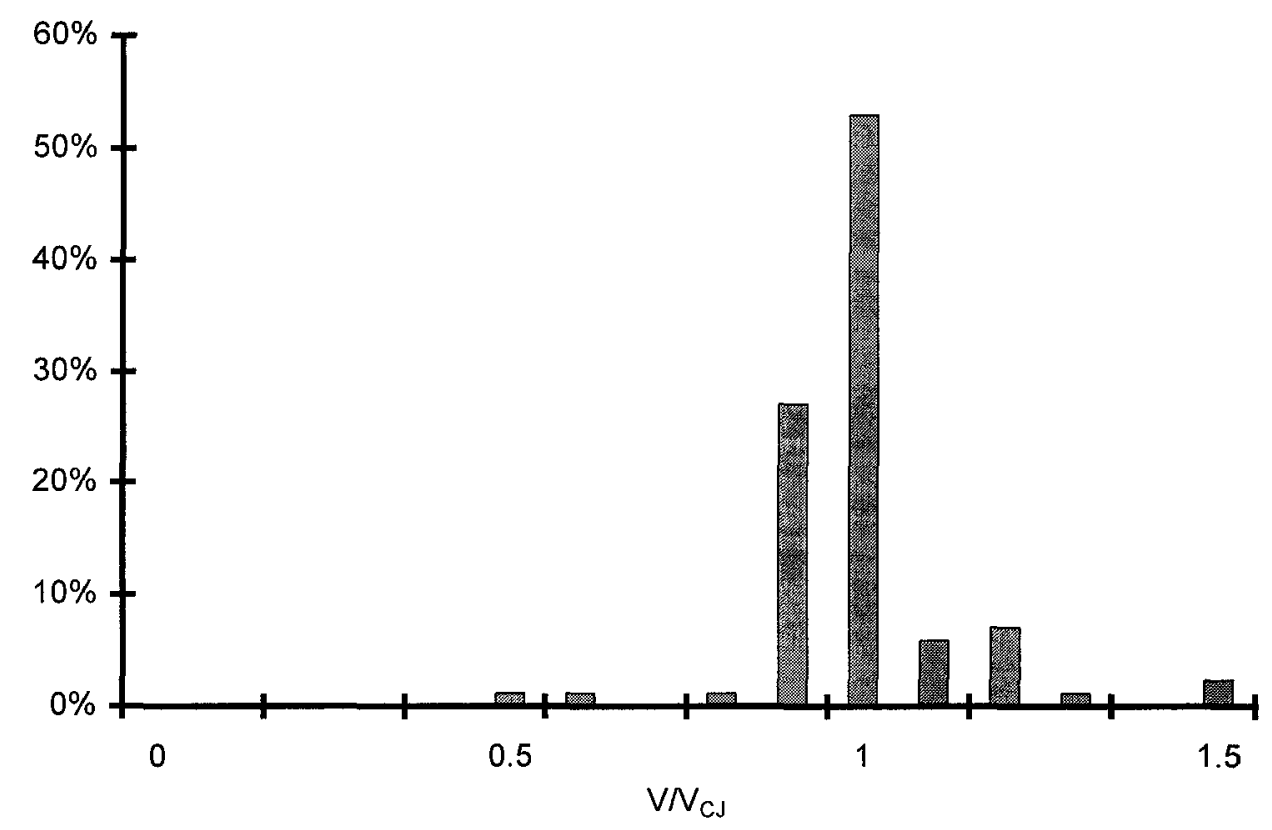

Fig. 14: a) Local velocity variation with distance along tube for stoichiometric ethylene-air $(\phi=1.0, \mathrm{BR}=0.41)$. b) Corresponding velocity histogram. 
a)

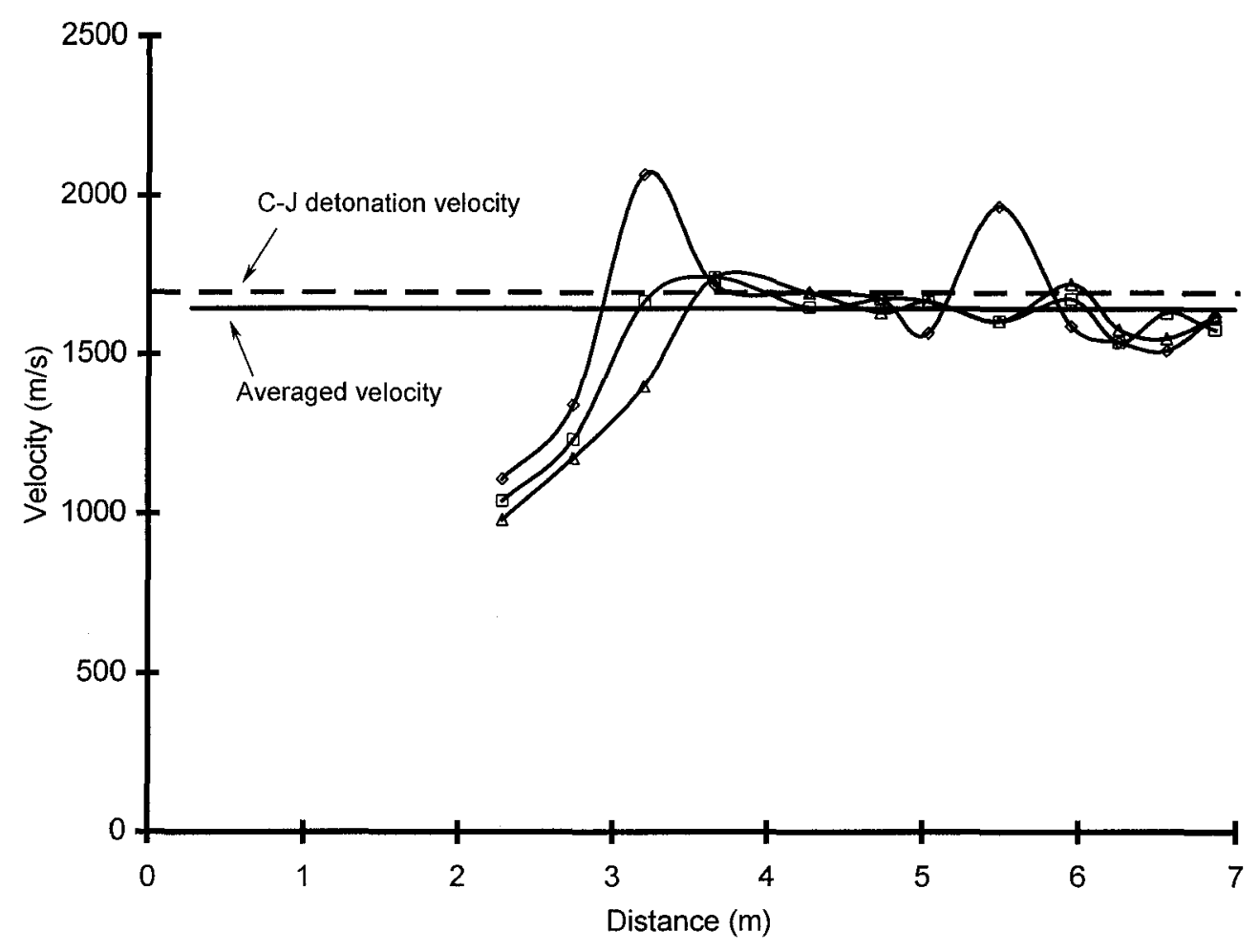

b)

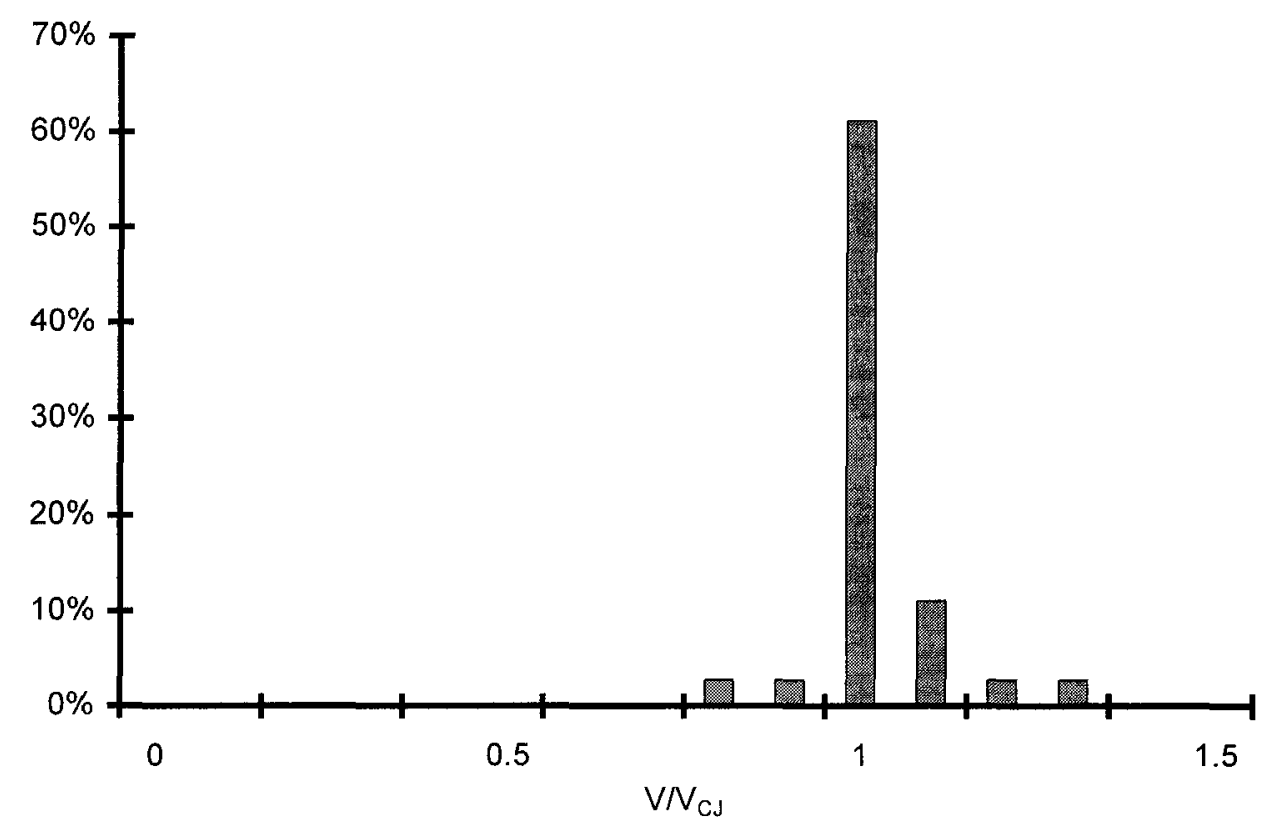

Fig. 15: a) Local velocity variation with distance along tube for ethylene-air near lean limit of quasi-detonation regime $(\phi=0.7, \mathrm{BR}=0.41)$.

b) Corresponding velocity histogram. 
a)

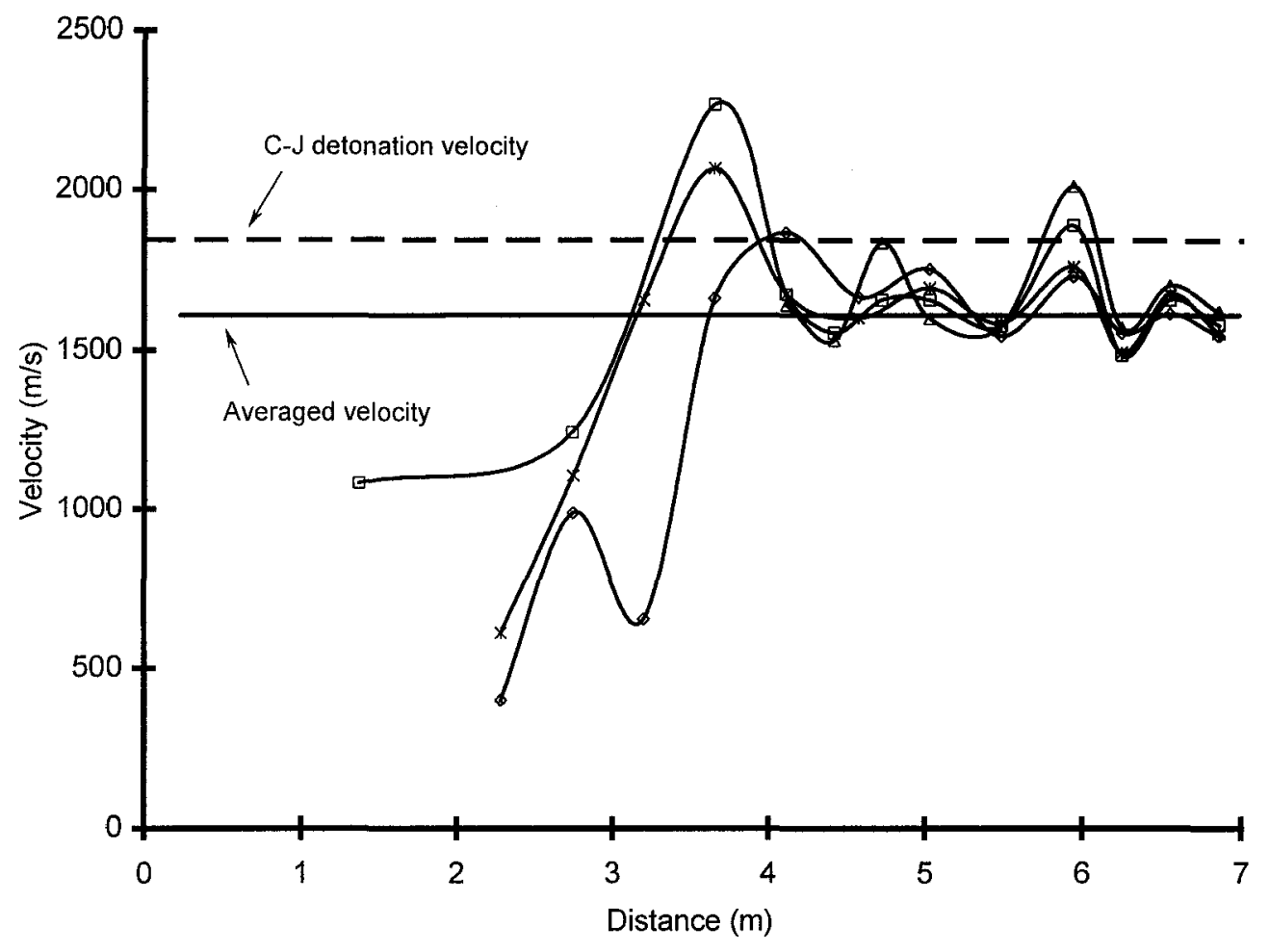

b)

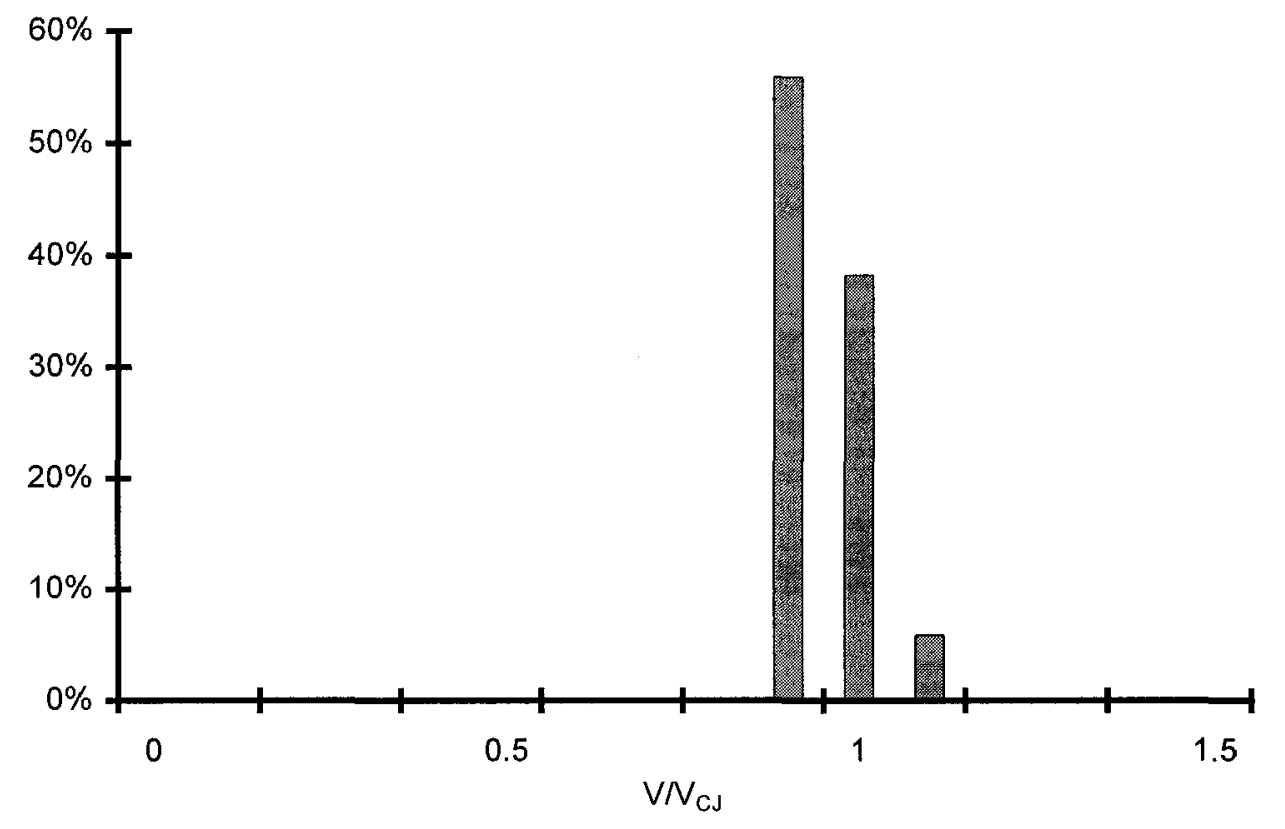

Fig. 16: a) Local velocity variation with distance along tube for ethylene-air near rich limit of quasi-detonation regime $(\phi=2.2, \mathrm{BR}=0.41)$.

b) Corresponding velocity histogram. 
a)

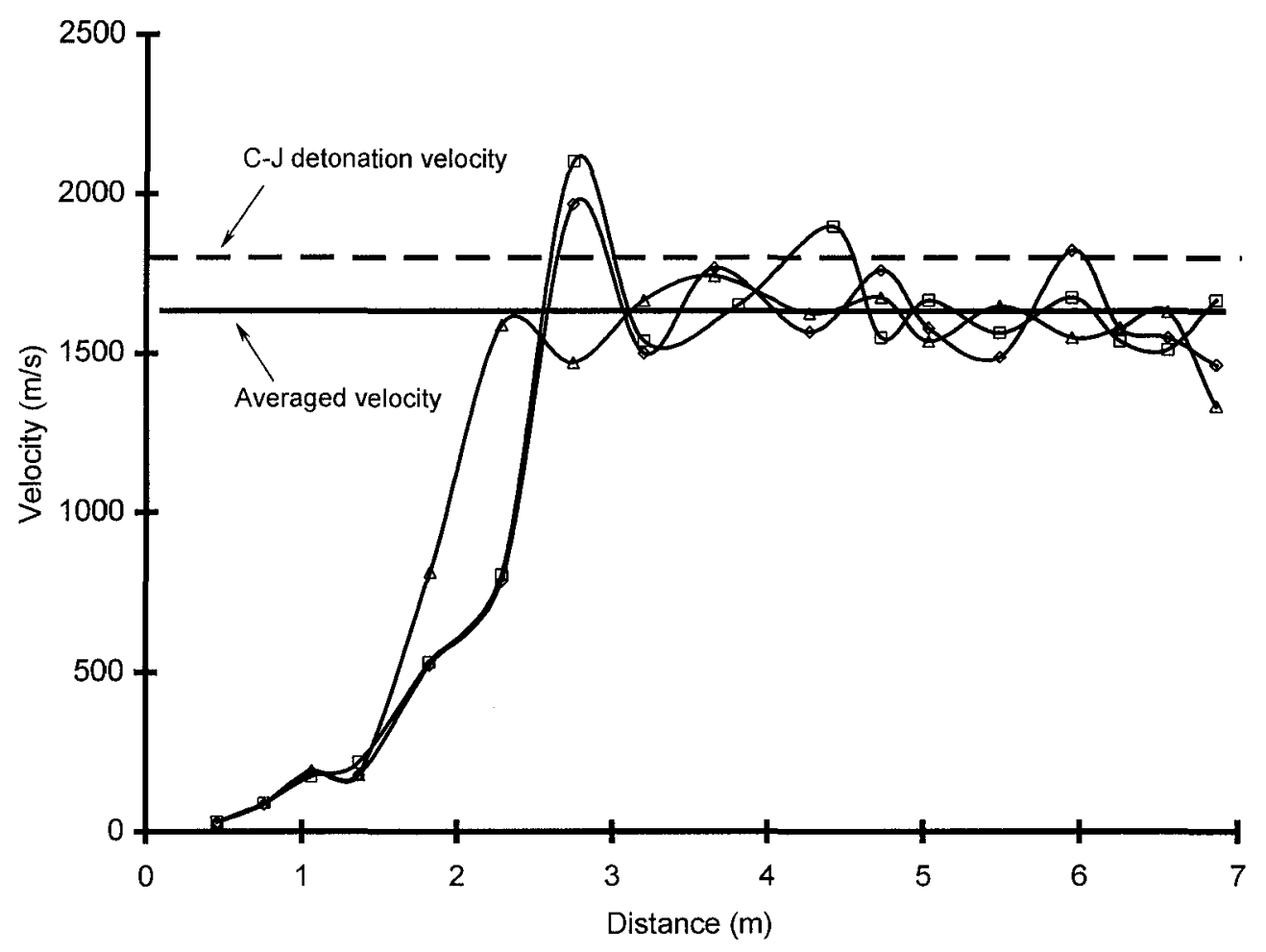

b)

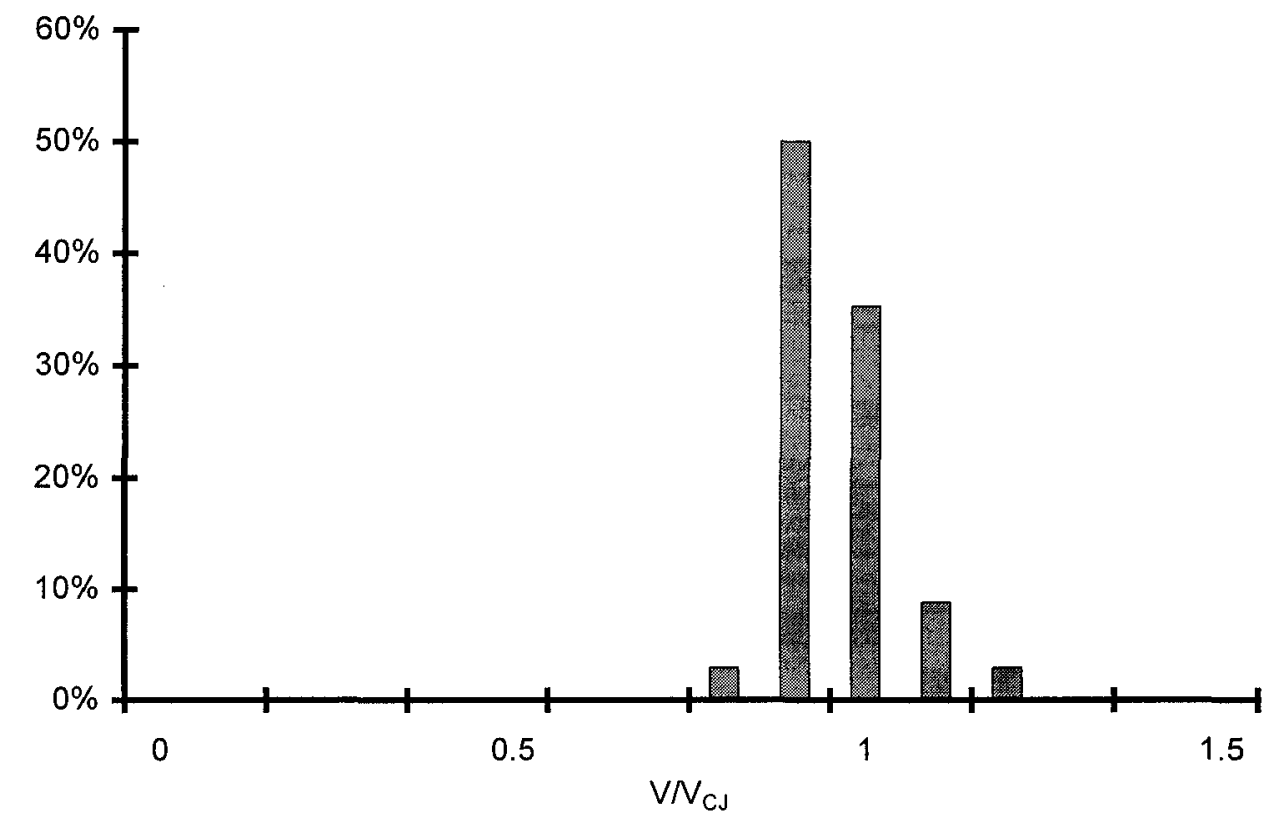

Fig. 17: a) Local velocity variation with distance along tube for stoichiometric propane-air $(\phi=1.0, B R=0.41)$. b) Corresponding velocity histogram. 
a)

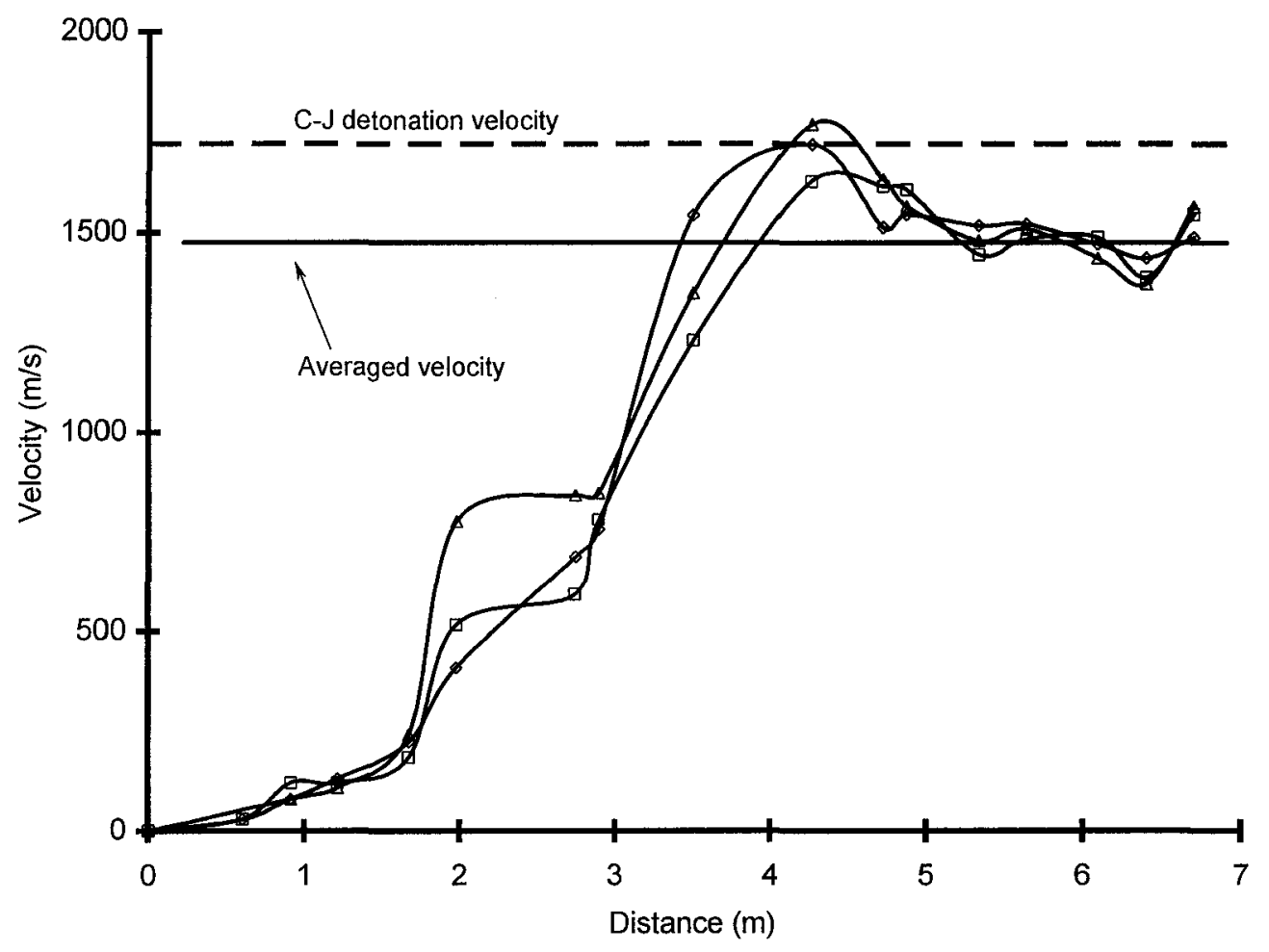

b)

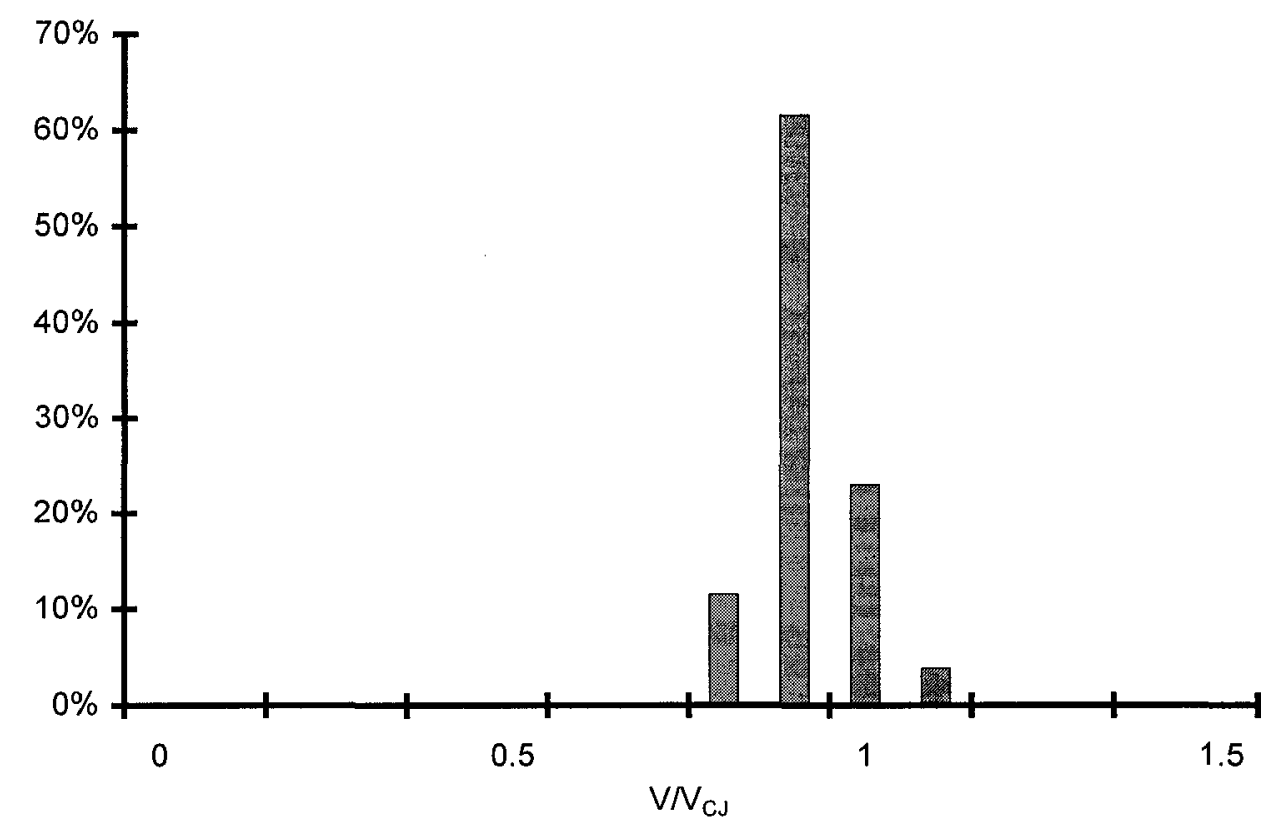

Fig. 18: a) Local velocity variation with distance along tube for propane-air near lean limit of quasi-detonation regime $(\phi=0.8, \mathrm{BR}=0.41)$.

b) Corresponding velocity histogram. 
a)

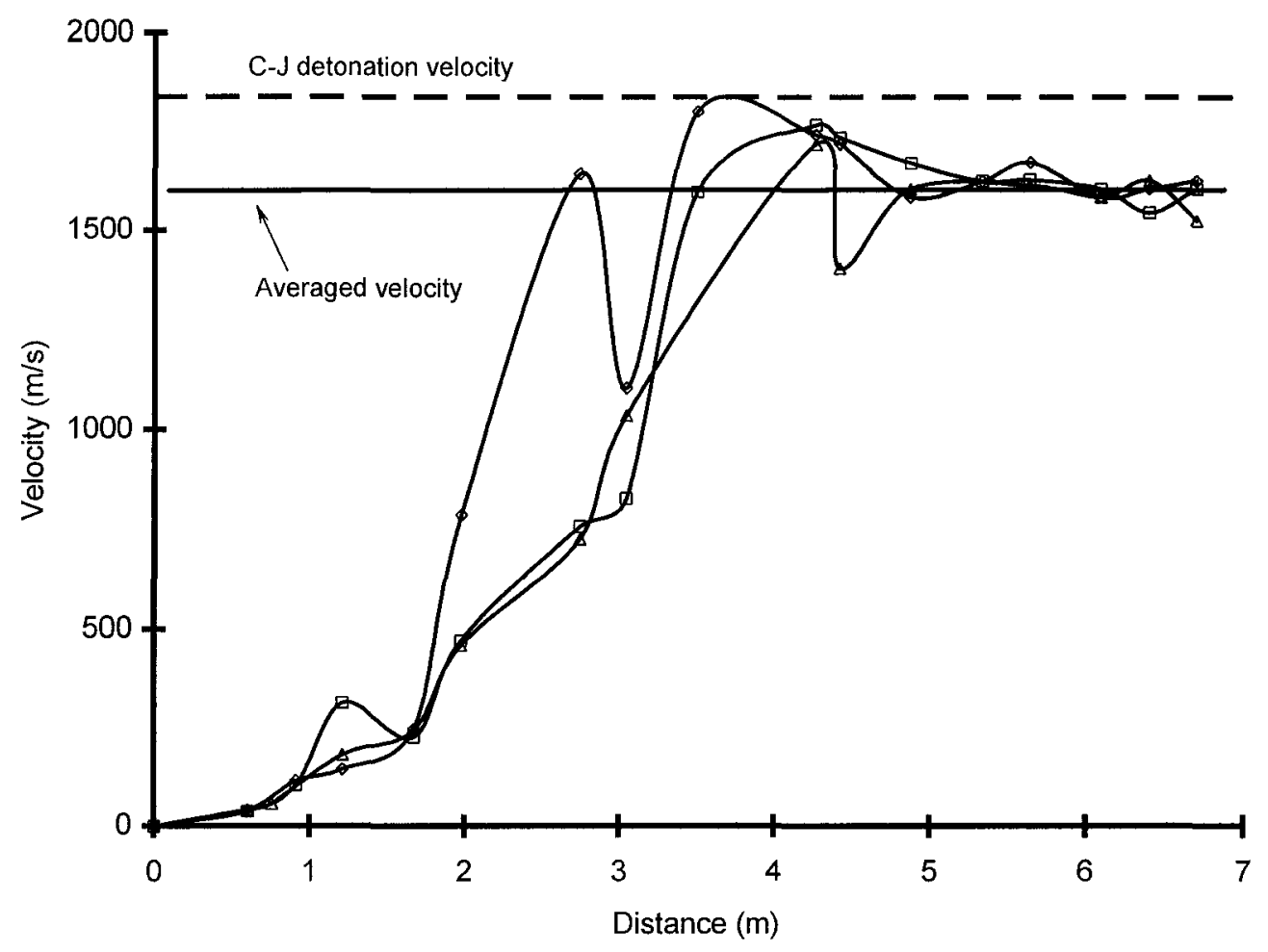

b)

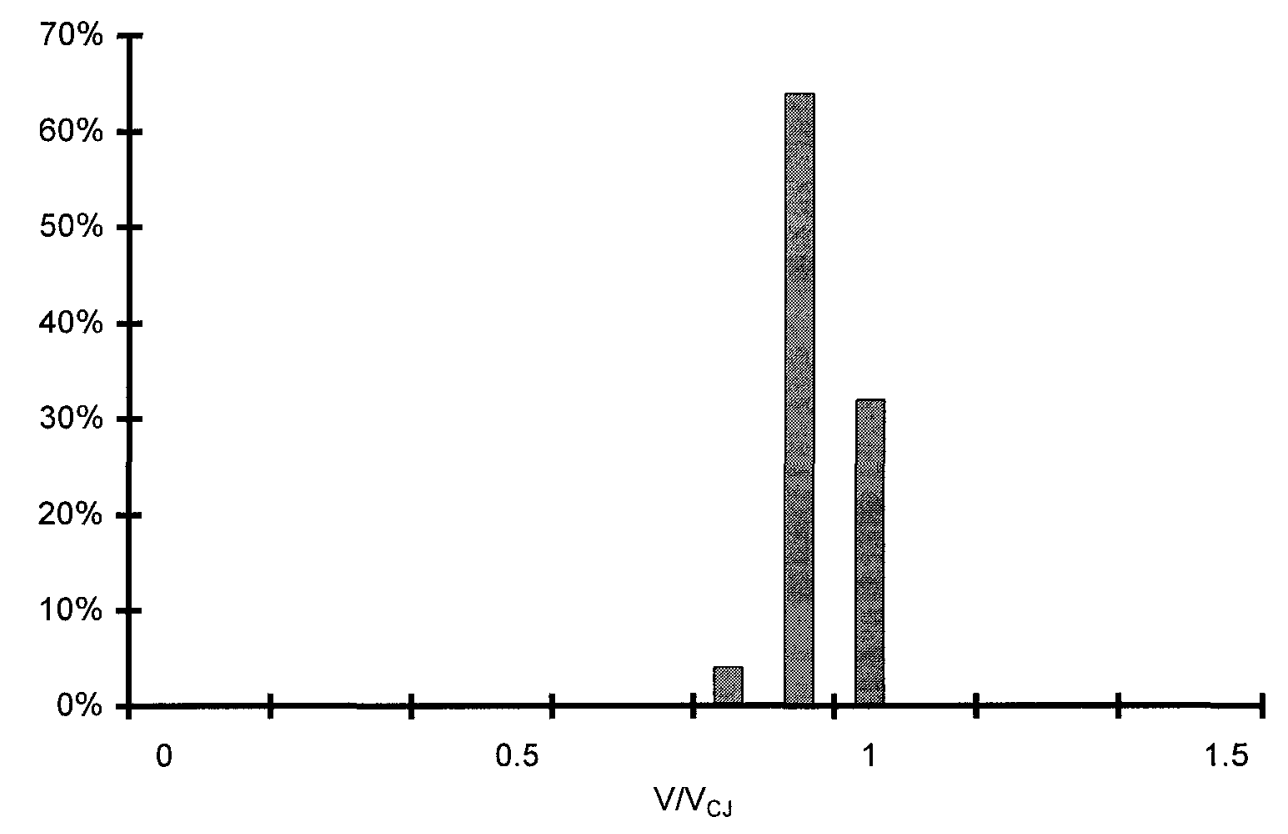

Fig. 19: a) Local velocity variation with distance along tube for propane-air near rich limit of quasi-detonation regime $(\phi=1.3, B R=0.41)$.

b) Corresponding velocity histogram. 


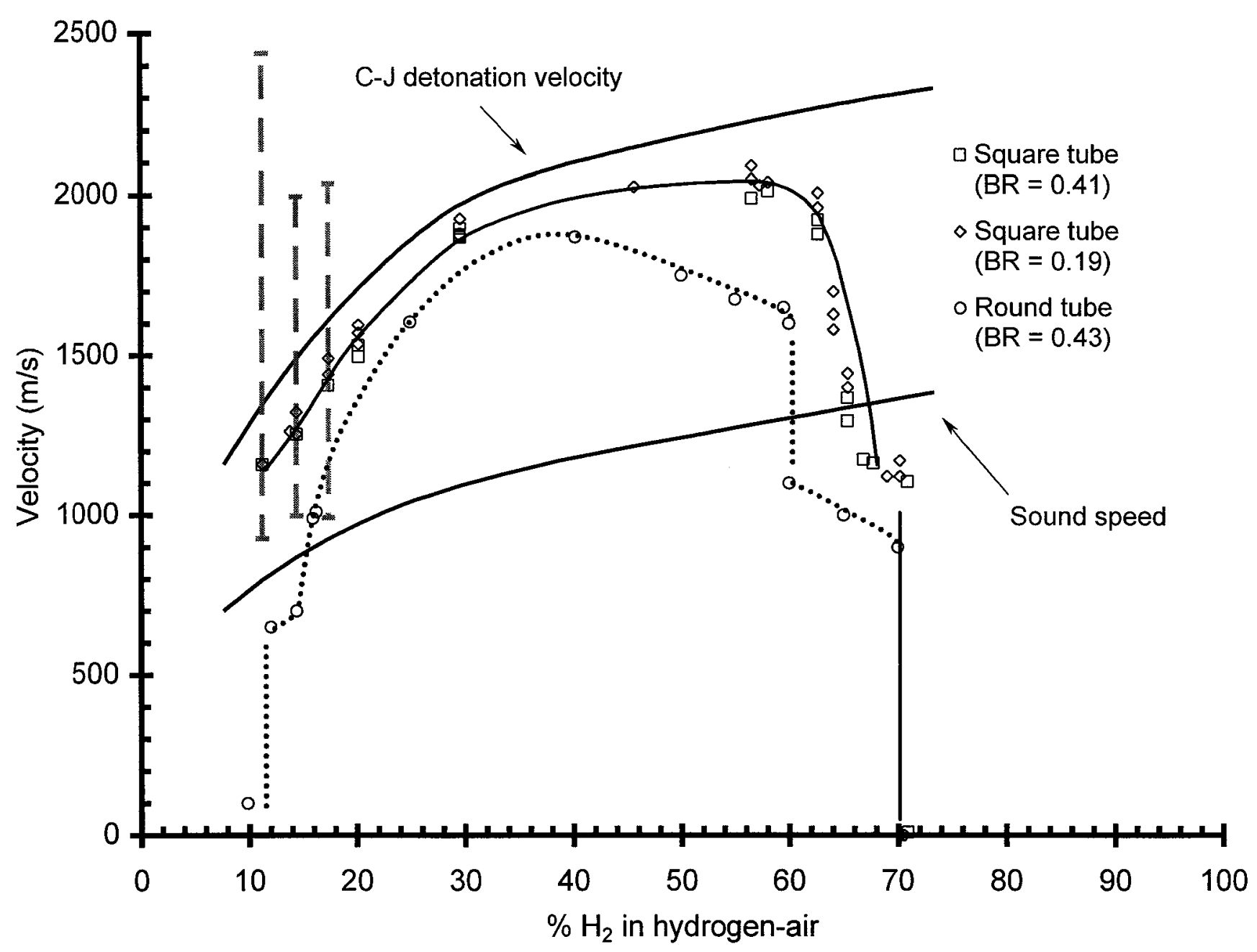

Fig. 20: Variation of velocity with \% hydrogen in hydrogen-air. 


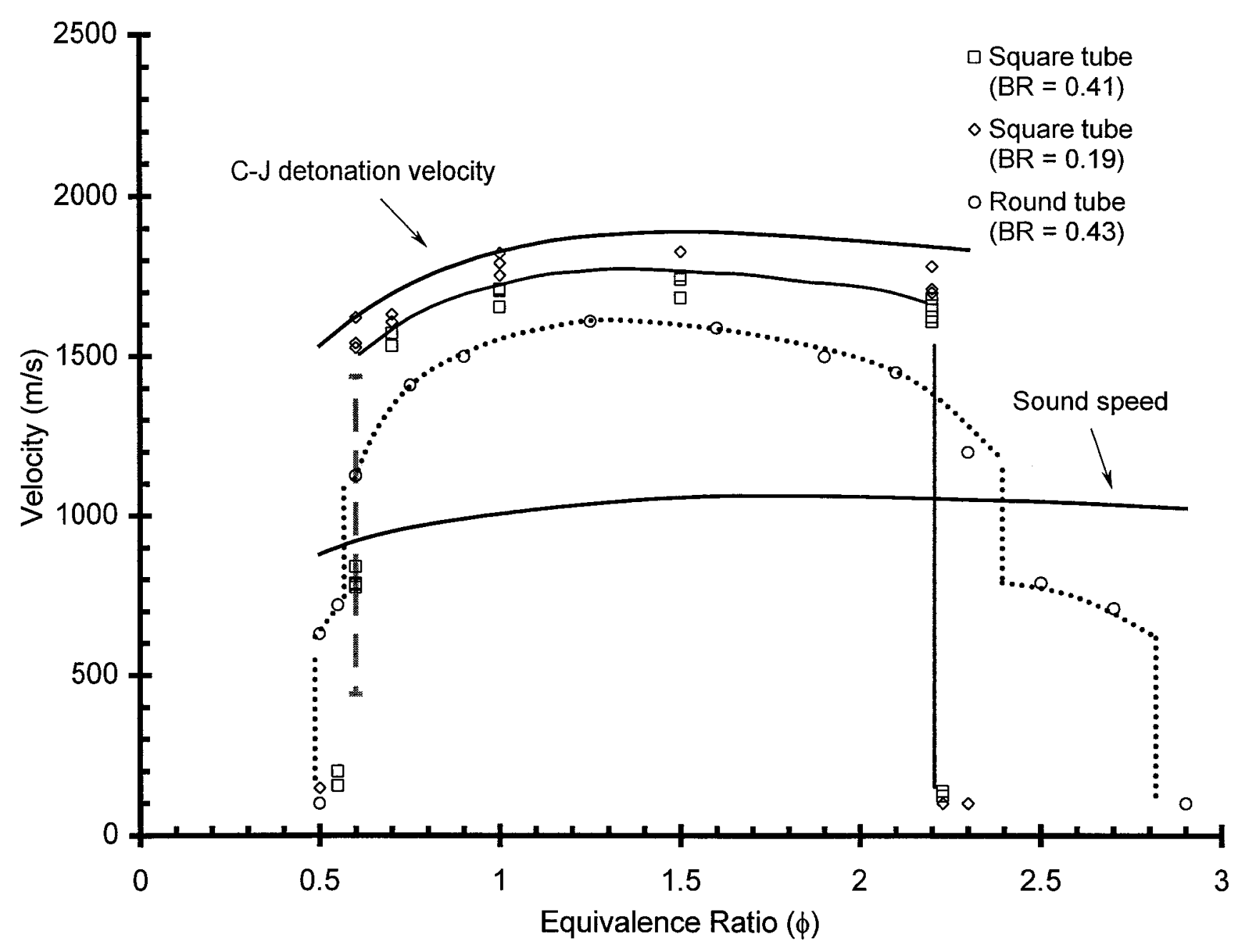

Fig. 21: Variation of velocity with equivalence ratio for ethylene-air. 


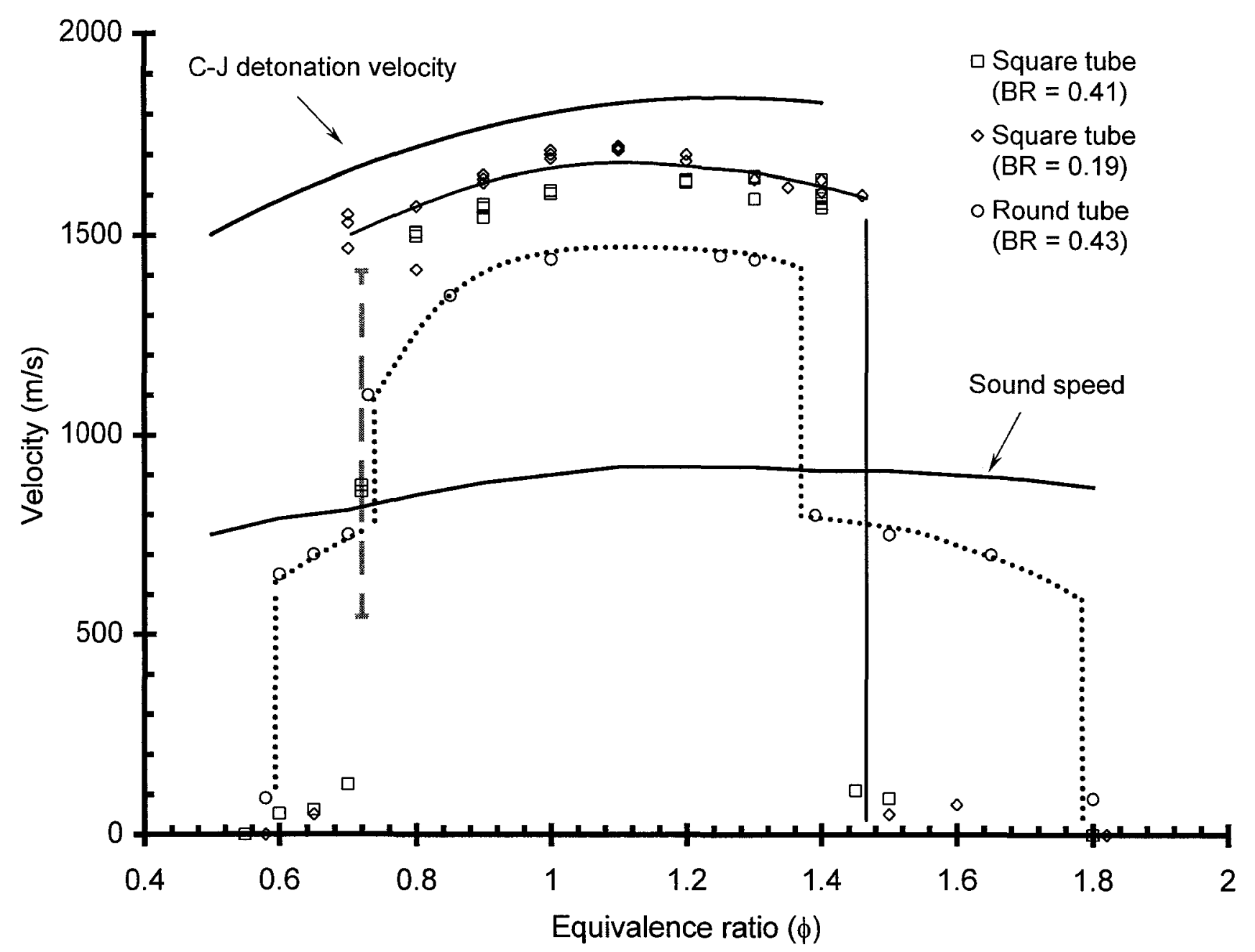

Fig. 22: Variation of velocity with equivalence ratio for propane-air. 


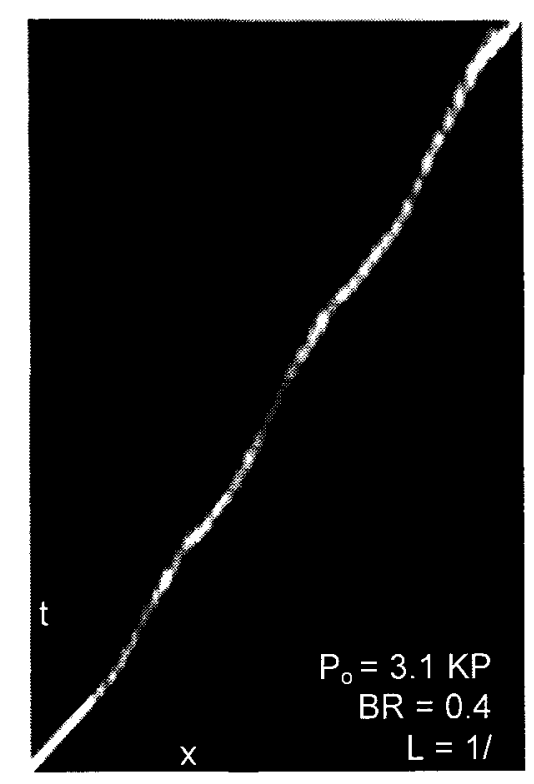

a)

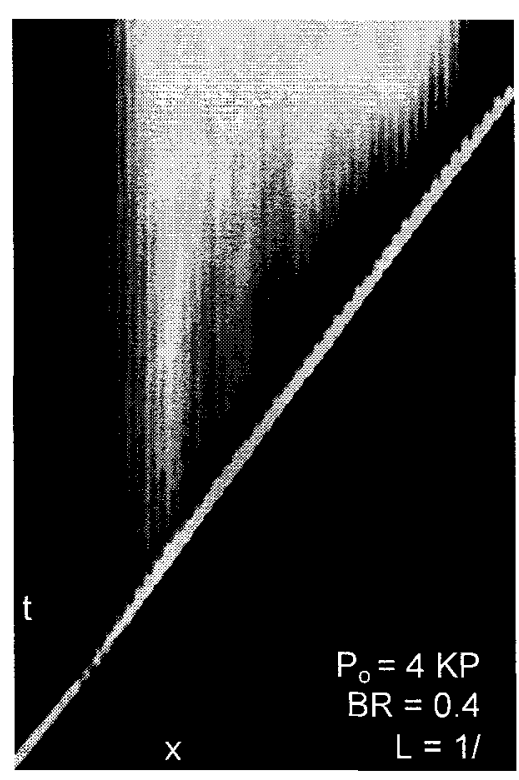

b)

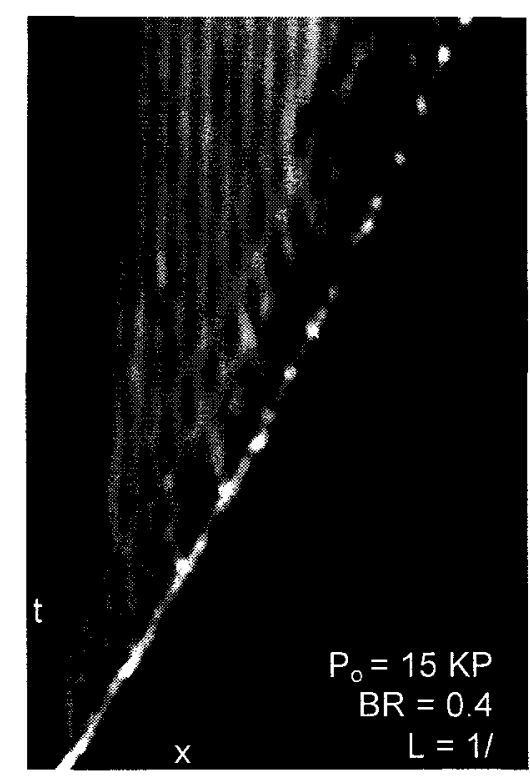

c)

Fig. 29: Streak photographs of a) unstable flame propagation at transition limit between quasi-detonation regime and “choking" regime, b) stable flame propagation in quasi-detonation regime, and c) stable flame propagation at transition limit with $\mathbf{7 5 \%}$ argon dilution (all in stoichiometric acetylene-oxygen). 


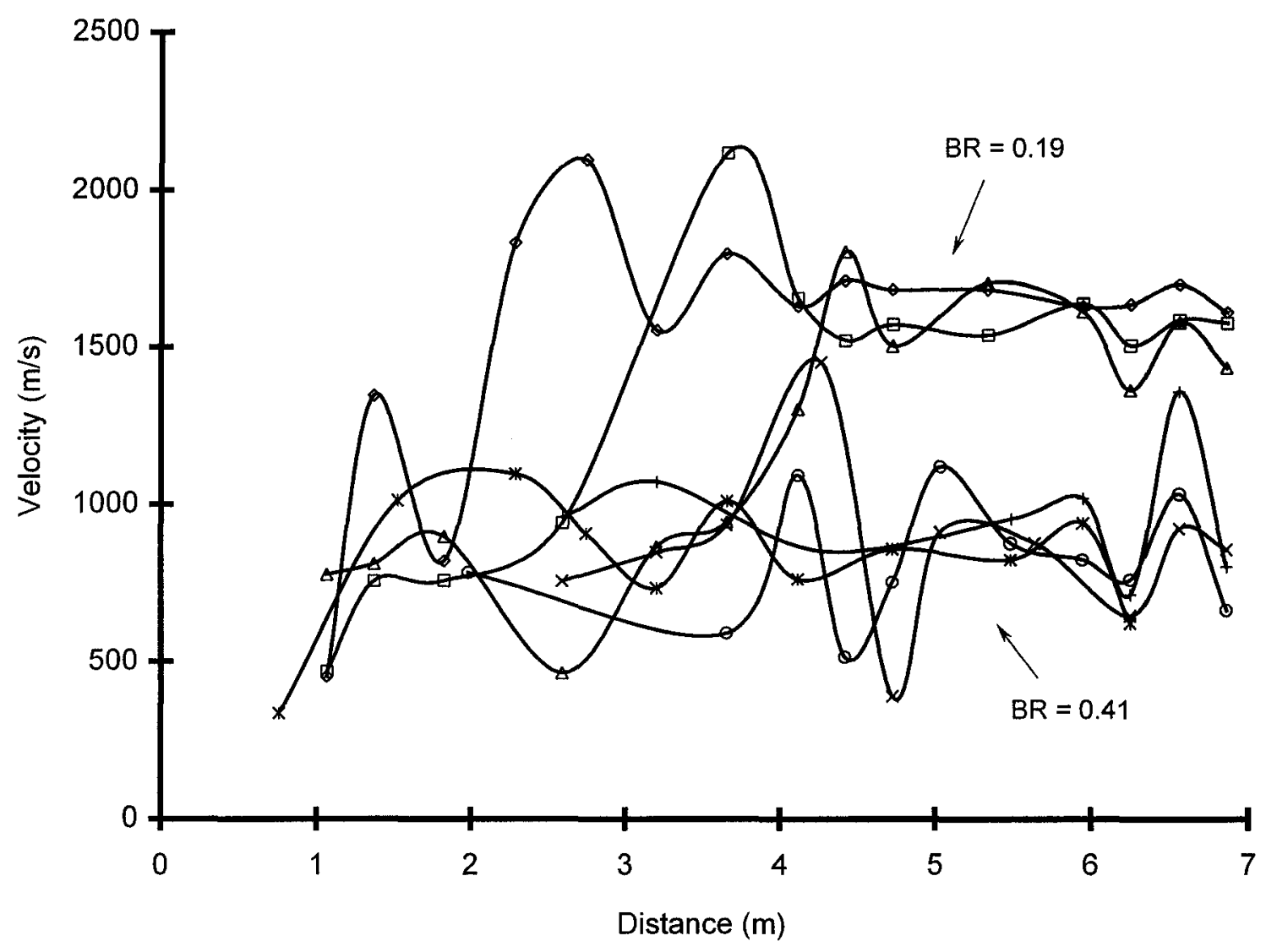

Fig. 31: Local velocity variation with distance along tube for lean propane-air $(\phi=0.7)$ using different blockage ratio at lean transition limit. 

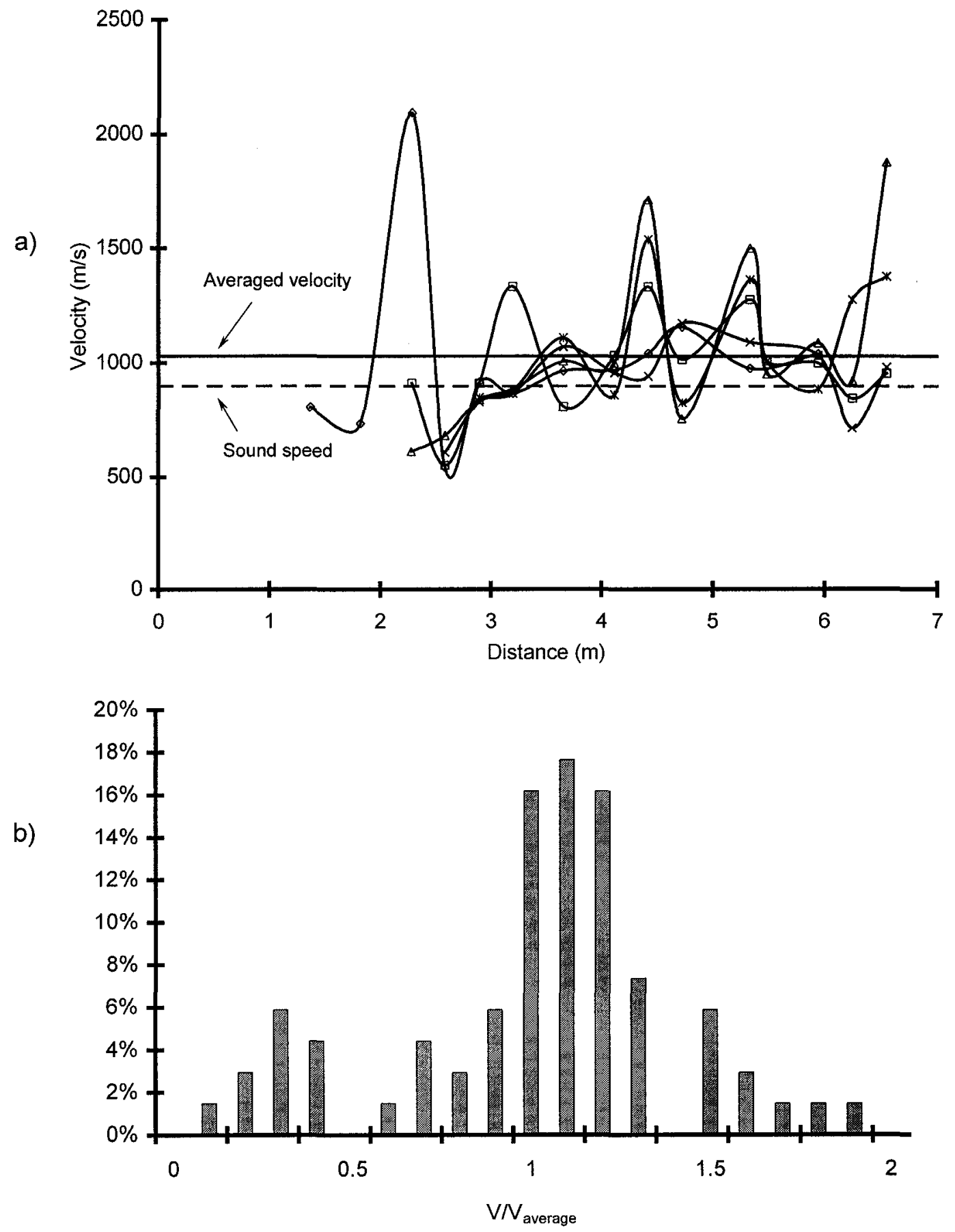

Fig. 32: a) Local velocity variation with distance along tube for stoichiometric methane-air $(\phi=1.0, B R=0.41)$. b) Corresponding velocity histogram. 


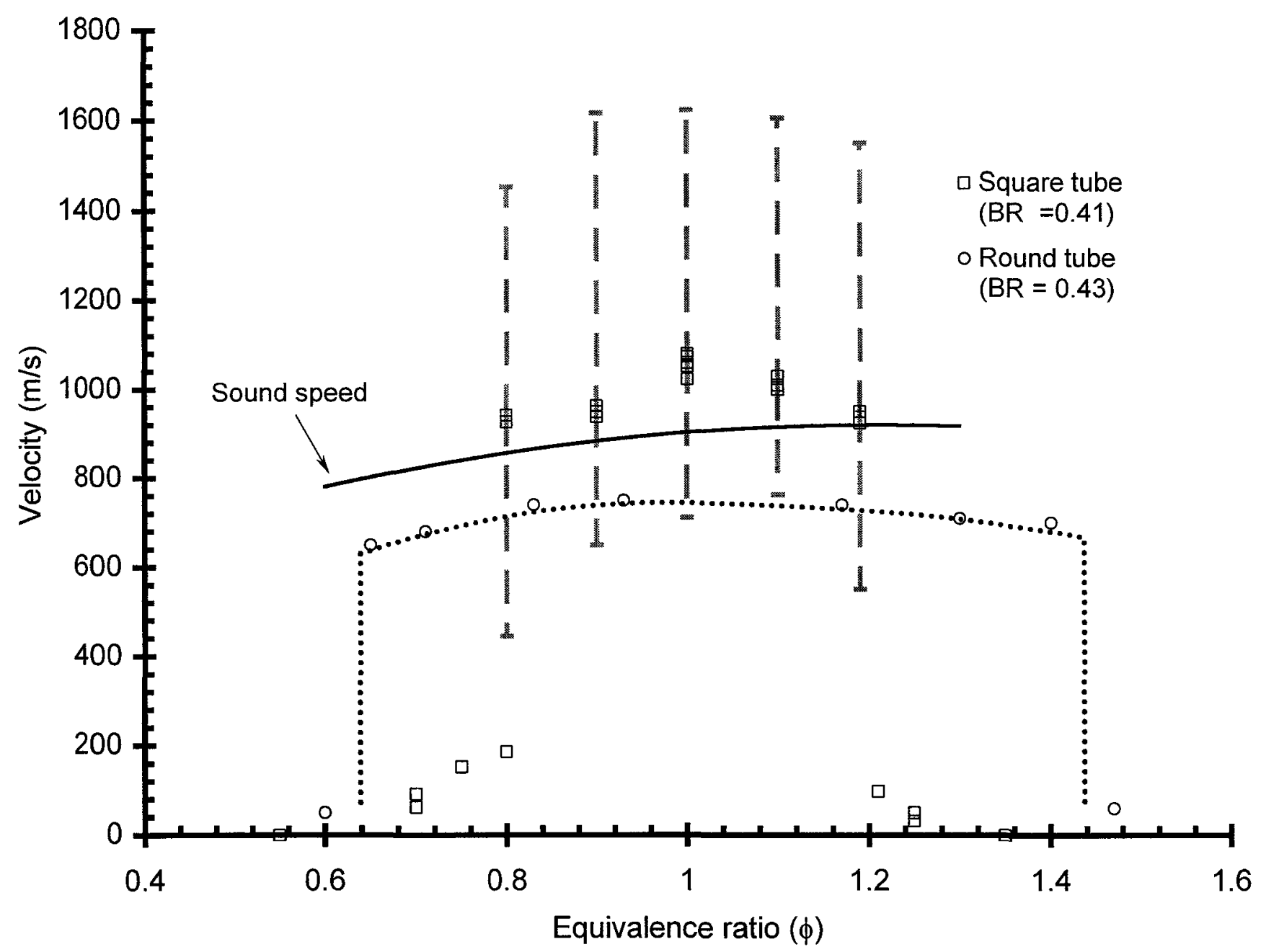

Fig. 33: Variation of velocity with equivalence ratio for methane-air. 


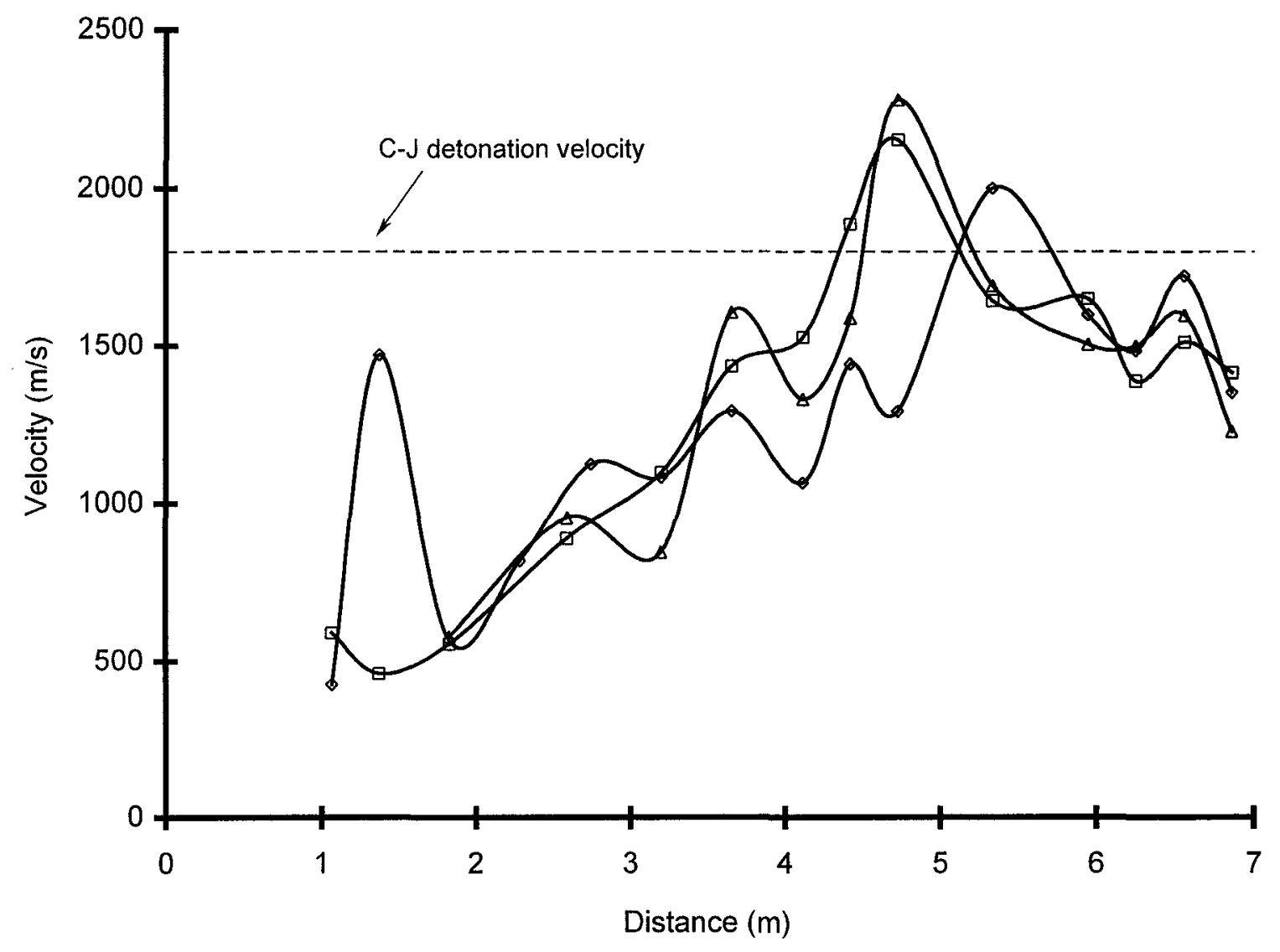

Fig. 34: Local velocity variation with distance along tube for stoichiometric methane-air $(\phi=1.0, B R=0.19)$. 


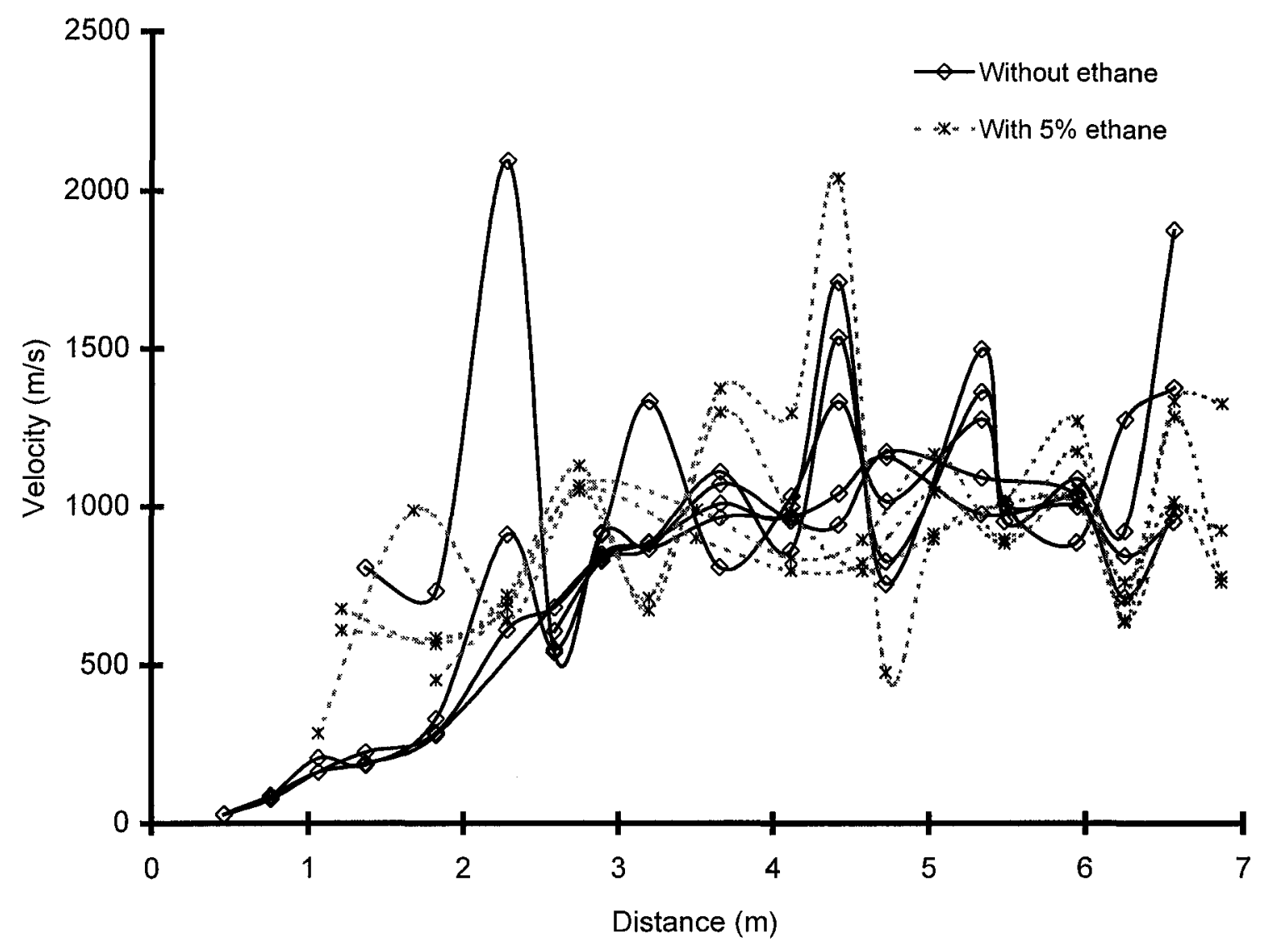

Fig. 35: Local velocity variation with distance along tube for stoichiometric methane-air with $5 \%$ ethane $(\phi=1.0, B R=0.41)$. 


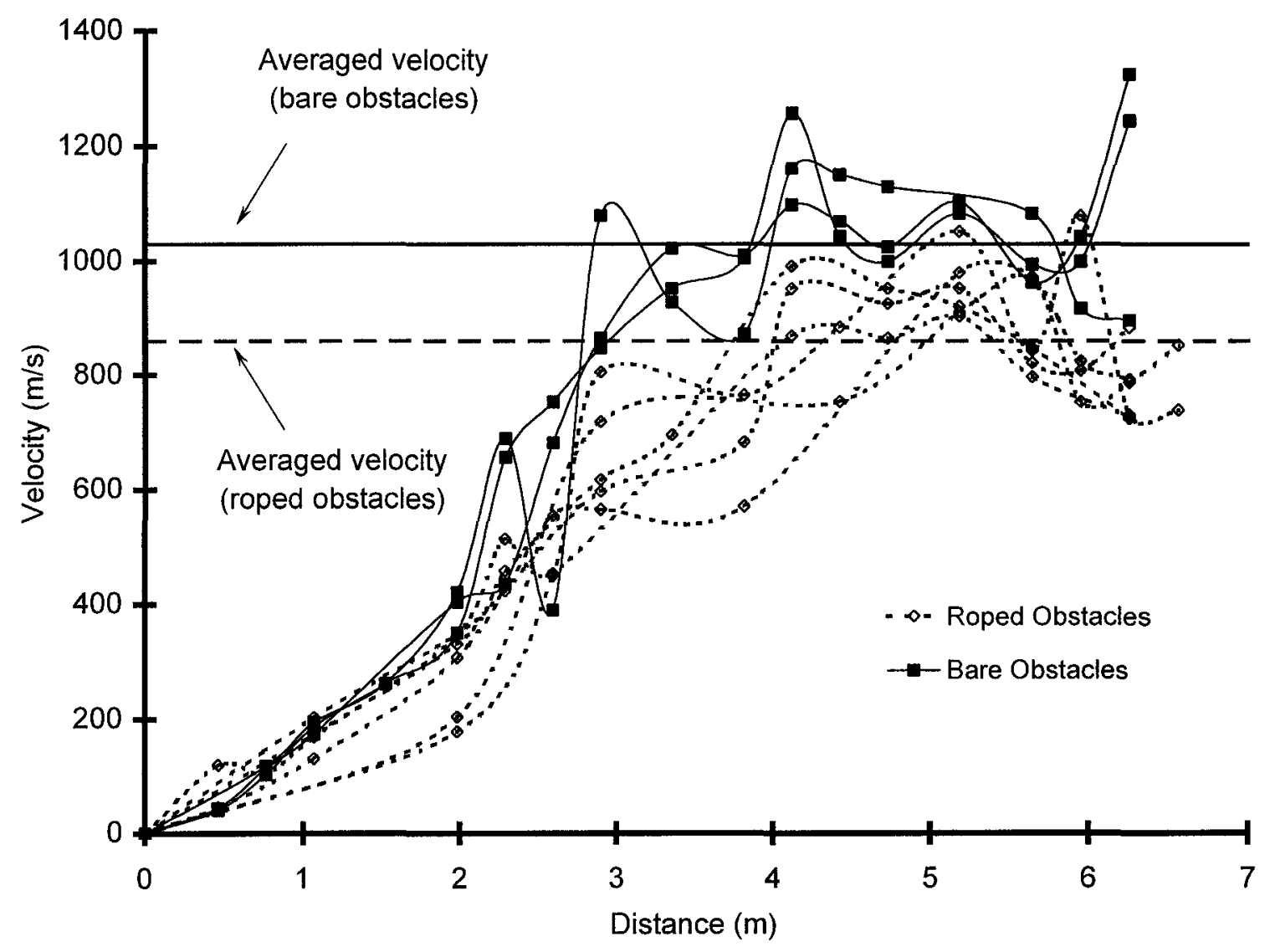

Fig. 36: Local velocity variation with distance along tube for stoichiometric methane-air $(\phi=1.0, B R=0.41)$ with and without acoustically absorbing material. 


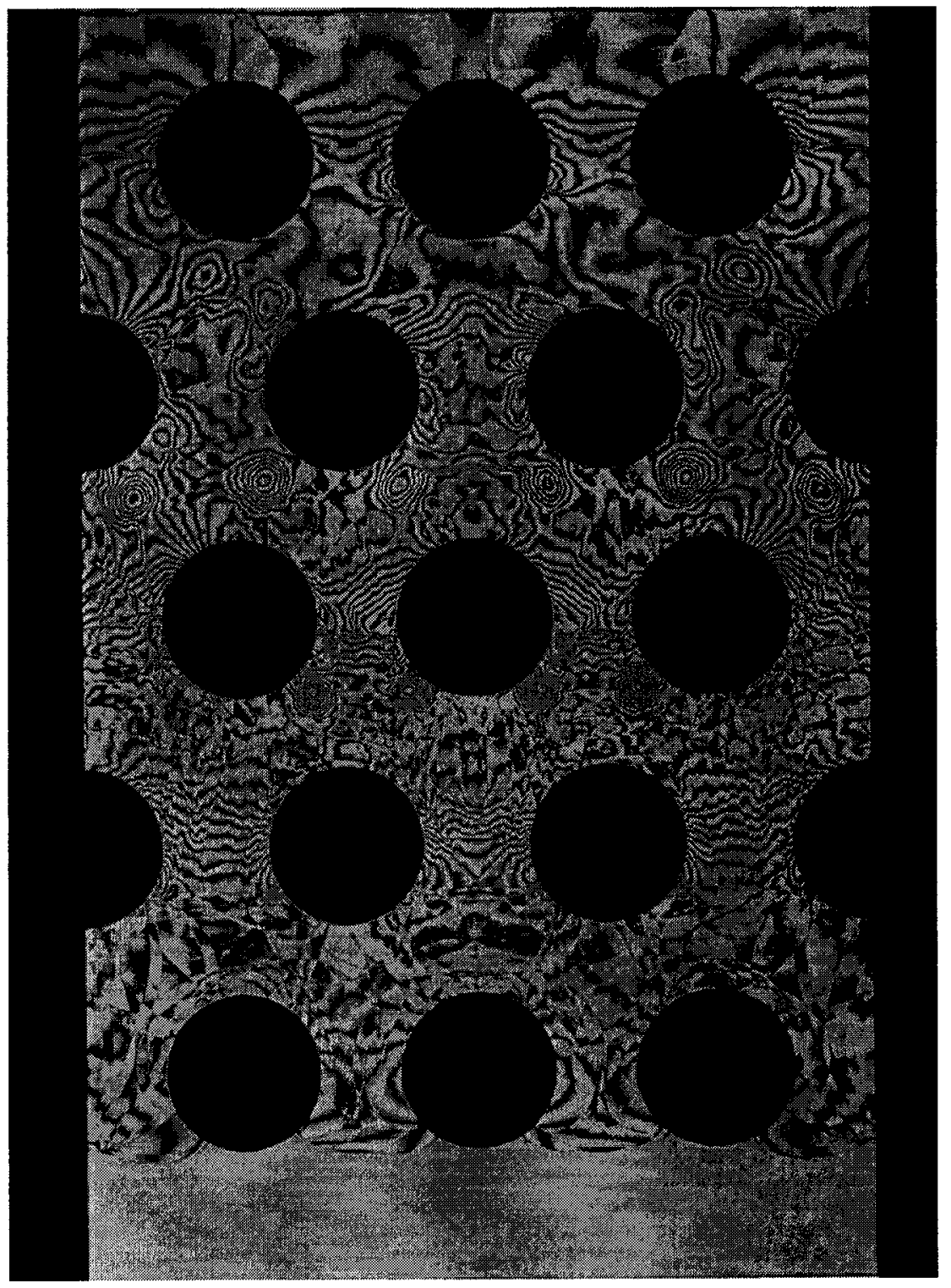

Fig. 37: Open shutter photograph of shock wave $\left(M_{s} \approx 1.3\right)$ propagating through dense obstacle field. 


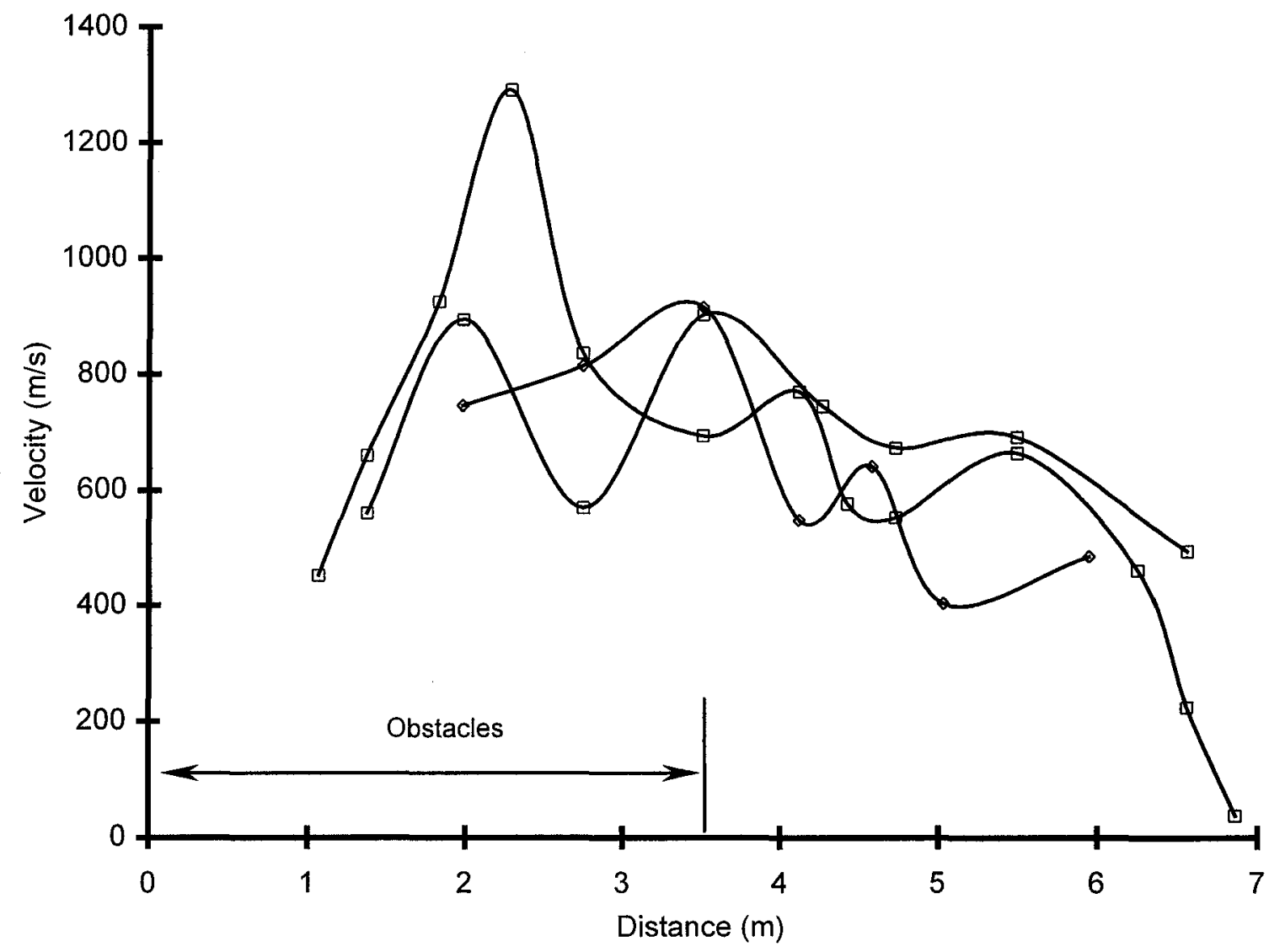

Fig. 38: Local velocity variation with distance along tube for stoichiometric methane-air $(\phi=1.0, B R=0.41)$ into an empty tube. 